\title{
Inclusive and exclusive electron scattering data analysis from Jefferson Lab experiment E12-14-012
}

\author{
Matthew Douglas Murphy \\ Dissertation submitted to the Faculty of the \\ Virginia Polytechnic Institute and State University \\ in partial fulfillment of the requirements for the degree of \\ Doctor of Philosophy \\ in \\ Physics \\ Camillo Mariani, Chair \\ Shunsaku Horiuchi \\ Patrick Huber \\ Leo Piilonen
}

December 16, 2020

Blacksburg, Virginia

Copyright 2021, Matthew Douglas Murphy 


\title{
Inclusive and exclusive electron scattering data analysis from Jefferson Lab experiment E12-14-012
}

\author{
Matthew Douglas Murphy
}

(ABSTRACT)

Since the first observations of neutrino oscillation, neutrino experiments have come a long way toward precise measurements of the neutrino oscillation parameters, but some obstacles still remain. The next generation of oscillation experiments, including the Deep Underground Neutrino Experiment (DUNE), will be using the Liquid Argon Time Projection Chambers (LArTPCs) with natural argon as the neutrino target material. A precise model of the neutrino cross section on argon does not exist, which is a source of significant uncertainty in such experiments. The E12-14-012 experiment at Jefferson Lab seeks to help remedy this via electron scattering measurements on argon and titanium targets. The experiment collected both inclusive (e,e $\left.e^{\prime}\right)$ and exclusive (e,e'p) data at a wide range of kinematics with the intent to measure the electron-nucleus cross section and thus derive a spectral function for argon that can be used with neutrino experiments. The use of titanium in this experiment stems from the equivalent shell structure that its protons share with the neutrons in argon, which will be crucial in oscillation experiments but cannot be measured directly in electron scattering. This thesis collects several papers which present results from the E12-14-012 experiment. These results include the inclusive $\left(\mathrm{e}, \mathrm{e}^{\prime}\right)$ cross sections for carbon, titanium, argon, and aluminum at a beam energy of $2.22 \mathrm{GeV}$ and a scattering angle of $15.54^{\circ}$ with uncertainty of less than $5 \%$. Also included are the first results of the exclusive (e,e'p) cross section on argon and titanium. 


\title{
Inclusive and exclusive electron scattering data analysis from Jefferson Lab experiment E12-14-012
}

\author{
Matthew Douglas Murphy
}

(GENERAL AUDIENCE ABSTRACT)

Since the first observations of neutrino oscillation, neutrino experiments have come a long way toward precise measurements of the neutrino oscillation parameters, but some of the properties of neutrinos still remain uncertain. The next generation of neutrino oscillation experiments, including the Deep Underground Neutrino Experiment (DUNE), will be using the Liquid Argon Time Projection Chambers (LArTPCs) with natural argon as the neutrino target material. A precise model of the neutrino cross section on argon does not exist, which will reduce the opportunity for DUNE to measure the neutrino properties with high precision. The E12-14-012 experiment at Jefferson Lab seeks to help remedy this via electron scattering measurements on argon and titanium targets. The experiment collected both inclusive $\left(e, e^{\prime}\right)$ and exclusive $\left(e, e^{\prime} p\right)$ data.The goal of this experiment is measure the electronnucleus interactions and provide a nuclear model for electron and neutrino interactions. This Ph.D. thesis collects several papers which present the results from the Jefferson lab E12-14012 experiment. 


\section{Acknowledgments}

First and foremost, I would like to thank my advisor, Professor Camillo Mariani, whose constant guidance has been indispensable and without whom none of this work would have been possible.

I would like to thank as well all the members of the argon collaboration for all the insights and discussions they provided to help keep me pointed in the right direction, primary among them Professors Omar Benhar and Donal Day, postdoctoral researchers Vishvas Pandey, Artur Ankowski, and Libo Jiang, and fellow graduate students Hongxia Dai, Linjie Gu, and Daniel Abrams.

Additionally, I thank the whole of the Hall A collaboration at Jefferson Lab for their knowledge and guidance on the essential technical details of the systems in place there.

I thank my committee, Profs. Shunsaku Horiuchi, Patrick Huber, and Leo Piilonen, for their insightful questions as I have presented this work to them over the years, and for upholding the academic integrity of the $\mathrm{PhD}$ process.

Lastly I thank my family and friends for all the emotional support they have provided over the years. 


\section{Contents}

List of Figures $\quad$ ix

List of Tables $\quad$ xvii

1 Introduction and Background 1

1.1 Neutrino Oscillation ......................... 1

1.1.1 Two flavor oscillations . . . . . . . . . . . . . . 1

1.1.2 Three flavor oscillations ..................... 3

1.2 Neutrino oscillation experiments . . . . . . . . . . . . . . 5

1.2.1 Neutrino-nucleus cross section . . . . . . . . . . . . . . 6

1.2.2 Neutrino Energy Reconstruction . . . . . . . . . . . . . . . 9

1.2.3 Past Experimental Oscillation Results . . . . . . . . . . . . . 12

1.2.4 Deep Underground Neutrino Experiment - DUNE . . . . . . . . . . 13

1.3 Electron scattering . . . . . . . . . . . . . . . . . . . 17

1.3.1 Inclusive cross section $\left(\mathrm{e}, \mathrm{e}^{\prime}\right) \ldots \ldots \ldots \ldots$

1.3.2 Exclusive cross section $\left(\mathrm{e}, \mathrm{e}^{\prime} \mathrm{p}\right) \ldots \ldots \ldots . \ldots . \ldots . \ldots 21$

1.3.3 PWIA - Plane Wave Impulse Approximation . . . . . . . . . . . . . . 24

1.3.4 DWIA - Distorted Wave Impulse Approximation . . . . . . . . . . . 27 
2 Experimental Details

2.1 Hall A Overview . . . . . . . . . . . . . . . . . . . 29

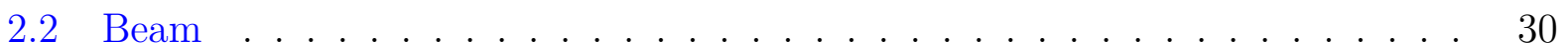

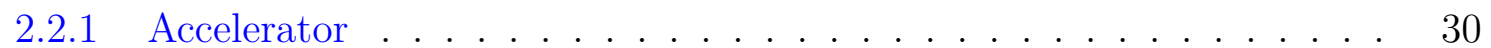

2.2.2 Beam Position Measurement . . . . . . . . . . . . . . . . 30

2.2.3 Beam Current Measurement . . . . . . . . . . . . . . . 32

2.2.4 Beam Energy Measurement . . . . . . . . . . . . . . . 32

2.3 HRS - High Resolution Spectrometers . . . . . . . . . . . . . . 33

2.4 Detector Package . . . . . . . . . . . . . . . . . . . . 34

2.4.1 Vertical Drift Chambers ................. 34

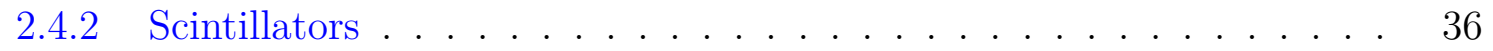

2.4.3 Gas Čerenkov detector . . . . . . . . . . . . . . 36

2.4.4 Lead Glass Calorimeters . . . . . . . . . . . . . . . . 37

2.5 Hall A E12-14-012 target system . . . . . . . . . . . . . . . . . . . . 38

2.6 Triggers and Data Acquisition . . . . . . . . . . . . . . . . . . . . 40

2.7 Monte Carlo . . . . . . . . . . . . . . . . . . . . . . . . 41

2.7.1 Radiative Corrections . . . . . . . . . . . . . . . . . 44

2.7.2 Coulomb Correction ................... . . 45

2.7.3 Energy Loss . . . . . . . . . . . . . . . . . . . . 45

2.7.4 Target model . . . . . . . . . . . . . . . . 46 
2.8 Data Collection . . . . . . . . . . . . . . . . . . . . . . . . 47

$3 \quad$ First Measurement of the Ti $\left(e, e^{\prime}\right) X$ Cross Section at Jefferson Lab 48

3.1 Introduction . . . . . . . . . . . . . . . . . . . . . . . . 48

3.2 Experimental Setup . . . . . . . . . . . . . . . . . . . . . . 51

3.3 Data Analysis . . . . . . . . . . . . . . . . . . . . . . . . . . . . . 52

3.4 Experimental Results and Discussion . . . . . . . . . . . . . . . 53

3.5 Summary and Conclusions . . . . . . . . . . . . . . . . . 58

4 First Measurement of the $\operatorname{Ar}\left(e, e^{\prime}\right) X$ Cross Section at Jefferson Lab 61

4.1 Introduction . . . . . . . . . . . . . . . . . . 61

$4.2 \quad$ Experimental Setup . . . . . . . . . . . . . . . . . . . . . . . . . 64

4.3 Data Analysis . . . . . . . . . . . . . . . . . . . . . . . . 65

4.4 Experimental Results and Scaling Analysis . . . . . . . . . . . . . . . 67

4.5 Summary . . . . . . . . . . . . . . . . . . . . . . . . . . 73

5 Measurement of the cross sections for inclusive electron scattering in the E12-14-012 experiment at Jefferson Lab $\quad 74$

5.1 Introduction . . . . . . . . . . . . . . . . . . . . . . 75

5.2 Experimental Setup . . . . . . . . . . . . . . . . . . . . . . . . 78

5.3 Data Analysis . . . . . . . . . . . . . . . . . . . . . . . . . . . . . . . 82

5.3 .1 Yield-Ratio Method . . . . . . . . . . . . . . . . . . . . . 82 
5.3.2 Acceptance Method . . . . . . . . . . . . . . . . 82

5.4 Uncertainty Analysis . . . . . . . . . . . . . . . . . . . . . . 88

5.5 Experimental Results . . . . . . . . . . . . . . . . . . . . . 91

5.6 Scaling and $A$-dependence . . . . . . . . . . . . . . . . . . . . . . . . . 97

5.7 Summary and Conclusions . . . . . . . . . . . . . . . . . . . . . 101

6 Measurement of the $\operatorname{Ar}\left(e, e^{\prime} p\right) X$ and $\operatorname{Ti}\left(e, e^{\prime} p\right) X$ cross sections in Jefferson

$\begin{array}{ll}\text { Lab Hall A } & 103\end{array}$

6.1 Introduction . . . . . . . . . . . . . . . . . . . . 103

6.2 Experimental Setup . . . . . . . . . . . . . . . . . . . . 108

6.3 Data Analysis . . . . . . . . . . . . . . . . . . . . . . . . . 112

6.3.1 The $\left(e, e^{\prime} p\right)$ cross section . . . . . . . . . . . . . 112

6.3.2 Data analysis details ................... 116

6.3.3 Test spectral functions . . . . . . . . . . . . . . . 118

6.4 Uncertainty Analysis . . . . . . . . . . . . . . . . . . . . . . . 122

6.4.1 Final state interactions . . . . . . . . . . . . . . . 126

6.5 Differential cross section comparison . . . . . . . . . . . . . . . 131

6.6 Summary and Conclusions . . . . . . . . . . . . . . . . . 135

$\begin{array}{lll}7 & \text { Summary and Conclusions } & 137\end{array}$

$\begin{array}{ll}\text { Bibliography } & 138\end{array}$ 


\section{List of Figures}

1.1 Reconstructed neutrino energy distributions using the FG model and SF model. The plot on the top shows the results at $E_{\mu}=600 \mathrm{MeV}$ and $\theta_{\mu}=60^{\circ}$, on the bottom is at $E_{\mu}=1 \mathrm{GeV}$ and $\theta_{\mu}=35^{\circ}[5] \ldots \ldots 11$

1.2 $1 \sigma$ confidence regions of $\left(\theta_{13}, \delta_{C P}\right)$ using data from simulation. The results are shown when the migration matrices accounting for $90 \%, 80 \%$, and $70 \%$ of the missing energy, while the green area shows the case for all of the missing energy. The true values of $\theta_{13}$ and $\delta_{C P}$ are shown as the black dot in the center of the green area $[40] \ldots \ldots \ldots \ldots \ldots \ldots$

1.3 Schematic of the normal and inverted hierarchy definition [7]. In the plot the different colors correspond to the flavor contents for the corresponding neutrino mass eigenstates. . . . . . . . . . . . . . .

1.4 Schematic of the Deep Underground Neutrino Experiment [10]. . . . . . . .

1.5 Top-left plot represents the neutrino oscillation probability $P\left(\nu_{\mu} \rightarrow \nu_{e}\right)$ as a function of $L$ and $E_{\nu}$ with a value for $\delta_{C P}=0$ and assume NO for the mass hierarchy. The top right plot shows $P\left(\nu_{\nu} \rightarrow \nu_{e}\right)$ as a function of $E_{\nu}$ at the DUNE baseline (800 miles) for $\delta_{C P}=0,-\pi / 2$, and $\pi / 2$. The bottom plots show the same but for antineutrinos $\bar{\nu}_{\mu} \rightarrow \bar{\nu}_{e}[10] \ldots \ldots \ldots \ldots$

1.6 Same as Fig. 1.5 but assuming IO for the mass hierarchy $[10] . \ldots \ldots$. . . . 17

1.7 General $\left(\mathrm{e}, \mathrm{e}^{\prime}\right)$ energy spectrum $\ldots \ldots \ldots \ldots \ldots \ldots$

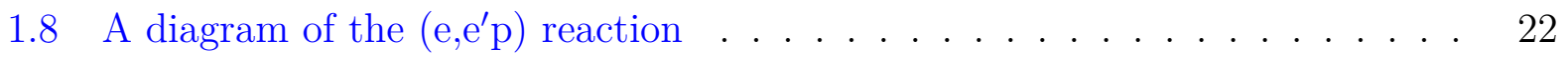


1.9 A diagram of the PWIA in $\left(\mathrm{e}, \mathrm{e}^{\prime} \mathrm{p}\right)$ reaction $[13] \ldots \ldots \ldots \ldots$

1.10 A diagram of the DWIA in $(\mathrm{e}, \mathrm{e} \mathrm{p})$ reaction $[13] \ldots \ldots \ldots 27$

2.1 Schematic of the accelerator facility, CEBAF. The beam is produced at the injector, and two superconducting linacs are designed to accelerate it up to 4 $\mathrm{GeV}$ before it is sent to Halls A, B, and C. . . . . . . . . . . . 31

2.2 Schematic of the beam position monitor system [16] . . . . . . . . . . 31

2.3 Schematic of the $e P$ energy measurement device [15] . . . . . . . . . . 34

2.4 Schematic of the HRS Vertical Drift Chambers [15] . . . . . . . . . . 35

2.5 Schematic of the mirrors and PMTs inside the HRS Gas Čerenkov detector. [15]. 37

2.6 Representation of the lead glass calorimeters in the left (top) and right (bottom) HRS $[15] . \ldots \ldots \ldots \ldots$

2.7 Photo showing the closed gas cell target (top), the aluminum dummy target (center), and the multi-foil carbon optical target (bottom) $[20] . \ldots \ldots$.

2.8 T1 efficiency as function of run number (red data points) and product of the T3 and T4 efficiency as function of run number.The red and green line are a fit with a polynomial of order zero to the data. . . . . . . . . . .

2.9 T1 and product T3*T4 efficiencies as function of the target length for the argon cell. . . . . . . . . . . . . . . . . . . . . 42

2.10 The incoming and/or scattered electron can lose energy through bremsstrahlung. 44

2.11 Schematic of our argon gas target cell. . . . . . . . . . . . . . . 46 
3.1 Comparison between the scaling function $F(y)$ obtained from the E12-14-012 data on $\mathrm{C}$, represented by diamonds, and those obtained from the data of O'Connell et al. [74], Sealock et al. [75], and Day et al. [76]. The inset shows the momentum transfer dependence of $F(y)$ at fixed $y=-0.2 \mathrm{GeV}$. The data sets are labeled by the value of $Q^{2}$ corresponding to the top of the quasielastic

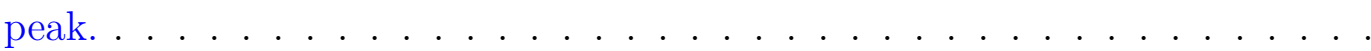

3.2 Double differential cross section for the $\mathrm{C}\left(e, e^{\prime}\right)$ process measured at beam energy of $2.222 \mathrm{GeV}$ and scattering angle of $15.541 \mathrm{deg}$. The inner and outer uncertainty bars correspond to statistical and total uncertainties, respectively. The solid line represents the theoretical results obtained within the formalism described in Refs. $[50,52,53,81] \ldots \ldots \ldots \ldots$

3.3 Double differential cross section for the $\operatorname{Ti}\left(e, e^{\prime}\right)$ process measured at beam energy of $2.222 \mathrm{GeV}$ and fixed scattering angle of $15.541 \mathrm{deg}$. The inner and outer uncertainty bars correspond to statistical and total uncertainties, respectively. The maximum uncertainties in the full kinematical range are

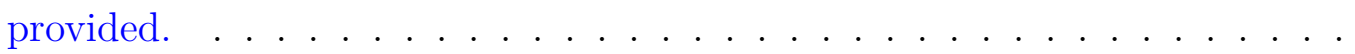

3.4 Ratios defined by Eq.(3.3), computed using the measured carbon and titanium cross sections. . . . . . . . . . . . . . . . . .

3.5 Comparison between the scaling function of $2^{\text {nd }}$ kind, $f(\psi)$, obtained from the E12-14-012 data on $\mathrm{C}$ and $\mathrm{Ti}$, represented by diamonds and circles, respsctively. The Fermi momentum of carbon has been fixed to the value obtained by Moniz et al. [84]. The data analysis for Ti sets the Ti Fermi momentum at $\sim 240 \mathrm{MeV} . \ldots \ldots \ldots \ldots \ldots \ldots \ldots \ldots$ 
4.1 Double differential cross section for the $\operatorname{Ar}\left(e, e^{\prime}\right)$ process, extracted with two different methods, at beam energy of $2.222 \mathrm{GeV}$ and scattering angle of $15.541 \mathrm{deg}$. The inner and outer bars correspond to the statistical and total uncertainty, respectively. The dotted curve represents the quasielastic calculations obtained within the relativistic Green's function (RGF) formalism described in Ref. [94]. . . . . . . . . . . . . . . . . .

4.2 Comparison of $\operatorname{Ar}\left(e, e^{\prime}\right)$ cross section of Fig. 4.1, and $\operatorname{Ti}\left(e, e^{\prime}\right)$ and $\mathrm{C}\left(e, e^{\prime}\right)$ cross sections of Ref. [92], all in the same kinematics, presented in terms of the ratio defined by Eq.(4.4). . . . . . . . . . . . . . . . . . .

4.3 Comparison between the scaling function of the second kind, $f(\psi)$, obtained from E12-14-012 data on Ar, Ti, and C. The $k_{F}$ of $\mathrm{C}$ is fixed to the value obtained by Moniz et al. [84] while the data analysis of Ti and Ar sets $k_{F}$ at $240 \mathrm{MeV}$ and $245 \mathrm{MeV}$, respectively. The circles are the Ar data from LNF [45], which turn out to prefer an inconsistently higher value of $k_{F}$. . .

4.4 Comparison between the scaling function $F(y)$ obtained from the E12-14-012 data on argon, titanium and carbon, and the argon data obtained at LNF [45]. 72

5.1 Distribution along the beam direction of reconstructed events for the multifoil carbon target. The shaded regions represent the data selected to identify the events coming from individual carbon foils. . . . . . . . . . . . . . 
5.2 Event yields from carbon foils at different positions along the beam direction, normalized to the yield for the central foil, for the uncorrected data and the Monte Carlo simulation. The dependence of the cross section on the scattering angle, correctly taken into account in the Monte Carlo simulation, introduces a linear trend in the data that needs to be corrected for. All uncertainties are purely statistical. . . . . . . . . . . . . . . . . .

5.3 Event yields in the corrected data for the multi-foil carbon target surviving the $z$-position selection, normalized to the yield for the central foil. The outcomes of two correction procedures are compared. The error bars are symmetric and represent the total uncertainties, being the statistical and systematic uncertainties added in quadrature. . . . . . . . . . . . .

5.4 Double-differential cross section extracted for inclusive electron scattering off the Al-7075 target at beam energy $2.222 \mathrm{GeV}$ and scattering angle $15.54^{\circ}$. The inner and outer uncertainty bands correspond to statistical and total uncertainties, respectively. . . . . . . . . . . . . .

5.5 Comparison of the $\mathrm{Al}\left(e, e^{\prime}\right)$ cross sections extracted using the yield-ratio and acceptance methods. The inner (outer) bands for the yield-method show statistical (total) uncertainties. For the acceptance method only statistical uncertainties are shown. . . . . . . . . . . . . . .

5.6 Comparison of the cross sections per nucleon for aluminum, argon [101], titanium [92], and carbon [92] measured at beam energy $2.222 \mathrm{GeV}$ and scattering angle $15.54^{\circ}$. The average nucleon number for every target is calculated according to the natural abundances of isotopes, see details in the text. The bands represent the total uncertainties. . . . . . . . . . . . . . 
5.7 Same as in Fig. 5.6 but for the cross sections normalized by the combination of the elementary cross sections according to Eq. (5.6) . . . . . . . . . 95

5.8 Comparison between the scaling function of aluminum obtained from the E1214-012 data (this work), represented by diamonds, and those obtained from the data of Day et al. [76]. The data are labeled according to the value of $Q^{2}$ corresponding to quasi elastic kinematics. . . . . . . . . . . . . . . . . 98

$5.9 Q^{2}$-dependence of the scaling functions $F\left(y, Q^{2}\right)$ obtained from the cross section displayed in Fig. 5.4 and from the data reported in Ref. [76]. The meaning

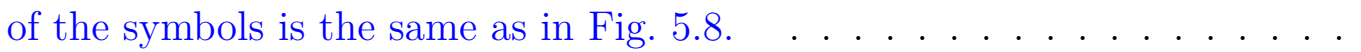

5.10 Scaling functions of second kind, obtained from the inclusive cross sections measured by the E12-14-012 experiment using carbon, aluminum, argon and

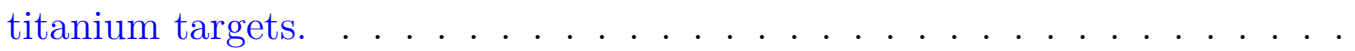

6.1 Missing momentum distributions of protons in argon and titanium assumed

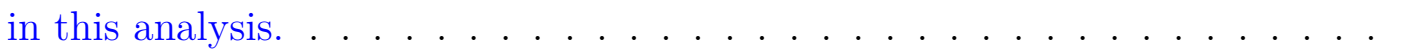

6.2 Missing energy distribution of protons in (a) argon and (b) titanium assumed

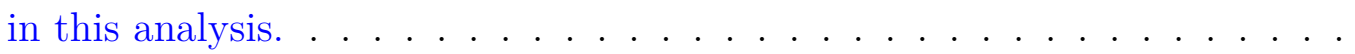

6.3 Six-fold differential cross section as a function of missing energy for argon. The background estimate (line connecting the experimental data points) is multiplied by 10 for purpose of presentation. The MC predictions, based on the mean-field SF, include a correction for the nuclear transparency, while other FSI effects are not accounted for. . . . . . . . . . . . . 
6.4 Six-fold differential cross section as a function of missing energy for titanium. The background estimate (line connecting the experimental data points) is multiplied by 10 for purpose of presentation. The MC predictions, based on the mean-field SF, include a correction for the nuclear transparency, while other FSI effects are not accounted for. . . . . . . . . . . . . . . . 124

6.5 Same as Fig. 6.3 but for the cross section as a function of missing momentum. The inner (outer) uncertainty bands correspond to statistical (total)

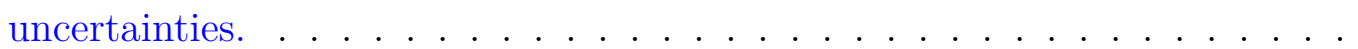

6.6 Same as Fig. 6.3 but for the cross section as a function of missing momentum. The inner (outer) uncertainty bands correspond to statistical (total)

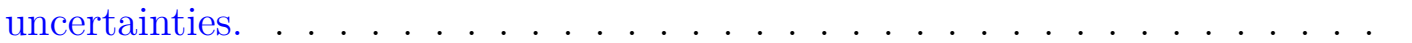

6.7 Reduced cross section as a function of missing momentum for the $1 p_{1 / 2}$ proton knockout from argon. We compare the PWIA and DWIA results obtained for the parallel kinematics considered in this analysis. . . . . . . . . . . .

6.8 Differential cross section for elastic proton scattering on ${ }^{40} \mathrm{Ar}$ at $0.8 \mathrm{GeV}$ as a function of scattering angle. Results for the DEM, EDAD1, and EDAD3 optical potentials, which turn out to almost completely overlap, are compared

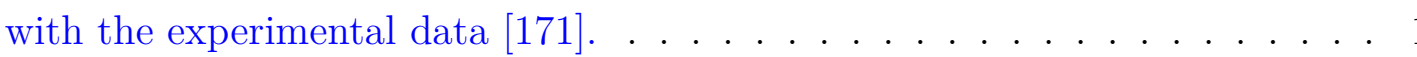

6.9 Six-fold differential cross section for argon as a function of missing momentum integrated over $\quad 0<E_{m}<27 \mathrm{MeV}$. The background estimate is multiplied by 10 for presentation. The MC predictions, based on the mean-field SF, include the full FSI corrections. . . . . . . . . . . . . . . . . . . 132 
6.10 Six-fold differential cross section for argon as a function of missing momentum integrated over $27<E_{m}<44 \mathrm{MeV}$. The background estimate is multiplied by 10 for presentation. The MC predictions, based on the mean-field SF, include the full FSI corrections. . . . . . . . . . . . . . . . . . . . . . 132

6.11 Six-fold differential cross section for argon as a function of missing momentum integrated over $44<E_{m}<70 \mathrm{MeV}$. The background estimate is multiplied by 10 for presentation. The MC predictions, based on the mean-field SF, include the full FSI corrections. . . . . . . . . . . . . . . . . . . . 133

6.12 Same as Fig. 6.9 but for titanium and integrated over $0<E_{m}<30 \mathrm{MeV}$. 133

6.13 Same as Fig. 6.10 but for titanium and integrated over $30<E_{m}<54 \mathrm{MeV} .134$

6.14 Same as Fig. 6.11 but for titanium and integrated over $54<E_{m}<90 \mathrm{MeV} .134$ 


\section{List of Tables}

1.1 Best fit values of the $3 \nu$ oscillation parameters obtained from a global analysis from Ref. [8]. The latter column shows the formal " $1 \sigma$ accuracy" for each parameter, defined as $1 / 6$ of the $3 \sigma$ range divided by the best-fit value (in percent). . . . . . 14

2.1 Hall A HRS - High resolution Spectrometer acceptances and detailed resolutions 35

2.2 Trigger types and trigger logic configurations. . . . . . . . . . . . . 40

2.3 Kinematics settings used to collect the data analyzed here. . . . . . . . . . . 47

3.1 Contributions to the uncertainties associated with the measured $\mathrm{C}\left(e, e^{\prime}\right)$ cross sections. Numbers represents upper limit or range for the uncertainties that vary between kinematical regions. . . . . . . . . . . . . . . . . .

4.1 Uncertainties associated with the presented $\operatorname{Ar}\left(e, e^{\prime}\right)$ cross section. Numbers represent upper limits or the range for the uncertainties that vary between different kinematical regions. . . . . . . . . . . . . . . . . . . .

5.1 Composition of the Al-7075 alloy. For each element, we provide the number of protons $Z$ and the average number of neutrons $N$ calculated according to the isotopic abundances $[102] \ldots \ldots \ldots \ldots \ldots$

5.2 Contributions to systematic uncertainties in the yield-ratio method for aluminum and argon. . . . . . . . . . . . . . . . . 
6.1 Kinematics settings used to collect the data analyzed here. . . . . . . . . . 107

6.2 Trigger lists detailing how the signals from different detector components are combined. LEFT and RIGHT identify the electron and proton arm, respectively.110

6.3 Summary of the efficiency analysis for the argon and titanium targets. . . . 112

6.4 Parametrization of the missing energy distributions of ${ }_{18}^{40} \mathrm{Ar}$ and ${ }_{22}^{48} \mathrm{Ti}$ assumed in this analysis. The central peak position $E_{\alpha}$, its width $\sigma_{\alpha}$, and the lower (upper) bound on the considered energy range, $E_{\text {low }}^{\alpha}\left(E_{\text {high }}^{\alpha}\right)$ are shown for each level $\alpha$. All values are given in units of MeV . . . . . . . . . . . . . 117

6.5 Contributions to systematical uncertainties for argon and titanium. . . . . . 122

6.6 Shifts between the reduced DWIA and PWIA cross sections, and the DWIA to PWIA cross-section ratios, obtained for proton knockout from various argon orbital using different optical potentials: DEM [169], EDAD3 [170], and EDAD1 [170]. All results are calculated for $p_{m}>0 \ldots \ldots \ldots \ldots$ 


\section{Chapter 1}

\section{Introduction and Background}

\section{$1.1 \quad$ Neutrino Oscillation}

Neutrinos are very light, neutral leptons that are one of the fundamental pieces of the standard model of particle physics, and have three different flavors: electron, muon, and tau $\left(\nu_{e}, \nu_{\mu}, \nu_{\tau}\right)$. Though long believed to be massless, observations in the past 20 years have revealed that neutrinos actually oscillate between flavors, indicating that they must have some finite mass (still less than an upper limit of about $2 \mathrm{eV}[6]$ ). This oscillation is a result of the fact that each of the three mass eigenstates is actually a superposition of the three flavor eigenstates, and vice versa.

\subsubsection{Two flavor oscillations}

To explore neutrino oscillation, we first examine the example case of oscillations involving only two mass eigenstates, which we label $|1\rangle$ and $|2\rangle$, and two flavor eigenstates, which we label $|\alpha\rangle$ and $|\beta\rangle$. The relationship between these states can be written:

$$
\left(\begin{array}{c}
\left|\nu_{\alpha}\right\rangle \\
\left|\nu_{\beta}\right\rangle
\end{array}\right)=\left(\begin{array}{cc}
\cos \theta & \sin \theta \\
-\sin \theta & \cos \theta
\end{array}\right)\left(\begin{array}{c}
\left|\nu_{1}\right\rangle \\
\left|\nu_{2}\right\rangle
\end{array}\right)=U\left(\begin{array}{c}
\left|\nu_{1}\right\rangle \\
\left|\nu_{2}\right\rangle
\end{array}\right),
$$


where $U$ is called the mixing matrix and $\theta$ is the mixing angle.

Consider the mass eigenstates $\left|\nu_{k}\right\rangle(k=1,2)$ with mass $m_{k}$, momentum $p$ and energy $E_{k}=$ $\sqrt{p^{2}+m_{k}^{2}}$. In the Schrödinger picture, we have

$$
\left|\nu_{k}(t)\right\rangle=e^{-i E_{k} t}\left|\nu_{k}(0)\right\rangle
$$

The oscillation probability $P\left(\nu_{\alpha} \rightarrow \nu_{\beta}\right)$ can be therefore calculated from the square of the quantum mechanical amplitude

$$
\begin{aligned}
P\left(\nu_{\alpha} \rightarrow \nu_{\beta}\right) & =\left|\left\langle\nu_{\alpha}(0) \mid \nu_{\beta}(t)\right\rangle\right|^{2} \\
& =\left|-\sin \theta \cos \theta\left(e^{-i E_{1} t}-e^{-i E_{2} t}\right)\right|^{2} \\
& =\sin ^{2}(2 \theta) \sin ^{2}\left(\frac{E_{2}-E_{1}}{2} t\right)
\end{aligned}
$$

Since the mass of the neutrino is very small compared to the kinetic energy, for now we will use the relativistic approximation, $E_{k} \approx p+\frac{m_{k}^{2}}{2 p}$. Thus, Eq. (1.3) becomes

$$
P\left(\nu_{\alpha} \rightarrow \nu_{\beta}\right)=\sin ^{2}(2 \theta) \sin ^{2}\left(\frac{\Delta m^{2}}{4 p} t\right),
$$

where $\Delta m^{2}=\left|m_{1}^{2}-m_{2}^{2}\right|$. Since the neutrinos travel approximately at the speed of light, we continue the approximation by using $p \approx E$ and the travel distance $L \approx t$, where we have already let $c=1$ :

$$
P\left(\nu_{\alpha} \rightarrow \nu_{\beta}\right)=\sin ^{2}(2 \theta) \sin ^{2}\left(\Delta m^{2} \frac{L}{4 E}\right) .
$$

Since in the above discussion we have used natural units, to put the formula into practical 
use we shall convert it by substitute $\hbar c$ with $197 \mathrm{eV} \cdot \mathrm{nm}$, so that the equation becomes

$$
P\left(\nu_{\alpha} \rightarrow \nu_{\beta}\right)=\sin ^{2}(2 \theta) \sin ^{2}\left(1.27 \Delta m^{2} \frac{L}{E}\right)
$$

where $L$ is in meters, $E$ is in $M e V$, and $\Delta m^{2}$ is in $e V^{2}$.

\subsubsection{Three flavor oscillations}

In actuality, however, we have three neutrino flavors to consider, as well as three mass eigenstates. The theory of this model was first set down by Pontecorvo [3], and later Maki, Nakagawa, and Sakata [4], for whom the PMNS mixing matrix is named. The following relationship relates the flavor eigenstates $\left|\nu_{\alpha}\right\rangle(\alpha \in\{e, \mu, \tau\})$ to the mass eigenstates $\left|\nu_{i}\right\rangle$ $(i \in\{1,2,3\})$ using the PMNS matrix $\mathrm{U}$ :

$$
\left|\nu_{\alpha}\right\rangle=\sum_{k} U_{\alpha k}\left|\nu_{k}\right\rangle
$$

where the mixing matrix $U$ can be expressed in the form:

$$
\left(\begin{array}{ccc}
c_{12} c_{13} & c_{13} s_{12} & e^{-i \delta} s_{13} \\
-c_{23} s_{12}-e^{i \delta} c_{12} s_{13} s_{23} & c_{12} c_{23}-e^{i \delta} s_{12} s_{13} s_{23} & c_{13} s_{23} \\
s_{12} s_{23}-e^{i \delta} c_{12} c_{23} s_{13} & -e^{i \delta} c_{23} s_{12} s_{13}-c_{12} s_{23} & c_{13} c_{23}
\end{array}\right)
$$

Here $\delta$ is the CP-violating phase, and $c_{i j}$ and $s_{i j}$ are abbreviations of the functions $\cos \theta_{i j}$ and $\sin \theta_{i j}$, where $\theta_{i j}(i, j=1,2,3)$ are mixing angles. 
Using Eq. (1.2) we can write Eq. (1.7) as

$$
\left|\nu_{\alpha}(t)\right\rangle=\sum_{k} U_{\alpha k} e^{-i E_{k} t}\left|\nu_{k}(0)\right\rangle
$$

In order to determine the oscillation probability $P\left(\nu_{\beta} \rightarrow \nu_{\alpha}\right)$, we can first write Eq. (1.7) as

$$
\left|\nu_{k}(0)\right\rangle=\sum_{\beta} U_{\beta k}^{*}\left|\nu_{\beta}(0)\right\rangle
$$

and using Eq. (1.10) and Eq. (1.9), we derive:

$$
\begin{aligned}
\left|\nu_{\alpha}(t)\right\rangle & =\sum_{k, \beta} U_{\alpha k} e^{-i E_{k} t} U_{\beta k}^{*}\left|\nu_{\beta}(0)\right\rangle \\
& =e^{-i p t} \sum_{k, \beta} U_{\alpha k} e^{-i \frac{m_{k}^{2}}{2 p} t} U_{\beta k}^{*}\left|\nu_{\beta}(0)\right\rangle
\end{aligned}
$$

where the relativistic approximation $E_{k} \approx p+\frac{m_{k}^{2}}{2 p}$ is used. Then the oscillation probability can be derived as follows:

$$
\begin{aligned}
P\left(\nu_{\beta} \rightarrow \nu_{\alpha}\right) & =\left|\left\langle\nu_{\beta}(0) \mid \nu_{\alpha}(t)\right\rangle\right|^{2} \\
& =\left|\sum_{i} U_{\alpha k} e^{-i \frac{m_{k}^{2}}{2 p} t} U_{\beta k}^{*}\right|^{2} \\
& =R e \sum_{k, j} U_{\alpha k}^{*} U_{\beta k} U_{\alpha k} U_{\beta k}^{*} e^{-i \frac{m_{k}^{2}-m_{j}^{2}}{2 p} t} \\
& =\delta_{\alpha \beta}-R e \sum_{k, j} U_{\alpha k}^{*} U_{\beta k} U_{\alpha k} U_{\beta k}^{*}\left(1-e^{-i \frac{\Delta m_{k j}^{2}}{2 p} t}\right) \\
& =\delta_{\alpha \beta}-4 \sum_{k>j} U_{\alpha k}^{*} U_{\beta k} U_{\alpha k} U_{\beta k}^{*} \sin ^{2}\left(\frac{\Delta m_{k j}^{2}}{4 p} t\right),
\end{aligned}
$$


where the travel distance $L$ is approximately equal to the time period $t$ in natural units:

$$
P\left(\nu_{\beta} \rightarrow \nu_{\alpha}\right)=\delta_{\alpha \beta}-4 \sum_{k>j} U_{\alpha k}^{*} U_{\beta k} U_{\alpha k} U_{\beta k}^{*} \sin ^{2}\left(\frac{\Delta m_{k j}^{2} L}{4 p}\right)
$$

\subsection{Neutrino oscillation experiments}

Using the above equation (Eq. (1.13)) then, neutrino oscillation experiments frequently seek to determine the oscillation parameters by observing the percentage of neutrinos which have oscillated to different flavors over some fixed baseline L. This can take the form of either appearance or disappearance searches, where one starts with a pure beam of one neutrino flavor, and measures either the appearance of the other two flavors, or the disappearance of the original flavor, between a near and a far detector. In an arbitrary oscillation of $\nu_{\alpha} \rightarrow \nu_{\beta}$, we can write down the event rates at the near and far detectors as follows [90]:

$$
\begin{gathered}
N_{\mathrm{ND}}^{\alpha}\left(\boldsymbol{p}_{\text {reco }}\right)=\sum_{i} \phi_{\alpha}\left(E_{\text {true }}\right) \times \sigma_{\alpha}^{i}\left(\boldsymbol{p}_{\text {true }}\right) \times \epsilon_{\alpha}\left(\boldsymbol{p}_{\text {true }}\right) \times R_{i}\left(\boldsymbol{p}_{\text {true }} ; \boldsymbol{p}_{\text {reco }}\right) \\
N_{\mathrm{FD}}^{\alpha \rightarrow \beta}\left(\boldsymbol{p}_{\text {reco }}\right)=\sum_{i} \phi_{\alpha}\left(E_{\text {true }}\right) \times P_{\alpha \beta}\left(E_{\text {true }}\right) \times \sigma_{\beta}^{i}\left(\boldsymbol{p}_{\text {true }}\right) \times \epsilon_{\beta}\left(\boldsymbol{p}_{\text {true }}\right) \times R_{i}\left(\boldsymbol{p}_{\text {true }} ; \boldsymbol{p}_{\text {reco }}\right) .
\end{gathered}
$$

Here $N_{\mathrm{ND}}^{\alpha}\left(\boldsymbol{p}_{\text {reco }}\right)$ and $N_{\mathrm{FD}}^{\alpha}\left(\boldsymbol{p}_{\text {reco }}\right)$ represent the event rate at the near/far detectors as a function of the reconstructed variable $\boldsymbol{p}_{\text {reco }} \equiv\left(E_{\text {reco }}, \vec{p}_{\text {reco }}\right), \phi_{\alpha}$ is the flux of $\alpha$ neutrinos at the near detector, $P_{\alpha \beta}\left(E_{\text {true }}\right)$ is the oscillation probability, $\sigma_{\alpha}^{i}$ is the neutrino cross section for flavor $\alpha$ at interaction $i, \epsilon_{\alpha}$ is the detector efficiency for a neutrino of flavor $\alpha$ interacting in the detector, and $R_{i}\left(\boldsymbol{p}_{\text {true }} ; \boldsymbol{p}_{\text {reco }}\right)$ is the probability that an event with $\boldsymbol{p}_{\text {true }}$ is reconstructed with a different value of its momentum $\left(\boldsymbol{p}_{\text {reco }}\right)$ due to nuclear or detector effects.

The oscillation probability $P_{\alpha \beta}$ is determined through taking the ratio between these two 
equations (Eq. (1.15) and Eq. (1.14)). However, the neutrino energy $E_{\text {true }}$ cannot be measured directly, owing to the light, neutral nature of the neutrino, and we must reconstruct it from the variables we can observe, as discussed in Sec. (1.2.2). The factors in (1.15) and (1.14) which depend strongly on the neutrino energy are the neutrino flux distribution, the cross sections, and the detector effects, so it is important to be sure the reconstruction is accurate.

Future neutrino oscillation experiments including DUNE (Deep Underground Neutrino Experiment) will use time projections chambers (TPCs) with liquid argon as a scattering target for incoming neutrinos. As there is currently no reliable cross section model for argon, we seek to develop one via an electron scattering experiment (E12-14-012) at Jefferson Lab. The insights that this experiment will provide into the nuclear model of argon can then be applied to neutrino scattering off an argon target. Broadly, electron scattering can provide the vector part of the neutrino scattering current, and the additional axial part can be computed theoretically or measured directly. The details of this are described more fully in the next section.

\subsubsection{Neutrino-nucleus cross section}

In charged-current neutrino-nucleus interactions, the interaction process can be written as

$$
\nu_{\ell}(k)+A(p) \rightarrow \ell^{-}\left(k^{\prime}\right)+X\left(p^{\prime}\right)
$$

where a neutrino $\nu_{\ell}$ scatters off a nuclear target A, and in the final state, there is an outgoing lepton $\ell$ and a hadronic state $\mathrm{X}$. The four-momenta can be defined as

$$
k=\left(E_{\nu}, \mathbf{k}\right), k^{\prime}=\left(E_{\ell}, \mathbf{k}^{\prime}\right), p=\left(E_{p}, \mathbf{p}\right), p^{\prime}=\left(E_{p^{\prime}}, \mathbf{p}^{\prime}\right)
$$


The double differential cross section of the process can then be written in the form $[40,50$, $51,54]$

$$
\frac{d^{2} \sigma}{d \Omega_{\ell} d E_{\ell}}=\frac{G_{F}^{2} V_{u d}^{2}}{16 \pi^{2}} \frac{\left|\mathbf{k}_{\ell}\right|}{\left|\mathbf{k}_{\nu}\right|} L_{\lambda \mu} W^{\lambda \mu}
$$

where $G_{F}$ is the Fermi constant, and $\left|V_{u d}\right|$ is the coupling of the $\mathrm{u}$ and $\mathrm{d}$ quarks in the Cabibbo-Kobayashi-Maskawa (CKM) matrix $V . L_{\lambda \mu}$ and $W^{\lambda \mu}$ are respectively the leptonic tensor and hadronic tensor. The leptonic tensor can be defined as

$$
L_{\lambda \mu}=8\left(k_{\ell}^{\lambda} k_{\nu}^{\mu}+k_{\mu}^{\lambda} k_{\ell}^{\nu}-g^{\lambda \mu} k_{\ell} \cdot k_{\nu}-i \epsilon^{\lambda \mu \alpha \beta} k_{\ell \beta} k_{\nu \alpha}\right)
$$

where all masses of leptons are neglected. $g_{\lambda \nu}$ is the metric tensor and $\epsilon_{\lambda \nu \alpha \beta}$ is the fully antisymmetric Levi-Civita tensor. The hadronic tensor is defined by

$$
W^{\lambda \nu}=\sum_{X} \frac{1}{2 E_{p}}\left\langle 0(p)\left|J^{\lambda}\right| X\left(p^{\prime}\right)\right\rangle\left\langle X\left(p^{\prime}\right)\left|J^{\nu}\right| 0(p)\right\rangle \delta\left((p+q)^{2}-m_{N}^{\prime 2}\right),
$$

where $m_{N}^{\prime}$ is the mass of the final state and $q$ is the four-momentum transferred, $q=p^{\prime}-p$. In the most general case, $W_{\lambda \nu}$ can be written as a function of five different structure functions [40]:

$$
\begin{aligned}
W^{\lambda \mu}=-g^{\lambda \mu} W_{1}+\frac{p^{\lambda} p^{\mu}}{m_{N}^{2}} W_{2} & -i \epsilon^{\lambda \mu \varrho \sigma} \frac{p_{\varrho} q_{\sigma}}{m_{N}^{2}} W_{3} \\
& +\frac{q^{\lambda} q^{\mu}}{m_{N}^{2}} W_{4}+\frac{p^{\lambda} q^{\mu}+q^{\lambda} p^{\mu}}{m_{N}^{2}} W_{5} .
\end{aligned}
$$

From Eq. (1.19) and Eq. (1.21), we obtain [54] 


$$
L^{\lambda \mu} W_{\lambda \mu}=16 \sum_{i} W_{i}\left(\frac{A_{i}}{m_{N}^{2}}\right)
$$

where the $A_{i}$ 's are:

$$
\begin{aligned}
& A_{1}=m_{N}^{2}\left(k \cdot k^{\prime}\right) \\
& A_{2}=(k \cdot p)\left(k^{\prime} \cdot p\right)-\frac{A_{1}}{2} \\
& A_{3}=(k \cdot p)\left(k^{\prime} \cdot q\right)-(k \cdot q)\left(k^{\prime} \cdot p\right), \\
& A_{4}=(k \cdot q)\left(k^{\prime} \cdot q\right)-\frac{q^{2}}{2} \frac{A_{1}}{m_{N}^{2}}, \\
& A_{5}=(k \cdot p)\left(k^{\prime} \cdot q\right)+\left(k^{\prime} \cdot p\right)(k \cdot q)-(q \cdot p) \frac{A_{1}}{m_{N}^{2}},
\end{aligned}
$$

in the quasi-elastic channel, the structure functions $W_{i}$ 's are given by :

$$
\begin{aligned}
& W_{1}=2\left[F_{A}^{2}(1+\tau)+\tau\left(F_{1}+F_{2}\right)^{2}\right], \\
& W_{2}=2\left[F_{A}^{2}+F_{1}^{2}+\tau F_{2}^{2}\right], \\
& W_{3}=2\left[F_{A}\left(F_{1}+F_{2}\right)\right], \\
& W_{4}=\left[F_{2}^{2}(1+\tau)-2 F_{2}\left(F_{1}+F_{2}\right)\right] / 2, \\
& W_{5}=W_{2} / 2,
\end{aligned}
$$

with $\tau=\frac{-q^{2}}{4 m_{N}^{2}}$, where $F_{1}$ and $F_{2}$ are vector form factors, and $F_{A}$ is the axial-vector form factor. Ref. [54] contains a more detailed and complete derivation of the above equations.

The expressions for the structure functions for the resonance production and deep inelastic scattering channels can be found in Ref. [40]. 


\subsubsection{Neutrino Energy Reconstruction}

The determination of the neutrino oscillation parameters depends from the neutrino energy reconstruction. Neutrino beam are not monochromatic, and the neutrino energy has to reconstructed event by event. Neutrino are produced by primary proton beams that hit a target reach in protons. Pions and Kaons are produced during those interactions and their subsequent 3-body decay produce neutrinos. A pulsing magnet (horn) focus positively(negatively) charged pions and defocus negatively(positively) charged pions in case of the neutrino(antineutrino) beam. As a result the energy spectrum is quite broad. The event by event neutrino energy is determined by measuring the kinematics of the outgoing particles.

Considering, for example, the simplest case of charged current quasielastic scattering (CCQE) we have

$$
\nu_{\ell}+A \rightarrow \ell^{-}+X
$$

here a neutrino with four momentum $k=\left(E_{\nu}, \mathbf{k}\right)$ scatters off a nuclear target $\mathrm{A}$, and in the final state, an outgoing lepton with four momentum $k^{\prime}=\left(E_{\ell}, \mathbf{k}^{\prime}\right)$ and an hadronic state $\mathrm{X}$ are produced.

Applying energy and momentum conservations, the kinematic energy reconstruction for charged current quasielastic (CCQE) can be determined:

$$
E_{\nu}=\frac{m_{p}^{2}-m_{\ell}^{2}-E_{n}^{2}+2 E_{\ell} E_{n}-2 \mathbf{k}_{n} \cdot \mathbf{p}_{n}+\left|\mathbf{p}_{n}^{2}\right|}{2\left(E_{n}-E_{\ell}+\left|\mathbf{k}_{\ell}\right| \cos \theta_{\ell}-\left|\mathbf{p}_{n}\right| \cos \theta_{n}\right)}
$$

where $\left|\mathbf{k}_{\ell}\right|$ and $\left|\theta_{\ell}\right|$ is measured in the detector and $\mathbf{p}_{n}$ and $E_{n}$ are momentum and energy of 
the interacting neutrons which has to be determined using a nuclear model.

The spectral function(SF)is a particular nuclear model that describes neutrino-nucleus interactions. In the SF model, $P\left(P_{m}, E_{m}\right)$ is the probability of finding a nucleon with initial momentum $P_{m}$ and energy $E_{m}$ in the nucleus.

In existing simulation codes the following approximation is used:

$$
\left|p_{n}\right|=0, E_{n}=m_{n}-\epsilon,
$$

that is also known as Fermi Gas (FG) model. In the FG model, each nucleon is considered isolated and not interacting with other nucleons. In FG, the initial momentum of all nucleons is uniformly distributed and it is less than the Fermi momentum $K_{F}$. The removal energy is considered constant. Both $K_{F}$ and the removal energy depend on the target nucleus.

In $\mathrm{SF}$ model, the nucleon-nucleon $(\mathrm{N}-\mathrm{N})$ interactions are included, and so the momentum distribution is highly correlated with the energy of the nucleon and not constant like in the FG model.

Figure 1.1 shows the neutrino energy reconstructed from Eq. (1.26) using $2 \times 10^{4}\left(\left|\mathbf{p}_{n}\right|, E_{n}\right)$ values sampled from the probability distributions associated with the SF model and FG model for the oxygen nuclei.

Figure 1.1 shows differences in the reconstructed neutrino energy for a beam of $E_{\mu}=600 \mathrm{MeV}$ and angle $\theta_{\mu}=60^{\circ}$. This is similar to the MiniBooNE and T2K experiment neutrino beam. The average reconstructed neutrino energy using the SF model is shifted to higher values with respect to the prediction from the FG model by about $20 \mathrm{MeV}$. The bottom of Fig. 1.1 shows the same effect but for a beam with $E_{\mu}=1 \mathrm{GeV}$. In this latter case the shift is slightly bigger, $\sim 70 \mathrm{MeV}$ [5]. 


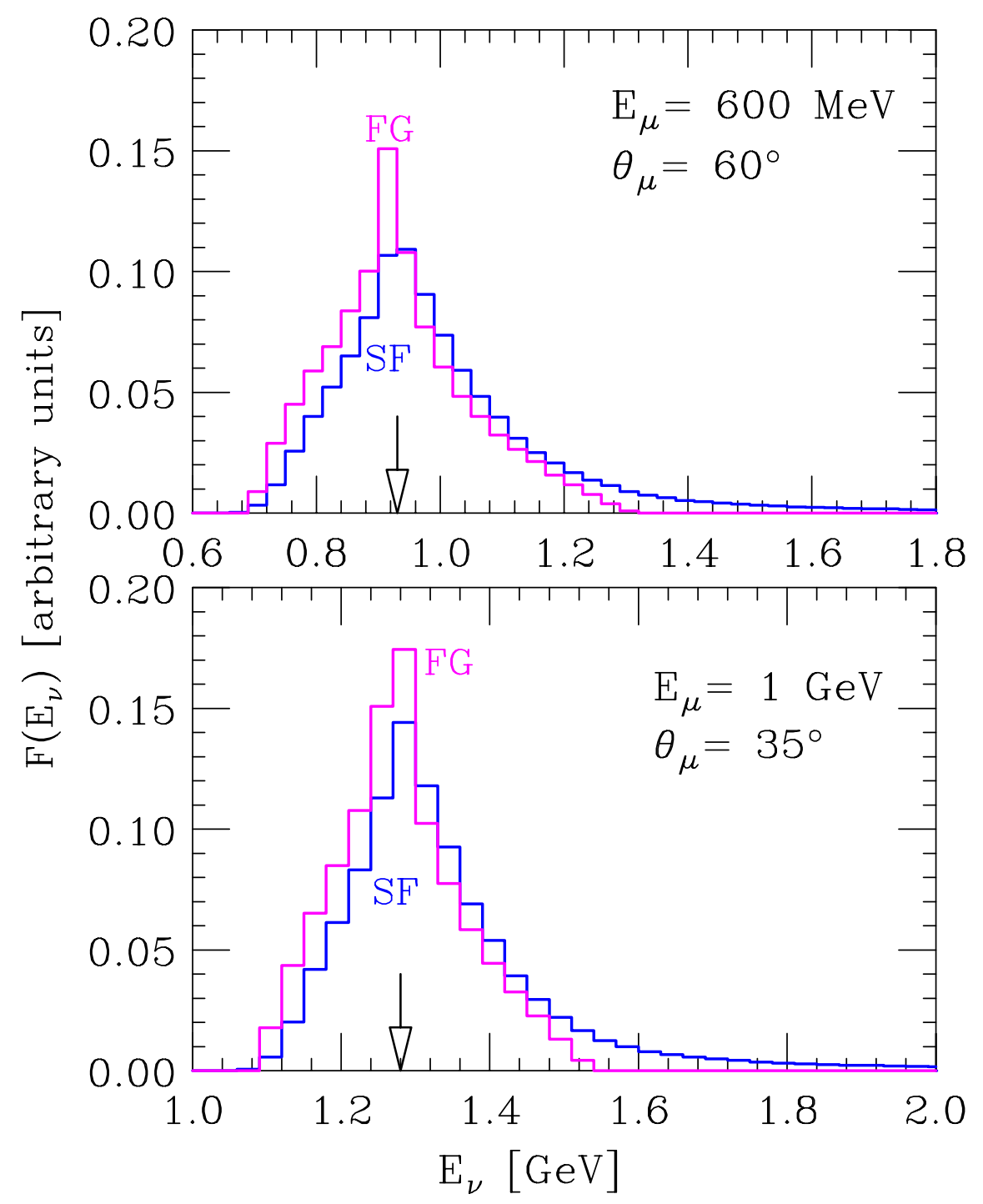

Figure 1.1: Reconstructed neutrino energy distributions using the FG model and SF model. The plot on the top shows the results at $E_{\mu}=600 \mathrm{MeV}$ and $\theta_{\mu}=60^{\circ}$, on the bottom is at $E_{\mu}=1 \mathrm{GeV}$ and $\theta_{\mu}=35^{\circ}[5]$.

Figure 1.2 shows the effect of nuclear models on a DUNE-like experiment. If the reconstructed neutrino energy is underestimated for example by $\sim 20 \%$, in a DUNE-like experiment there would be a significant bias in the extracted $\delta_{C P}$ phase. In the case of $30 \%$ energy underestimation, the extracted $\delta_{C P}$ would exclude the true value at a level between 2 and $3 \sigma$ [40]. 


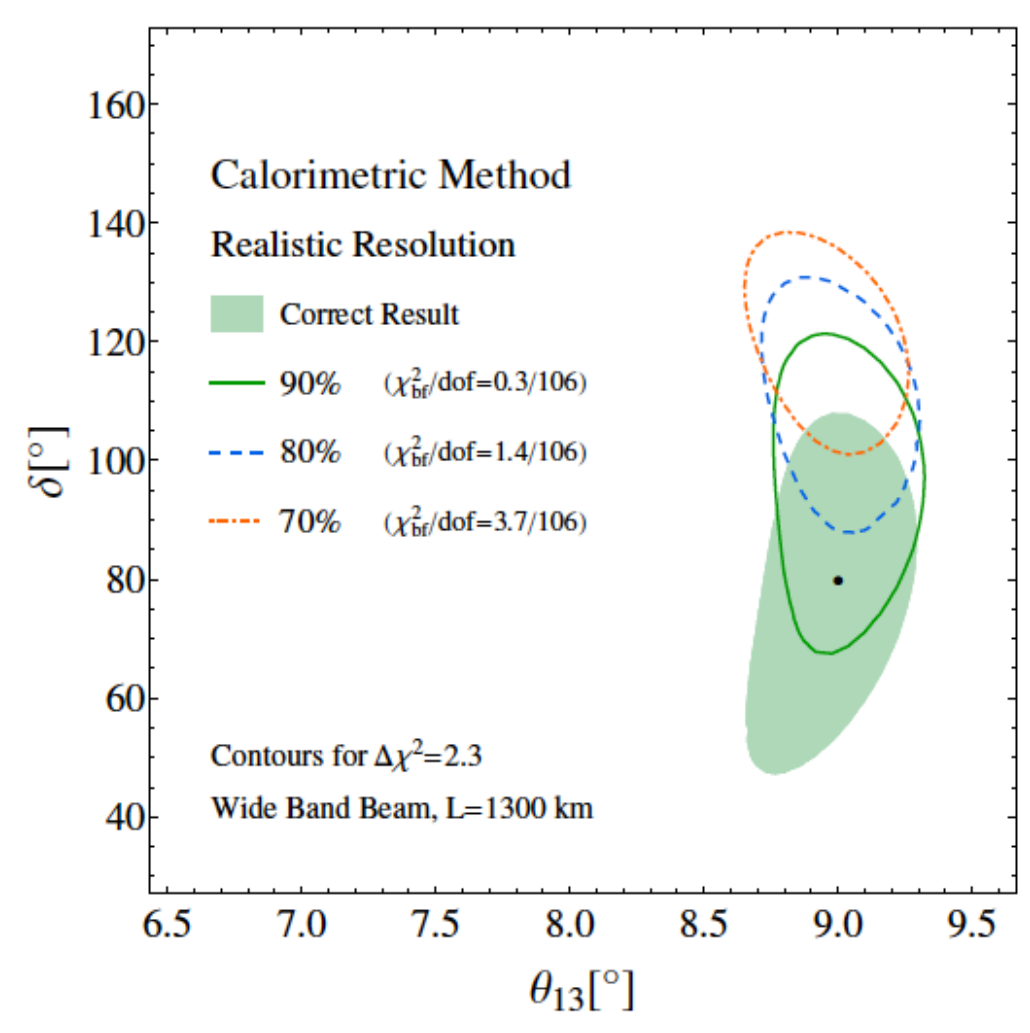

Figure 1.2: $1 \sigma$ confidence regions of $\left(\theta_{13}, \delta_{C P}\right)$ using data from simulation. The results are shown when the migration matrices accounting for $90 \%, 80 \%$, and $70 \%$ of the missing energy, while the green area shows the case for all of the missing energy. The true values of $\theta_{13}$ and $\delta_{C P}$ are shown as the black dot in the center of the green area [40].

\subsubsection{Past Experimental Oscillation Results}

Table 1.1 shows a summary of the most recent neutrino oscillation parameter measurements. Table 1.1 includes various data sets, including solar and long-and short-baseline reactor experiments, short- and long-baseline accelerator experiments, and atmospheric neutrino results [8].

$\delta m^{2}$ and $\Delta m^{2}$ are defined as

$$
\delta m^{2}=m_{2}^{2}-m_{1}^{2}>0, \Delta m^{2}=m_{3}^{2}-\frac{m_{1}^{2}+m_{2}^{2}}{2}
$$




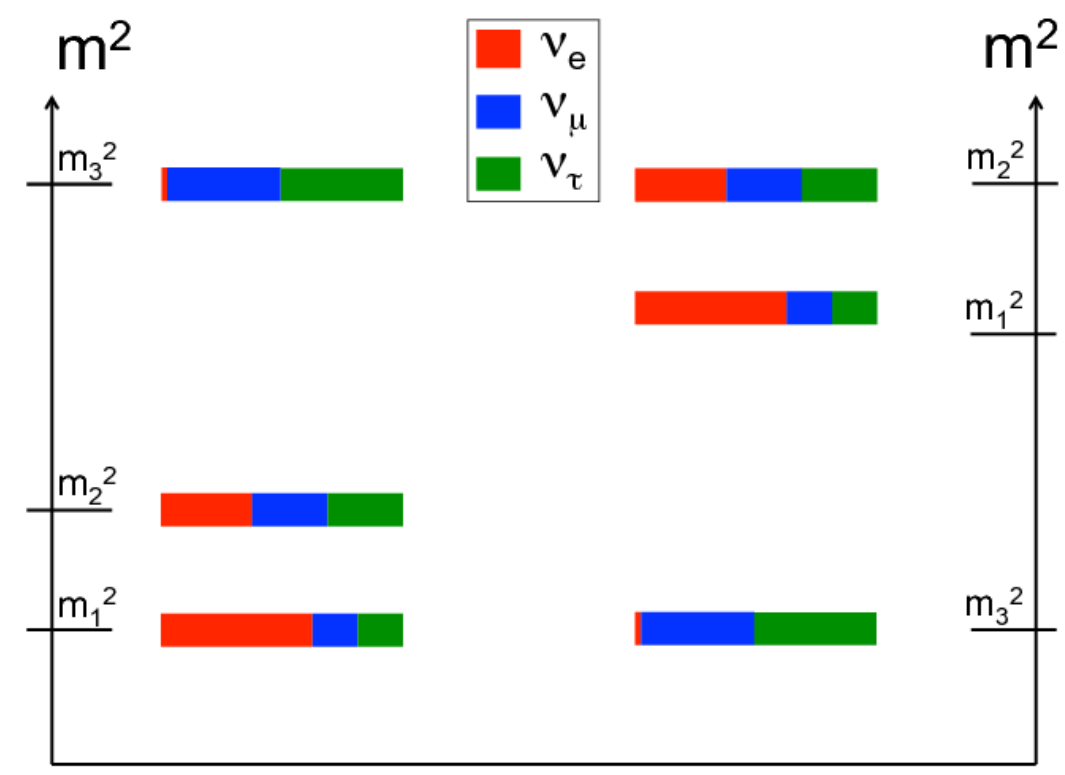

Normal Ordering (NO) Inverted Ordering (IO)

Figure 1.3: Schematic of the normal and inverted hierarchy definition [7]. In the plot the different colors correspond to the flavor contents for the corresponding neutrino mass eigenstates.

To be noted that oscillation neutrino experiments could only determine the squared difference of the neutrino masses, so there is an ambiguity between possible mass hierarchies, if $\Delta m^{2}>$ 0 , then we have the normal ordering (NO), otherwise we will have inverted ordering (IO) as in Fig. 1.3.

\subsubsection{Deep Underground Neutrino Experiment - DUNE}

The US DUNE [11] experiment is the next big long baseline neutrino experiment to be build in US. DUNE is an international collaboration with more than 1,200 physicists involved and more than 35 countries. DUNE will aim to discover charge parity (CP) violation in the neutrino sector and at the same time will have a rich physics program that will involve nucleon decay, neutron-anti-neutron oscillation and supernovae physics. 
Table 1.1: Best fit values of the $3 \nu$ oscillation parameters obtained from a global analysis from Ref. [8]. The latter column shows the formal " $1 \sigma$ accuracy" for each parameter, defined as $1 / 6$ of the $3 \sigma$ range divided by the best-fit value (in percent).

\begin{tabular}{lcccccc}
\hline \hline Parameter & Ordering & Best fit & $1 \sigma$ range & $2 \sigma$ range & $3 \sigma$ range & " $1 \sigma "(\%)$ \\
\hline$\delta m^{2} / 10^{-5} \mathrm{eV}^{2}$ & NO & 7.34 & $7.20-7.51$ & $7.05-7.69$ & $6.92-7.91$ & 2.2 \\
& IO & 7.34 & $7.20-7.51$ & $7.05-7.69$ & $6.92-7.91$ & 2.2 \\
\hline $\sin ^{2} \theta_{12}$ & NO & 3.04 & $2.91-3.18$ & $2.78-3.32$ & $2.65-3.46$ & 4.4 \\
& IO & 3.03 & $2.90-3.17$ & $2.77-3.31$ & $2.64-3.45$ & 4.4 \\
\hline $\sin ^{2} \theta_{13} / 10^{-2}$ & NO & 2.14 & $2.07-2.23$ & $1.98-2.31$ & $1.90-2.39$ & 3.8 \\
& IO & 2.18 & $2.11-2.26$ & $2.02-2.35$ & $1.95-2.43$ & 3.7 \\
\hline$\left|\Delta m^{2}\right| / 10^{-3} \mathrm{eV}^{2}$ & NO & 2.455 & $2.423-2.490$ & $2.390-2.523$ & $2.355-2.557$ & 1.4 \\
& IO & 2.441 & $2.406-2.474$ & $2.372-2.507$ & $2.338-2.540$ & 1.4 \\
\hline $\sin ^{2} \theta_{23} / 10^{-1}$ & NO & 5.51 & $4.81-5.70$ & $4.48-5.88$ & $4.30-6.02$ & 5.2 \\
& IO & 5.57 & $5.33-5.74$ & $4.86-5.89$ & $4.44-6.03$ & 4.8 \\
\hline$\delta / \pi$ & NO & 1.32 & $1.14-1.55$ & $0.98-1.79$ & $0.83-1.99$ & 14.6 \\
& IO & 1.52 & $1.37-1.66$ & $1.22-1.79$ & $1.07-1.92$ & 9.3 \\
\hline \hline
\end{tabular}

The DUNE experiment will have a neutrino beam and a near detector complex at the Fermi National Accelerator Laboratory (Fermilab) and its far detector will be more than 800 miles away in South Dakota. The DUNE far detector will be placed at 4,850 feet underground in the Sanford underground research facility (SURF). DUNE is expected to start the construction phase for the far detector in 2026 and will collect neutrino and antineutrino interactions for more than 10 years. The data taking is expected for 2028 .

The DUNE near detector will measure the neutrino flux, energy, and cross sections on multiple targets including Ar. The DUNE far detector will deploy a Liquid Argon Time Projection Chamber (LArTPC), which will provide high precision identification of neutrino flavors, allowing precision measurements of the $\mathrm{CP}$ violation phase, and advance protondecay lifetime limits $[10,11]$.

The Long-Baseline Neutrino Facility (LBNF) hosted at Fermilab, will provide the neutrino beam. LBNF will generate a wide-band, high purity neutrino and antineutrino beams using 


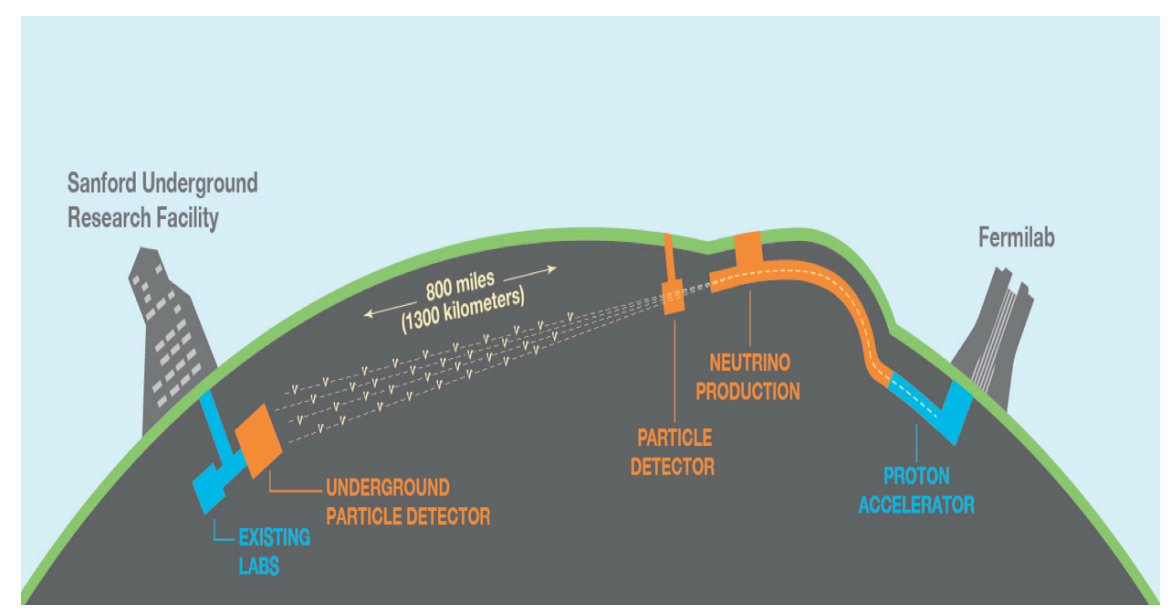

Figure 1.4: Schematic of the Deep Underground Neutrino Experiment [10].

proton energy ranging from 60 to $120 \mathrm{GeV}$. The corresponding neutrino energy will be between $1 \mathrm{GeV}$ and $10 \mathrm{GeV}$.

DUNE will be able to perform many high precision measurements: by measuring the $\nu_{\mu} \rightarrow \nu_{e}$ and $\overline{\nu_{\mu}} \rightarrow \overline{\nu_{e}}$ oscillation probabilities, DUNE will provide precision measurement of the third mixing angle $\theta_{13}$, measurement of the charge-parity $(\mathrm{CP})$ violation phase $\delta_{C P}$, and determination of the sign of $\Delta m_{31}^{2}$ and the mass hierarchy [10, 11].

The CP violation phase $\delta_{C P}$ will be measured through"appearance" channel. DUNE will observe oscillations of $\nu_{\mu} \rightarrow \nu_{e}$ and $\bar{\nu}_{\mu} \rightarrow \bar{\nu}_{e}$. It will not measure the $\nu_{\mu} \rightarrow \nu_{\tau}$ or $\bar{\nu}_{\mu} \rightarrow \bar{\nu}_{\tau}$ because the $\nu_{\tau}$ detection is difficult.

$\mathrm{CP}$ violations implies that there is a difference between the oscillation probabilities for neutrino $P\left(\nu_{\mu} \rightarrow \nu_{e}\right)$ and anti-neutrino $P\left(\bar{\nu}_{\mu} \rightarrow \bar{\nu}_{e}\right)$. The neutrino oscillation probability depends on the neutrino energy $\left(E_{\nu}\right)$, the distance $L$ between near and far detector (baseline) and the oscillation parameters $\Delta m^{2}$ and $\sin ^{2} \theta_{23}$.

In the case of DUNE the baseline is of $L=800$ miles, the dependences of the oscillation probability on the neutrino energy $E_{\nu}$ is shown in Fig. $(1.5,1.6)$. In Figures $(1.5,1.6)$ the 

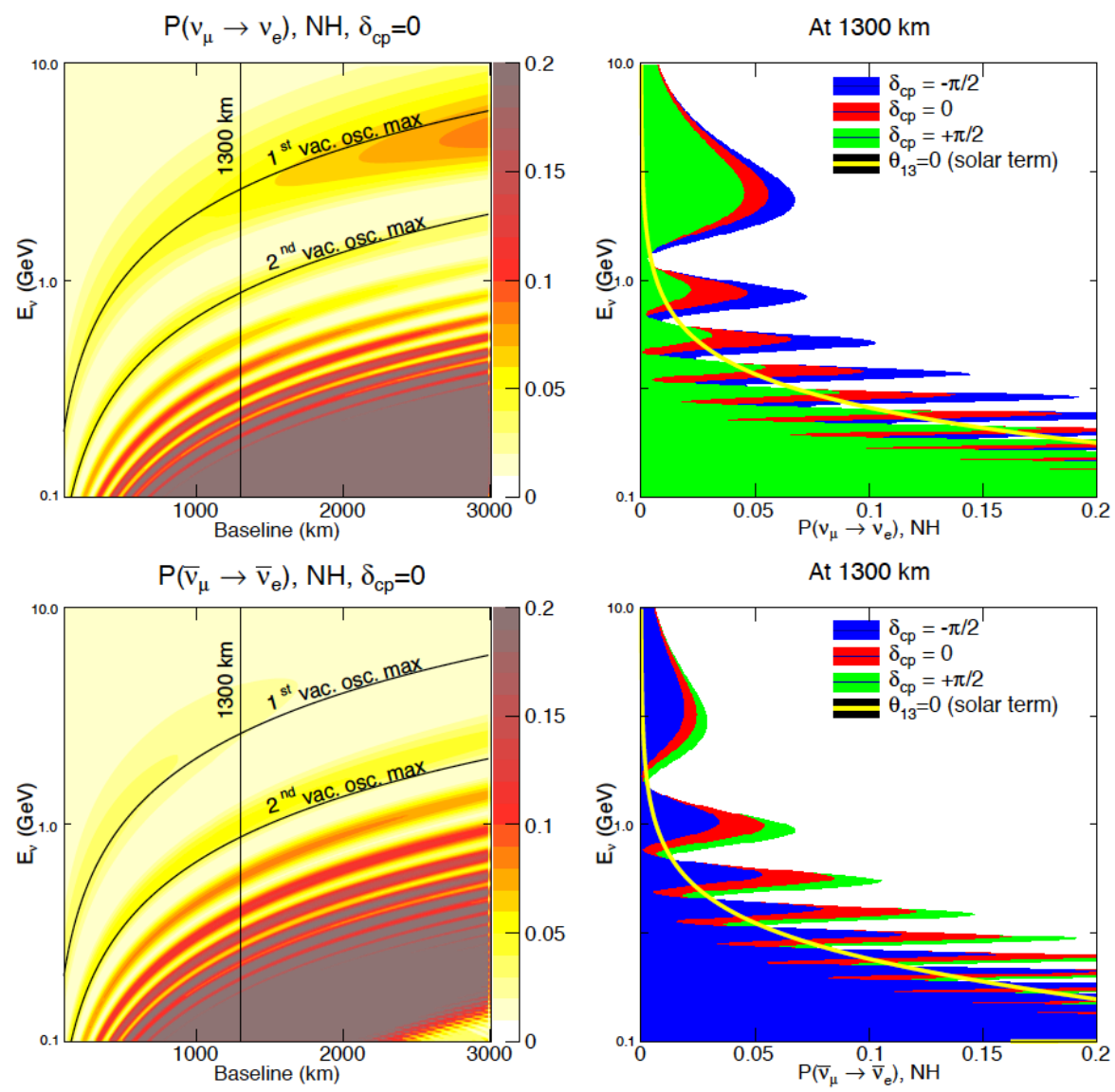

Figure 1.5: Top-left plot represents the neutrino oscillation probability $P\left(\nu_{\mu} \rightarrow \nu_{e}\right)$ as a function of $L$ and $E_{\nu}$ with a value for $\delta_{C P}=0$ and assume NO for the mass hierarchy. The top right plot shows $P\left(\nu_{\nu} \rightarrow \nu_{e}\right)$ as a function of $E_{\nu}$ at the DUNE baseline (800 miles) for $\delta_{C P}=0,-\pi / 2$, and $\pi / 2$. The bottom plots show the same but for antineutrinos $\bar{\nu}_{\mu} \rightarrow \bar{\nu}_{e}[10]$.

different colors are used to indicate different values of $\delta_{C P}[10]$.

By measuring the shape of the oscillation probabilities as a function of the neutrino energies the DUNE energy it is possible to determine the value of $\delta_{C P}$. 

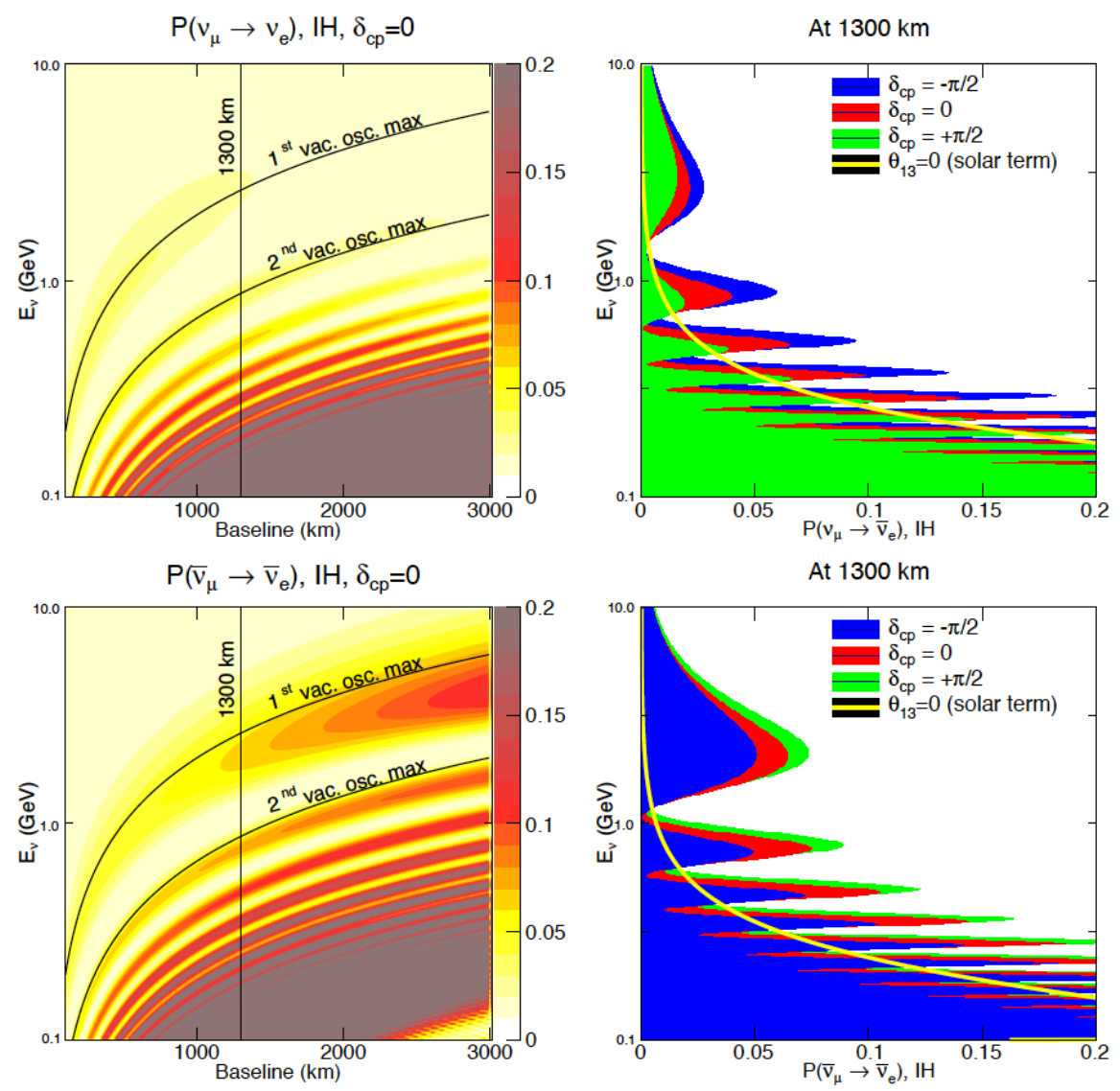

Figure 1.6: Same as Fig. 1.5 but assuming IO for the mass hierarchy [10].

\subsection{Electron scattering}

In the past, to study a nuclear structure of the nucleus, electron scattering has been of paramount importance. Electrons are a clean probe for understanding nuclear structure and they can be produced in abundance. The electron scattering process is an exchange of virtual photons between the probe (the electron) and the target nucleus. A virtual photon with a fixed energy $(\omega)$ and 3-momentum $(q)$ could interact with charge density and electromagnetic currents of the target nucleus. Details of the nuclear structure can be determined by measuring the cross section of electron scattering at various angle and momentum. 
The main goal of the E12-14-012 experiment is to measure the exclusive $\operatorname{Ar}\left(\mathrm{e}, \mathrm{e}^{\prime} \mathrm{p}\right)$ and $\operatorname{Ti}\left(\mathrm{e}, \mathrm{e}^{\prime} \mathrm{p}\right)$ cross sections and determine the proton and neutron spectral functions of natural argon and natural titanium nuclei. There is no previous data available for neither argon and titanium. The experiment took data in 2017 to measure both the inclusive (e,e') and the exclusive $\left(e, e^{\prime} p\right)$ cross sections. The inclusive cross section provides important information such as the integral of the spectral function, which is the sum of contributions from all possible channels. It gives less specific information than the exclusive scattering, but provides information over a broader range of momentum and energy.

\subsubsection{Inclusive cross section $\left(\mathrm{e}, \mathrm{e}^{\prime}\right)$}

There are three main ingredients in an inclusive electron scattering experiment: the beam, the target and a spectrometer that detects the scattered electron. The angle of the spectrometer and its optics determine the outgoing electron momentum. The inclusive channel includes all of the processes that can produce an electron in final state and does not select any specific reaction so the classification of "inclusive" measurement. The $\left(\mathrm{e}, \mathrm{e}^{\prime}\right)$ cross section as a function of the energy transfer $\omega$ for a fixed value of $Q^{2}=q^{2}-\omega^{2}$ [12] is shown in Fig. 1.7. In Fig. 1.7 the first peak on the left is the elastic peak, at this peak, the energy transfer $\omega=\frac{Q^{2}}{2 m_{A}}$ (where $m_{A}$ is the nuclear mass) is very low, and the virtual photon interacts with the entire nucleus. As the $\omega$ becomes higher, the target nucleus could be excited to higher energy bound states, and that corresponds to the few sharp peaks that followed the elastic peak on the right. If the $\omega$ is increased even more, then we reach the collective mode of excitation of the nucleus, also called the "Giant Resonances". The quasielastic peak is a

broad peak and it is centered near the $\omega$ value of $\frac{Q^{2}}{2 m}$ (where $m$ is the mass of the nucleon), which corresponds to the fact that the virtual photon has been absorbed by a single nucleon. Increasing the energy transfer even more, we can see in Fig. 1.7 the $\Delta$ and $N^{*}$ resonances. In 


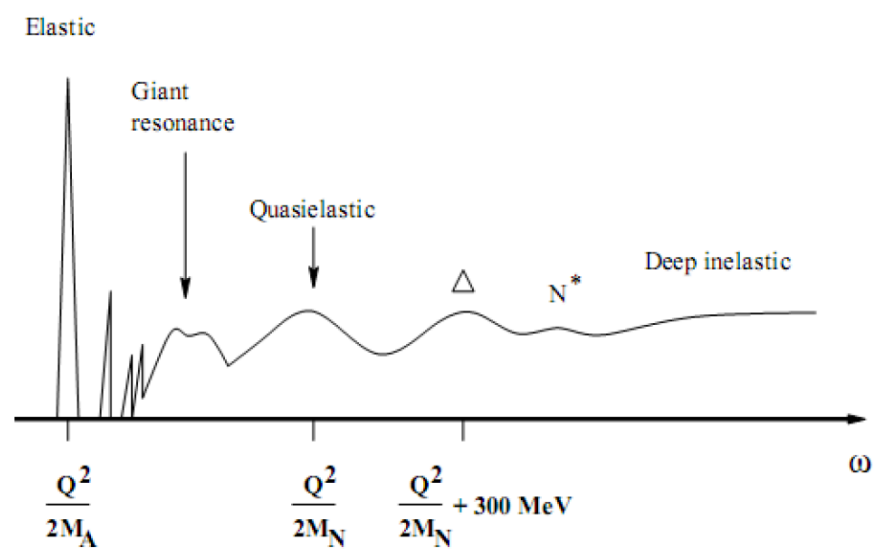

Figure 1.7: General (e,e') energy spectrum

this region, the quarks inside the nucleon absorb the virtual photons, and the nucleon gets excited. At very high value for the $\omega$, we get to the region called "Deep Inelastic Scattering", where the electron breakup of the nucleon. For an inclusive electron scattering process

$$
e\left(k_{e}\right)+A(p) \rightarrow e^{\prime}\left(k_{e^{\prime}}\right)+X\left(p^{\prime}\right)
$$

where the electron scatters off a nuclear target $A$, and only the scattered electron is detected. The four-momenta are defined as

$$
k_{e}=\left(E_{e}, \mathbf{k}_{e}\right), k_{e^{\prime}}=\left(E_{e^{\prime}}, \mathbf{k}_{e^{\prime}}\right), p=\left(E_{p}, \mathbf{p}\right), p^{\prime}=\left(E_{p^{\prime}}, \mathbf{p}^{\prime}\right) .
$$

In the approximation of just one photon exchange, the differential cross section is

$$
\frac{d^{2} \sigma}{d \Omega_{e^{\prime}} d E_{e^{\prime}}}=\frac{\alpha^{2}}{Q^{4}} \frac{E_{e^{\prime}}}{E_{e}} L_{\lambda \mu} W^{\lambda \mu}
$$

where $d \Omega_{e^{\prime}}$ is the differential solid angle into where the electron scatters, $Q^{2}=-q^{2}$ and $q=k_{e}-k_{e^{\prime}} \equiv(\omega, \mathbf{q})$ is the four momentum transfer. 
The tensor $L_{\lambda \mu}$ could be cast in the form

$$
L_{\lambda \mu}=2\left[k_{e}^{\lambda} k_{e}^{\mu}+k_{e^{\prime}}^{\mu} k_{e^{\prime}}^{\lambda}-g^{\lambda \mu}\left(k_{e} k_{e^{\prime}}\right)\right]
$$

where the electron mass is neglected and $g^{\lambda \mu}=\operatorname{diag}(1,-1,-1,-1)$.

The tensor $W^{\lambda \mu}$ is defined by

$$
W^{\lambda \mu}=\sum_{X}\left\langle 0\left|J^{\lambda}\right| X\right\rangle\left\langle X\left|J^{\mu}\right| 0\right\rangle \delta^{(4)}\left(p+q-p^{\prime}\right)
$$

In general, $W^{\lambda \mu}$ can be written as a function of two distinct structure functions:

$$
W^{\lambda \mu}=W_{1}\left(-g^{\lambda \mu}+\frac{q^{\lambda} q^{\mu}}{q^{2}}\right)+\frac{W_{2}}{m_{A}^{2}}\left(p^{\lambda}-\frac{(p q)}{q^{2}} q^{\lambda}\right)\left(p^{\mu}-\frac{(p q)}{q^{2}} q^{\mu}\right)
$$

where $m_{A}$ is the target mass.

With Eq. (1.32) and Eq. (1.32), Eq. (1.31) becomes

$$
\frac{d^{2} \sigma}{d \Omega_{e^{\prime}} d E_{e^{\prime}}}=\left(\frac{d \sigma}{d \Omega_{e^{\prime}}}\right)_{\mathrm{M}}\left[W_{2}(|\mathbf{q}|, \omega)+\mathbf{2} \mathbf{W}_{\mathbf{1}}(|\mathbf{q}|, \omega) \tan ^{2} \frac{\theta_{\mathbf{e}}}{\mathbf{2}}\right]
$$

where $\left(\frac{d \sigma}{d \Omega_{e^{\prime}}}\right)_{\mathrm{M}}$ is the Mott cross section

$$
\left(\frac{d \sigma}{d \Omega_{e^{\prime}}}\right)_{\mathrm{M}}=\frac{\alpha^{2} \cos ^{2}\left(\frac{\theta_{e}}{2}\right)}{4 E_{e}^{2} \sin ^{4}\left(\frac{\theta_{e}}{2}\right)}
$$

The virtual photon exchanged in electron scattering has both longitudinal and transverse polarizations. Thus we can also rewrite the cross section in terms of the longitudinal and transverse structure functions, $R_{L}$ and $R_{T}$ : 


$$
\begin{aligned}
& \frac{d^{2} \sigma}{d \Omega_{e^{\prime}} d E_{e^{\prime}}}=\left(\frac{d \sigma}{d \Omega_{e^{\prime}}}\right)_{\mathrm{M}} {\left[\left(\frac{Q^{2}}{|\mathbf{q}|^{2}}\right)^{2} R_{L}(|\mathbf{q}|, \omega)\right.} \\
&\left.+\left(\frac{1}{2} \frac{Q^{2}}{|\mathbf{q}|^{2}}+\tan ^{2} \frac{\theta}{2}\right) R_{T}(|\mathbf{q}|, \omega)\right] .
\end{aligned}
$$

\subsubsection{Exclusive cross section $\left(\mathrm{e}, \mathrm{e}^{\prime} \mathrm{p}\right)$}

In the exclusive (e,e'p) electron scattering experiment, the scattered electron and the knocked out proton are both detected using simultaneously two spectrometers, one dedicated to identify electrons and the other optimized for the proton identification.

Figure 1.8 shows a schematic of the $\left(\mathrm{e}, \mathrm{e}^{\prime} \mathrm{p}\right)$ reaction, where $k_{i}=\left(E_{i}, \mathbf{k}_{i}\right)$ and $k_{f}=\left(E_{f}, \mathbf{k}_{f}\right)$ are, respectively, the initial and the final four momenta of the incoming beam electron. $p_{p}=\left(E_{p}, \mathbf{p}_{p}\right)$ is the 4 momentum of the scattered proton, $\mathbf{k}_{i}, \mathbf{k}_{f}$ and $\mathbf{p}_{p}$ are measured directly by the experiments. Since electrons are light and their mass can be neglected, $E_{i} \approx\left|\mathbf{k}_{i}\right|$ and $E_{f} \approx\left|\mathbf{k}_{f}\right| . \quad E_{p}$ could be determined from $E_{p}=\sqrt{M_{p}^{2}+\mathbf{p}_{p}^{2}}$, where $M_{p}$ is the rest mass of proton. The 4-momentum transfer is defined by $q=k_{i}-k_{f}=(\omega, \mathbf{q})$.

The invariant cross section is given by [12]

$$
d \sigma=\frac{1}{(2 \pi)^{3}} \frac{E_{f}}{E_{i}} \frac{\alpha^{2}}{Q^{4}} \eta_{\mu \nu} W_{\mu \nu} d E_{f} d \Omega_{e} d^{3} \mathbf{p}_{p}
$$

where $d \Omega_{e}$ is the solid angle that covers the momentum of the electron in the laboratory, $\eta_{\mu \nu}$ and $W_{\mu \nu}$ are the electron and nuclear response tensors, respectively. With

$$
d^{3} \mathbf{p}_{p}=E_{p} p_{p} d E_{p} d \Omega_{p}
$$




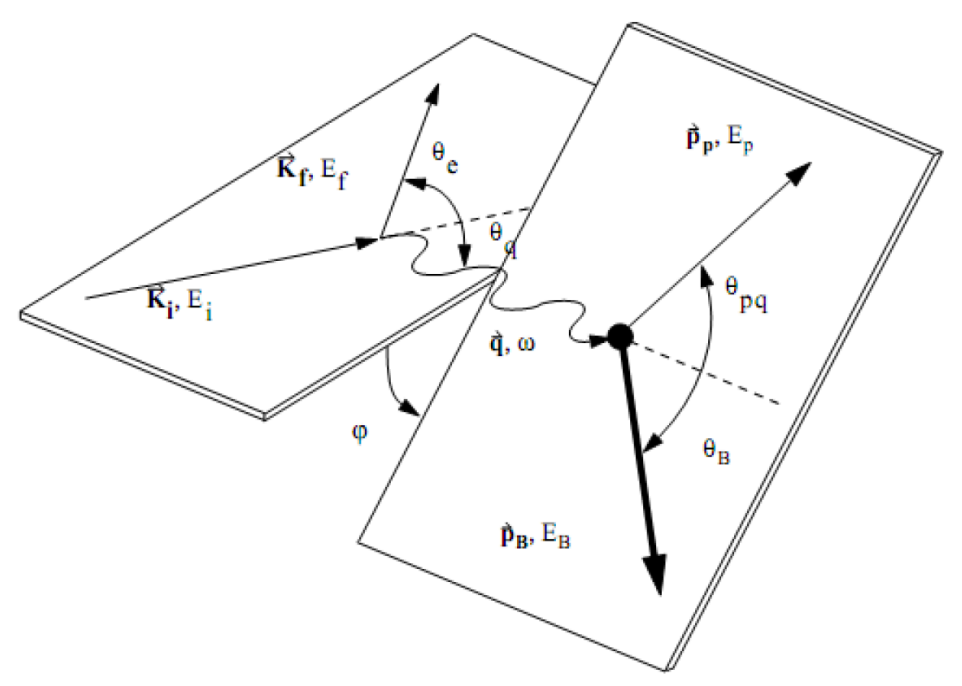

Figure 1.8: A diagram of the $\left(\mathrm{e}, \mathrm{e}^{\prime} \mathrm{p}\right)$ reaction

where $d \Omega_{p}$ describes the solid angle covers the proton momentum in the laboratory, and we can write the six fold differential cross section as below:

$$
\frac{d^{6} \sigma}{d E_{f} d \Omega_{e} d E_{p} d \Omega_{p}}=\frac{E_{p} p_{p}}{(2 \pi)^{3}} \frac{E_{f}}{E_{i}} \frac{\alpha^{2}}{Q^{4}} \eta_{\mu \nu} W_{\mu \nu} .
$$

For ultra-relativistic electrons the electron response tensor $\eta_{\mu \nu}$ becomes

$$
\eta_{\mu \nu}=2\left(k_{i \mu} k_{f \nu}+k_{f \mu} k_{i \nu}-k_{i} k_{f} g_{\mu \nu}\right)
$$

or

$$
\eta_{\mu \nu}=K_{\mu} K_{\nu}-q_{\mu} q_{\nu}-Q^{2} g_{\mu \nu}
$$

where $K_{\mu}=k_{i \mu}+k_{f \mu}$, and $q_{\mu}=k_{i \mu}-k_{f \mu}$.

The nuclear response tensor is the bi-linear products of the nuclear current operator matrix elements: 


$$
W_{\mu \nu}=\left\langle J_{\mu} J_{\nu}^{\dagger}\right\rangle
$$

where $\langle.$.$\rangle denotes averaging over initial states and summed over final states.$

Using the nuclear current conservation constraint, and the continuity requirement, we obtain

$$
\begin{array}{r}
q_{\mu} W^{\mu \nu}=W^{\mu \nu} q_{\nu}=0 \\
J_{z}=\frac{\omega}{\vec{q}} \rho
\end{array}
$$

After some math derivation, we can determine the form of the contraction between the electron and nuclear tensors:

$$
\eta_{\mu \nu} W_{\mu \nu}=4 E_{i} E_{f} \cos ^{2} \frac{\theta_{e}}{2}\left[V_{L} R_{L}+V_{T} R_{T}+V_{L T} R_{L T} \cos \phi+V_{T T} R_{T T} \cos 2 \phi\right] .
$$

The kinematic factors are expressed as

$$
\begin{aligned}
V_{L} & =\frac{Q^{4}}{\vec{q}^{4}} \\
V_{T} & =\frac{Q^{2}}{2 \vec{q}^{2}}+\tan ^{2}\left(\frac{\theta_{e}}{2}\right) \\
V_{L T} & =\frac{Q^{2}}{\vec{q}^{2}}+\left[\frac{Q^{2}}{\vec{q}^{2}}+\tan ^{2}\left(\frac{\theta_{e}}{2}\right)\right]^{\frac{1}{2}} \\
V_{T T} & =\frac{Q^{2}}{2 \vec{q}^{2}},
\end{aligned}
$$


the response functions can be written in terms of nuclear current tensor

$$
\begin{aligned}
R_{L} & =\left\langle W_{00}\right\rangle=\left\langle\rho \rho^{\dagger}\right\rangle \\
R_{T} & =\left\langle W_{x x}+W_{y y}\right\rangle=\left\langle J_{\|} J_{\|}^{\dagger}+J_{\perp} J_{\perp}^{\dagger}\right\rangle \\
R_{L T} \cos \phi & =-\left\langle W_{0 x}+W_{x 0}\right\rangle=-\left\langle\rho J_{\|}^{\dagger}+J_{\|} \rho^{\dagger}\right\rangle \\
R_{T T} \cos 2 \phi & =\left\langle W_{x x}-W_{y y}\right\rangle=\left\langle J_{\|} J_{\|}^{\dagger}-J_{\perp} J_{\perp}^{\dagger}\right\rangle,
\end{aligned}
$$

where $\rho$ is the charge component of the nuclear current.

$J_{\|}$and $J_{\perp}$ are the nuclear current's transverse components, and $R_{L}, R_{T}, R_{T L}$, and $R_{T T}$ are the response functions of kinematic variables which can obtained from the (e,e'p) reaction. $J_{\|}$and $J_{\perp}$ are respectively inside the scattering plane and orthogonal to the scattering plane, while both are also orthogonal to $\mathbf{q}$.

$R_{L}$ arises from the charge and longitudinal component of the nuclear current, $R_{T}$ is the incoherent sum of the contributions from the two transverse components of the nuclear current. $R_{T L}$ is, instead, the interference of the transverse component with the longitudinal component of the nuclear current in the scattering plane, while $R_{T T}$ is the interference between the two transverse components of the nuclear current.

In the special case of parallel kinematics, $\left(\mathbf{p}_{p} \| \mathbf{q}\right)$ only $R_{T}$ and $R_{L}$ contribute to the cross section expression.

\subsubsection{PWIA - Plane Wave Impulse Approximation}

In the Plane Wave Impulse Approximation(PWIA) [47], the virtual photon is totally absorbed by the proton, and the outcome proton could be considered as a free particle, as in Fig. 1.9. 


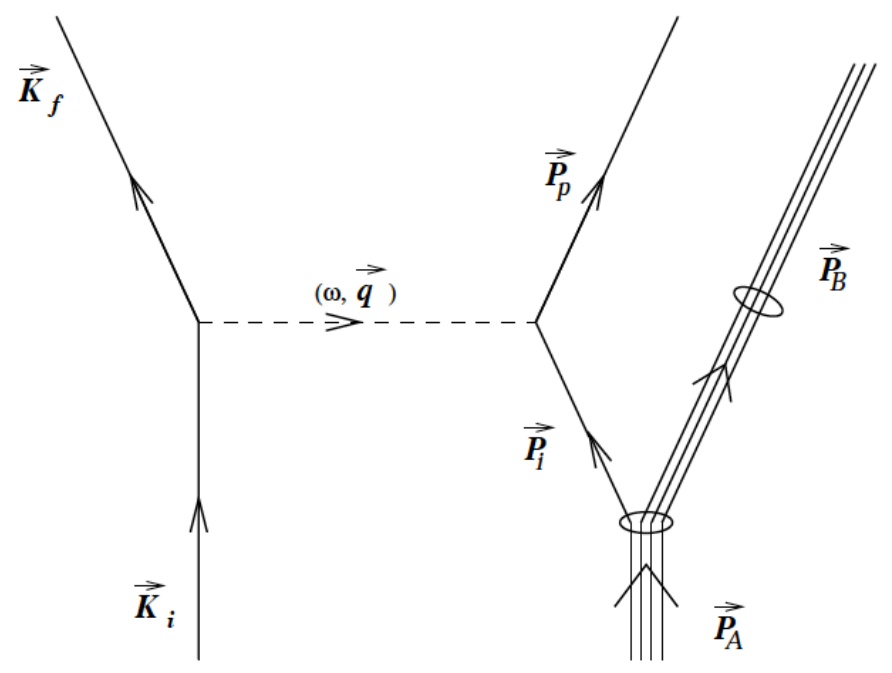

Figure 1.9: A diagram of the PWIA in (e,e'p) reaction [13].

In PWIA the missing momentum $\mathbf{p}_{\text {miss }}$, and the missing energy $E_{\text {miss }}$ are the important quantities, the missing energy is defined by

$$
E_{\text {miss }}=\omega-T_{p}-T_{B}
$$

where $T_{p}$ and $T_{B}$ are the kinetic energies of the scattered proton and the recoil nucleus, respectively. $T_{p}$ and $T_{B}$ can also be written as

$$
\begin{gathered}
T_{p}=\sqrt{\mathbf{p}_{\mathbf{p}}{ }^{2}+m_{p}^{2}}-m_{p} \\
T_{B}=\sqrt{\mathbf{p}_{\mathbf{B}}{ }^{2}+M_{B}^{2}}-M_{B} .
\end{gathered}
$$

Using the momentum conservation, the missing momentum is then 


$$
\mathbf{p}_{\text {miss }}=\mathbf{p}_{p}-\mathbf{q}=-\mathbf{p}_{B} .
$$

and using the energy conservation

$$
\begin{gathered}
E_{i}+M_{A}=E_{f}+E_{P}+E_{B} \\
\omega=E_{p}+E_{B}-M_{A}=\left(m_{p}+T_{p}\right)+\left(M_{B}+T_{B}\right)-M_{A}
\end{gathered}
$$

Merging Eq. (1.53) into Eq. (1.48), we get

$$
E_{\text {miss }}=m_{p}-M_{A}+M_{B},
$$

In Eq. (1.51) the missing momentum is the initial momentum of the proton inside the nucleus, and in Eq. (1.54) the missing energy describes the difference between the initial and final state in terms of nuclear binding energy.

Rewriting $M_{B}$ as

$$
\begin{aligned}
M_{B} & =\sqrt{E_{B}^{2}-\mathbf{p}_{B}^{2}} \\
& =\sqrt{E_{B}^{2}-\mathbf{p}_{\text {miss }}^{2}} \\
& =\sqrt{\left(\omega+M_{A}-E_{p}\right)^{2}-\mathbf{p}_{\text {miss }}^{2}}
\end{aligned}
$$

Then the missing energy becomes

$$
E_{m i s s}=m_{p}-M_{A}+\sqrt{\left(\omega+M_{A}-E_{p}\right)^{2}-\mathbf{p}_{m i s s}^{2}} .
$$




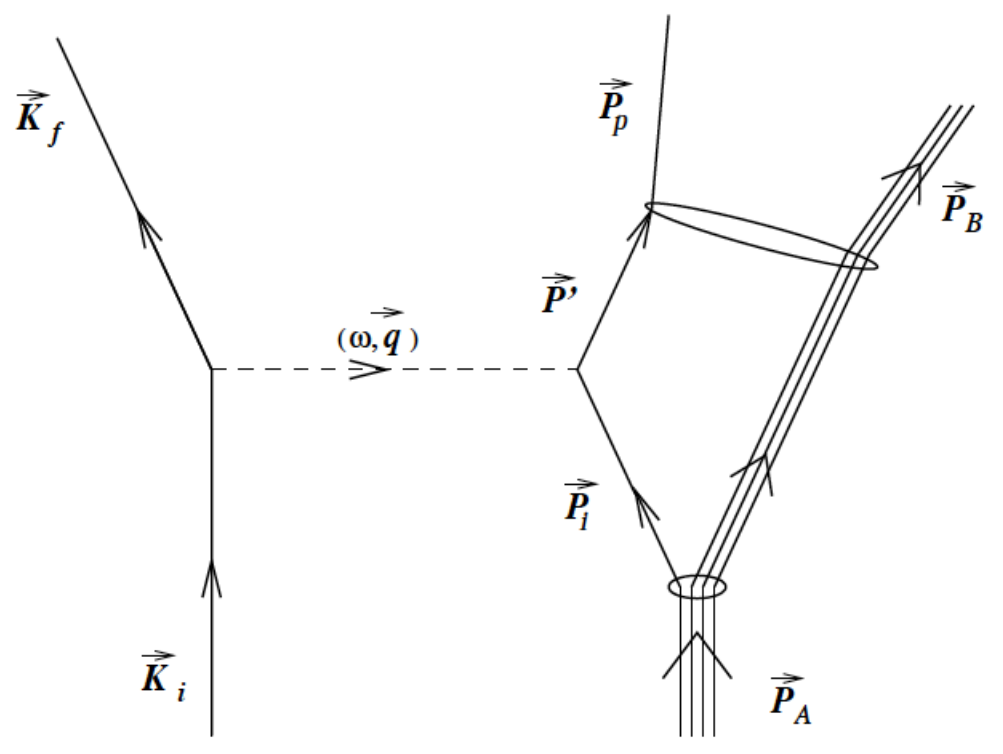

Figure 1.10: A diagram of the DWIA in (e,e'p) reaction [13].

In non-relativistic PWIA, we are able to factorize the cross section [47] as the following:

$$
\frac{d \sigma}{d E_{f} d \Omega_{e} d E_{p} d \Omega_{p}}=\frac{E_{p} p_{p}}{(2 \pi)^{3}} \sigma_{e p} S\left(E_{m i s s}, \mathbf{p}_{m i s s}\right)
$$

where $\sigma_{e p}$ is the off-shell cross section [95] and $S$ is the spectral function, which is the probability of finding a proton having momentum $\mathbf{p}_{\text {miss }}$ and binding energy $E_{m i s s}$ in the nucleus.

\subsubsection{DWIA - Distorted Wave Impulse Approximation}

In PWIA, the Final State Interaction (FSI) between the outgoing nucleon is considered as a free particle, and correlations with the remaining $A-1$ system are not actually taken into account. The Distorted Wave Impulse Approximation (DWIA) includes those correlations.

Figure 1.10 shows the diagram of the DWIA. 
The $\left(e, e^{\prime} p\right)$ cross section is then obtained by replacing the spectral function by a distorted spectral function $S^{D}$,

$$
\frac{d \sigma}{d E_{f} d \Omega_{e} d E_{p} d \Omega_{p}}=\frac{E_{p} p_{p}}{(2 \pi)^{3}} \sigma_{e p} S^{D}\left(E_{m i s s}, \mathbf{p}_{m i s s}, \mathbf{p}_{p}\right)
$$

The $E_{\text {miss }}$ and $\mathbf{p}_{\text {miss }}$ as well as $S^{D}$ depend on the proton momentum $\mathbf{p}_{p}$, since the FSI are depends on the particles/nucleons momenta.

FSI effects depend on kinematics: they will result in (1) a slight shift of the PWIA momentum distribution towards smaller values $(\sim 2-5 \mathrm{MeV}),(2)$ a reduction of the strength of the PWIA cross section between $30 \%$ and $80 \%$ depending on the specific orbital from which the electron is extracted, and (3) a broadening the missing energy spectrum [14]. 


\section{Chapter 2}

\section{Experimental Details}

The E12-14-012 experiment was performed at Hall A of the Thomas Jefferson National Accelerator Facility (Jefferson Lab or JLab), located in Newport News, Virginia. A total of 217.6 hours of scattering data was collected over a period of about three weeks from February to March of 2017. The dataset consists of 213 hours of exclusive (e,e'p) data acquired using five different kinematical settings, and 4.6 hours of inclusive (e,e') scattering taken using one kinematical setting.

Simulated Monte Carlo data used in analysis was generated using SIMC, a software package developed for Jefferson Lab's Hall C and Hall A. This chapter provides an overview of the experimental setup, the data collection, and the simulation methods used.

\subsection{Hall A Overview}

The primary features of Jefferson Lab Hall A are the two high-resolution spectrometers (HRSs). Each HRS is outfitted with similar detector packages developed to observe the products of electron scattering interaction. In our experiment, the left-hand spectrometer detected the scattered electrons and the right-hand spectrometer detected the protons knocked out from the target in the (e,e'p) reaction. For the inclusive reaction we used just the left arm spectrometer. The incident electron beam is supplied by the Continuous Electron Beam Accelerator Facility (CEBAF) at Jefferson Lab. 


\subsection{Beam}

\subsubsection{Accelerator}

The electron beam produced by CEBAF is first accelerated to $45 \mathrm{MeV}$ by an injector, and further acceleration is achieved using two 80-meter-long linear accelerators (linacs). The linacs were designed for an acceleration of $5 \mathrm{MeV} / \mathrm{m}$, to give a maximum energy of $4 \mathrm{GeV}$, but now are capable of over $7 \mathrm{MeV} / \mathrm{m}$, with a maximum electron energy of $5.7 \mathrm{GeV} /$ each. This was the result of an upgrade that lasted several years and was completed recently, opening the JLab $12 \mathrm{GeV}$ research era. The electrons can be recirculated around the accelerator loop, visible in Fig. 2.1, for maximum of four turns before being sent to the experimental Halls A, $\mathrm{B}$, and $\mathrm{C}$. The beam can be extracted at the end of each turn enabling each experimental halls to receive a range of electron beam energy [15]. The bends at each end are designed for a different pass around the accelerator, since the beam will be at a different energy each time. By sending some portion of the beam to an experimental hall and some portion for additional passes, it is possible to have the different halls operating with different beam energies at the same time without loss of beam time or intensity [16].

\subsubsection{Beam Position Measurement}

The position and direction of the beam are determined by two Beam Position Monitors (BPMs) located at distances of $1.286 \mathrm{~m}$ and $7.524 \mathrm{~m}$ upstream of the target. For currents above $1 \mu \mathrm{A}$, the position of the beam inside each BPM is determined to within $100 \mu \mathrm{m}$. Each BPM contains an assembly of four wire antennas, in two opposing pairs, and as beam passes between them, it induces a signal in each wire. This setup is shown in Fig. 2.2. The strength of this current in a given wire is proportional to its distance from the beam, and thus 


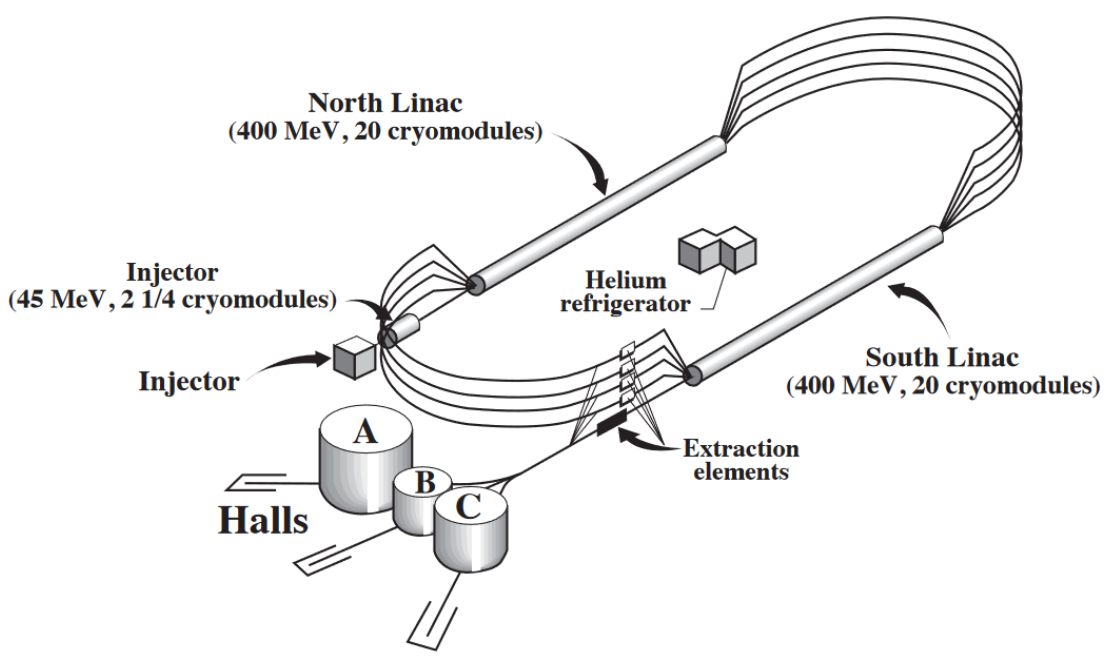

Figure 2.1: Schematic of the accelerator facility, CEBAF. The beam is produced at the injector, and two superconducting linacs are designed to accelerate it up to $4 \mathrm{GeV}$ before it is sent to Halls A, B, and C.

with four wires the position of the beam within the BPM cavity can be pinpointed. Each BPM also has an associated wire scanner called a super-harp which is used to calibrate the absolute position of the BPM with respect to the Hall A coordinate system. The super-harps themselves are regularly surveyed and are reliable to within $200 \mu \mathrm{m}$. [15]
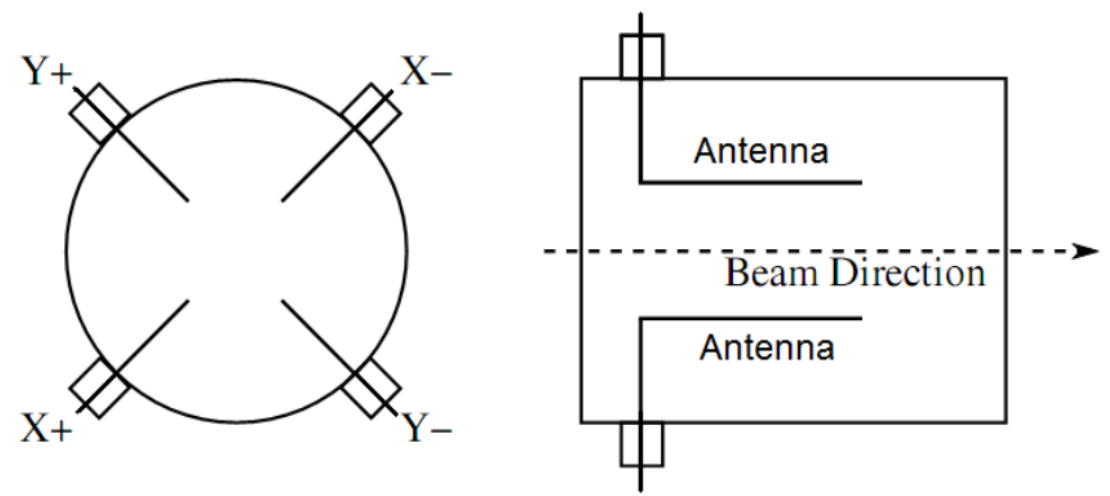

Figure 2.2: Schematic of the beam position monitor system [16]. 


\subsubsection{Beam Current Measurement}

The Beam Current Monitor (BCM) system consists of several parts. The absolute reference is measured by a parametric current transformer called an Unser monitor, as described in Ref. [17]. However, the Unser monitor's output drifts on a timescale of minutes, and for this reason, it is not used to continuously monitor beam current, but rather to calibrate the other parts of the BCM. There are two resonant RF cavities contained in the same temperature-controlled, magnetically-shielded housing as the Unser monitor (one upstream and one downstream), and these are used for the continuous current monitoring. The cavities are cylindrical waveguides which are tuned to the frequency of the beam and produce an output voltage proportional to the beam current. The signal from each of these cavities is split into two parts - one which is sampled and one which is integrated. Sampled data is collected every second and records the average current of the beam during that second, while

integrated data accumulates over the course of a run and represents the total beam charge for that run. These BCMs are calibrated using the Unser monitor every several months and provide current measurements to within $0.5 \%$ for currents down to $1 \mu \mathrm{A}$. [15].

\subsubsection{Beam Energy Measurement}

There are two ways in which the energy of the electron beam is measured [15]. The first of these, the Arc method, is so called because it takes place in the Arc section of the beam-line, where the beam is directed into Hall A from the main accelerator ring using eight dipole magnets. By measuring the bending angle $\theta$ of the beam and calculating the magnetic field integral $\int \vec{B} \cdot \overrightarrow{d l}$ of the dipole magnets it passes through, the momentum of the beam can be calculated as

$$
p=k \frac{\int \vec{B} \cdot \overrightarrow{d l}}{\theta}
$$


The nominal bending angle of the beam is $34.3^{\circ}$, and the actual angle is measured using two pairs of super-harps placed on either side of the Arc section. The field of the dipole magnets is determined using a ninth dipole which serves as a reference. The systematic uncertainty $(\Delta E / E)$ in this measurement is on the order of $10^{-4}$.

The other method to measure the beam energy is the $e P$ method, which utilizes the ${ }^{1} \mathrm{H}\left(\mathrm{e}, \mathrm{e}^{\prime} \mathrm{p}\right)$ elastic scattering reaction [15]. This measurement is performed by a device about $17 \mathrm{~m}$ upstream of the target, show in Fig. 2.3. Incident electrons are scattered off a polyethylene $\left(\mathrm{CH}_{2}\right)$ target, and the angle of the scattered electron $\left(\theta_{e}\right)$ and the recoil proton $\left(\theta_{p}\right)$, and the energy of the incident electron is calculated as

$$
E=M_{p} \frac{\cos \left(\theta_{e}\right)+\sin \left(\theta_{e}\right) / \tan \left(\theta_{p}\right)-1}{1-\cos \left(\theta_{p}\right)}+O\left(m_{e}^{2} / E^{2}\right)
$$

, where $M_{p}$ and $m_{e}$ are the proton and electron masses respectively. The reaction products are detected by silicon-strip detectors - one for protons and eight for electrons corresponding to different regions of beam energy. The device has two arms, symmetric across the beam axis, which serves to cancel uncertainty in the beam position and direction to first order. The systematic uncertainty $(\Delta E / E)$ of this method is comparable to that of the Arc method, and measurements by the two methods agree well within that uncertainty.

\subsection{HRS - High Resolution Spectrometers}

Hall A is instrumented with two High Resolution Spectrometers (HRSs), which are used to detect the outgoing protons and electrons in this experiment. The spectrometers operate over a momentum range of $0.8-4.0 \mathrm{GeV} / \mathrm{c}$, with a resolution $(\mathrm{dp} / \mathrm{p})$ on the order of $10^{-4}$. Each spectrometer has three superconducting quadrupole magnets and one non-superconducting 


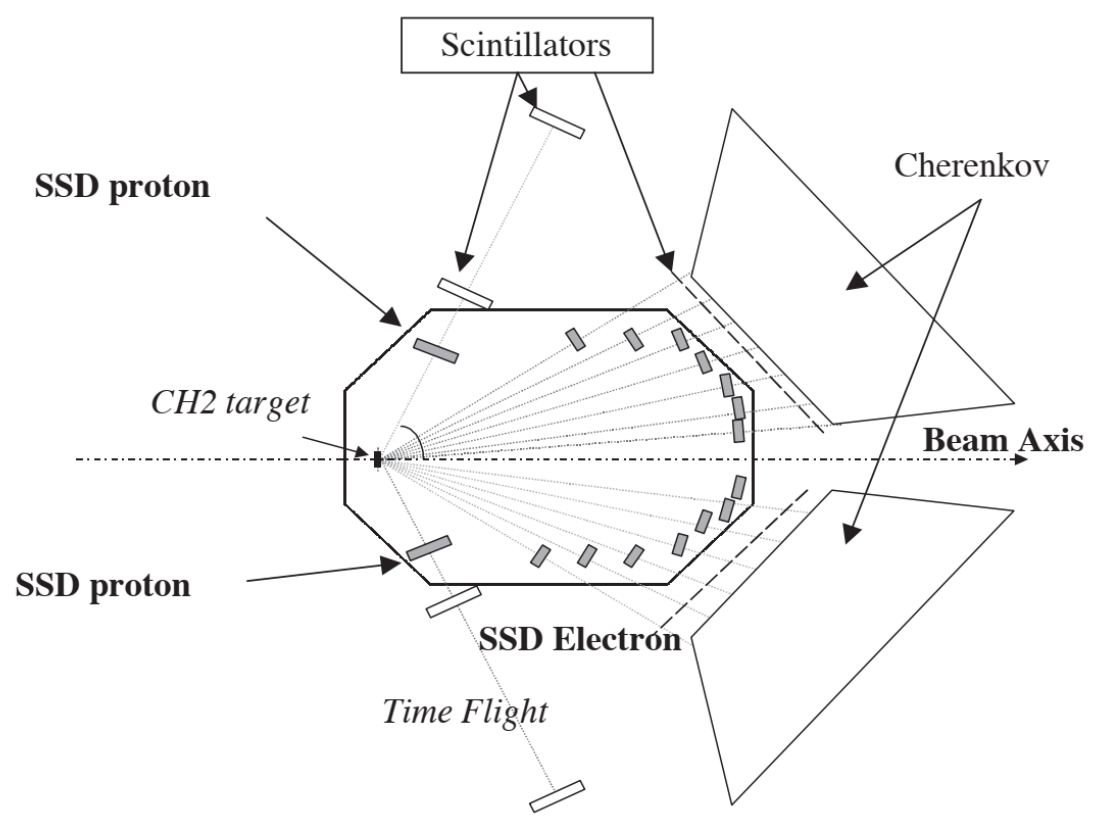

Figure 2.3: Schematic of the $e P$ energy measurement device [15].

dipole magnet, arranged as QQDQ, used for focusing and directing charged particles. The main properties of the spectrometers are listed in Tab. 2.1 [15].

\subsection{Detector Package}

\subsubsection{Vertical Drift Chambers}

Both the left and right HRS contain an identical pair of vertical drift chambers (VDCs) which are used to determine the trajectories of charged particles entering the spectrometer. These chambers are horizontal in the lab's reference frame and parallel to each other. Each VDC contains two wire planes, orthogonal to each other and at a $45^{\circ}$ angle to the dispersive and non-dispersive directions of the spectrometer, as shown in Figure (FIG). Each wire plane contains 368 wires, spaced $4.24 \mathrm{~mm}$ apart. The first VDC in each spectrometer is located as close to the focal plane as possible, and the other is placed $0.335 \mathrm{~m}$ above it. Inside 
Table 2.1: Hall A HRS - High resolution Spectrometer acceptances and detailed resolutions

\begin{tabular}{|c|c|}
\hline Bending angle & $45^{\circ}$ \\
\hline Optical length & $23.4 \mathrm{~m}$ \\
\hline Momentum range & $0.3-4.0 \mathrm{GeV} / \mathrm{c}$ \\
\hline Momentum acceptance & $-4.5 \%<\delta p / p<+4.5 \%$ \\
\hline Momentum resolution & $1 \mathrm{e}-4$ \\
\hline Dispersion at the focus (D) & $12.4 \mathrm{~m}$ \\
\hline Radial linear magnification $(\mathrm{M})$ & -2.5 \\
\hline D/M & 5.0 \\
\hline Angular range (HRS-L) & $12.5-150^{\circ}$ \\
\hline Angular range (HRS-R) & $12.5-130^{\circ}$ \\
\hline Angular acceptance (horizontal) & $\pm 30 \mathrm{mrad}$ \\
\hline Angular acceptance (vertical) & $\pm 60 \mathrm{mrad}$ \\
\hline Angular resolution (horizontal) & $0.5 \mathrm{mrad}$ \\
\hline Angular resolution (vertical) & $1.0 \mathrm{mrad}$ \\
\hline Solid angle at $\delta p / p=0, y_{0}=0$ & $6 \mathrm{msr}$ \\
\hline Transverse length acceptance & $\pm 5 \mathrm{~cm}$ \\
\hline Transverse position resolution & $1 \mathrm{~mm}$ \\
\hline
\end{tabular}
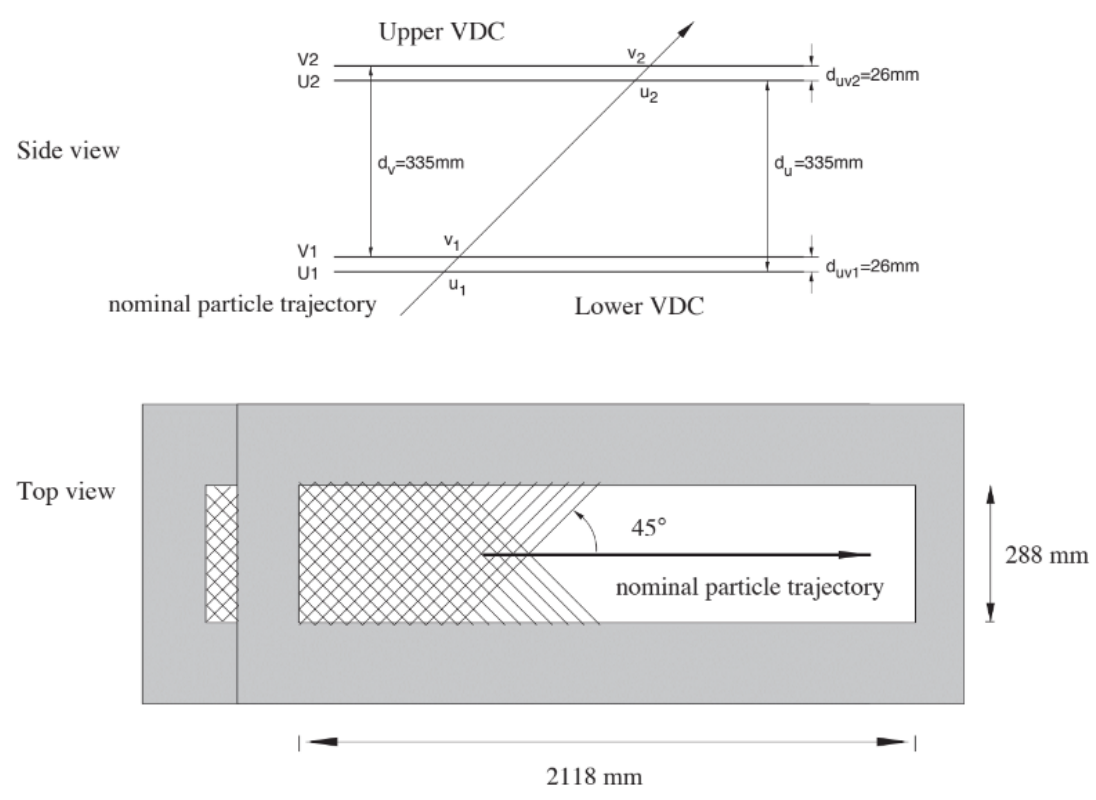

Figure 2.4: Schematic of the HRS Vertical Drift Chambers [15].

each VDC is a mixture of gases - $62 \%$ argon and $38 \%$ ethane - which ionize when a charged particle passes through, and then drift in the strong electric field created between gold-plated 
Mylar planes, to create a signal on the wire arrays. A time-to-drift digital converter (TDC) measures the time for an ionized electron to reach the detector wire, which is used to find the perpendicular distance from the particle's trajectory to the wire. Each event produces a signal on an average of 5-6 adjacent wire cells, and a linear interpolation of the distances to each wire is used to construct the trajectory. In the focal plane, the position resolution is $\sigma_{x / y} \approx 100 \mu \mathrm{m}$, and the angular resolution is $\sigma_{\theta / \phi} \approx 0.5 \operatorname{mrad}[15]$.

\subsubsection{Scintillators}

Each HRS contains also two planes of plastic scintillator, called S1 and S2, and can have a third (S0) added for experiments which require it. These planes are used to provide triggering information. S0 and S2 were used to form the triggers for this experiment, which will be described in more detail in a later section. $\mathrm{S} 2$ is composed of 6 overlapping $5 \mathrm{~mm}$ thick scintillator, each viewed by 2 PMTs, with a time resolution of about 0.3 ns [15].

\subsubsection{Gas Čerenkov detector}

One element of particle identification in the HRS is a gas Čerenkov detector, located between the two scintillator planes. The detector contains $\mathrm{CO}_{2}$ at atmospheric pressure, and observes the ring of Čerenkov light created when a particle passes through at high velocity. There are 10 spherical mirrors and 10 corresponding PMTs to detect the light, as shown in Fig. 2.5. There is a momentum threshold for a charged particle to produce this Čerenkov light, dependent on the medium's index of refraction and the mass of the particle. For this detector, that threshold is $0.017 \mathrm{GeV} / \mathrm{c}$ for electrons and $4.8 \mathrm{GeV} / \mathrm{c}$ for pions. Given the kinematics of the experiment, any pions produced will be below this threshold, and thus one can use the lack of Čerenkov light to eliminate any pion events. 


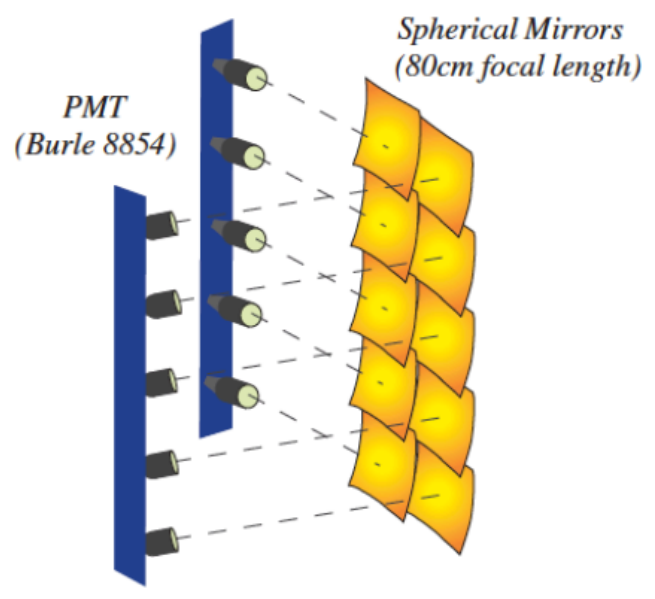

Figure 2.5: Schematic of the mirrors and PMTs inside the HRS Gas Čerenkov detector. [15].

\subsubsection{Lead Glass Calorimeters}

After the gas Čerenkov detector, both the left and right HRSs contain two layers of shower detectors. These detectors are composed of lead glass blocks viewed by PMTs, where electrons or protons can create a shower of high-energy photons via Bremsstrahlung. These photons in turn create electron-positron pairs, and the process repeats, creating a shower of photons which the PMTs observe. Due to their long interaction length, pions will not create a shower in the calorimeter, so it serves as another way to reject those events, as well as measuring the energy deposited by an electron or proton. In the HRS-L, the two lead-glass layers are each made of 34 blocks, each $15 \mathrm{~cm} \times 15 \mathrm{~cm} \times 30$ (or 35$) \mathrm{cm}$, and they lie perpendicular to the beam direction, as shown in Fig. 2.6. In the HRS-R, the first layer has 48 blocks, $10 \mathrm{~cm} \times 10 \mathrm{~cm} \times 35 \mathrm{~cm}$, perpendicular to the beam direction, and the second layer has 80 blocks, $15 \mathrm{~cm} \times 15 \mathrm{~cm} \times 35 \mathrm{~cm}$, oriented parallel to the beam direction. Due to the longer path length, this means that the HRS-R has a better resolution than that of the HRS-L[15]. 

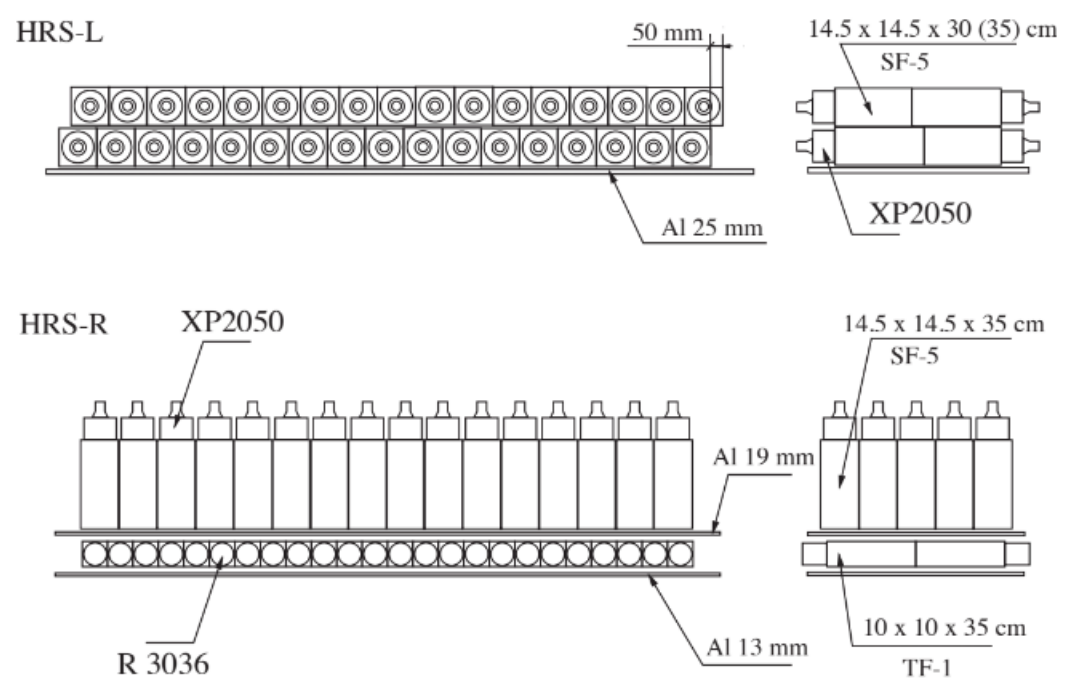

Figure 2.6: Representation of the lead glass calorimeters in the left (top) and right (bottom) HRS [15].

\subsection{Hall A E12-14-012 target system}

There were four different materials used in the targets during our experiment - argon, titanium, carbon, and aluminum. The argon target was a closed gas cell made of aluminum (7075 alloy). The experiment also had was a single-foil target for both titanium and carbon, an aluminum dummy target which was used to investigate backgrounds, and a carbon multifoil target (9 individual foils), used for tuning and testing of the HRS optics. Schematics of the target configuration is described in Ref. [18], and it is represented in Fig. 2.7, in which the gas cell, dummy, and optical targets are all visible.

The argon gas cell is the same cell that was used to hold tritium and helium gases during the MARATHON experiment which was also conducted in Hall A [19]. The cell is a $25 \mathrm{~cm}$ long cylinder oriented along the direction of the beam, with a diameter of $12.7 \mathrm{~mm}$ and a hemispherical rounded tip. The target walls are roughly $0.4-0.5 \mathrm{~mm}$ thick on the sides, and $0.25 \mathrm{~mm}$ at the entrance and exit windows. The cell was attached to the standard Hall A cryogenic system. The argon gas inside the cell was maintained at approximately $291 \mathrm{~K}$ and 


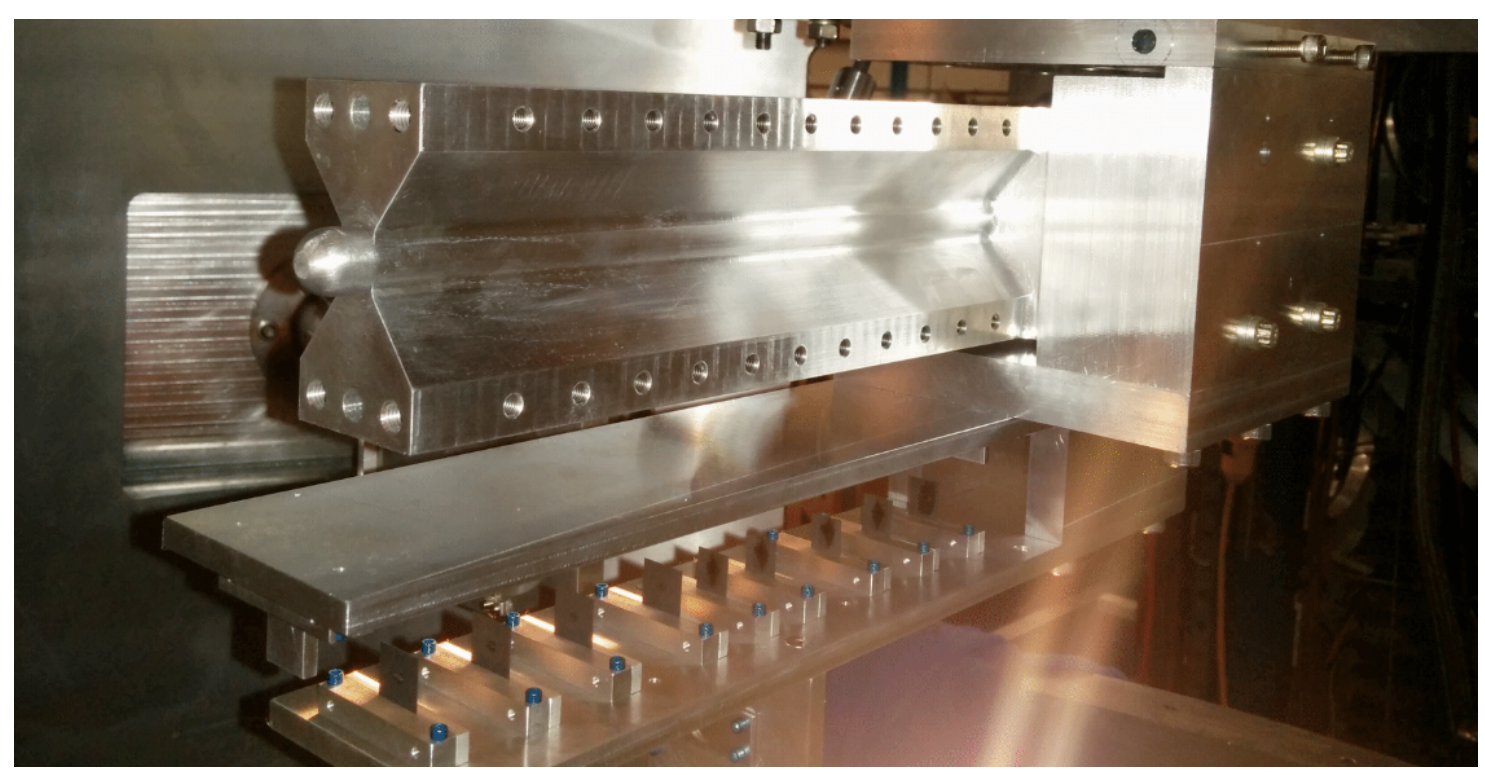

Figure 2.7: Photo showing the closed gas cell target (top), the aluminum dummy target (center), and the multi-foil carbon optical target (bottom) [20].

$3.45 \mathrm{MPa}$, resulting in a density of $58.2 \mathrm{~kg} / \mathrm{m}^{3}$ [20]. However, it was observed that while the beam was turned on, it deposits heat into the argon gas, resulting in a local expansion (and thus lower density) at the beam's location. The relationship between beam current and target gas density was analyzed and it described in Ref. [21].

To help in determining background created by the cell itself, data was also taken on an aluminum dummy target. This target consists of two blocks of aluminum, positioned $25 \mathrm{~cm}$ apart, to represent the entrance and exit of the gas cell. Each of these blocks had a thickness of $0.35 \mathrm{~g} / \mathrm{cm}^{2}$. In addition to finding the background, this target was used to calculate the inclusive $\mathrm{Al}\left(\mathrm{e}, \mathrm{e}^{\prime}\right)$ cross section.

The optical target consisted of a series of 9 carbon foils, each with a thickness of $0.35 \mathrm{~g} / \mathrm{cm}^{2}$. The foils were positioned at $\mathrm{z}=0, \pm 2, \pm 5, \pm 7.5$, and $\pm 10 \mathrm{~cm}[20]$, and were used to align the spectrometer optics.

Lastly, there were two simple foil targets, one carbon and one titanium. The carbon foil had 
a thickness of $0.17 \mathrm{~g} / \mathrm{cm}^{2}$, and was used to measure the inclusive cross section for comparison to existing carbon data, as a way to develop and verify the analysis procedure. The titanium foil had a thickness of $0.73 \mathrm{~g} / \mathrm{cm}^{2}$, and was used to conduct both inclusive and exclusive cross section measurements [20].

\subsection{Triggers and Data Acquisition}

There are several different components used to build the triggers that control data taking. Both the left and right spectrometers have two planes made of scintillator material (labeled S0 and S2) that can be used, and additionally one can build triggers using the signals from the gas Čerenkov detectors (GC), the left-arm pion rejector (PR), and the right arm preshower/shower (PS). Table 2.2 lists the configurations of these signals that are used by the DAQ.

Table 2.2: Trigger types and trigger logic configurations.

\begin{tabular}{|c|c|}
\hline T1 & $(\mathrm{S} 0 \& \& \mathrm{~S} 2) \& \&(\mathrm{GC}|| \mathrm{PR})[\mathrm{LEFT}]$ and $(\mathrm{S} 0 \& \& \mathrm{~S} 2)[\mathrm{RIGHT}]$ \\
\hline T2 & $(\mathrm{S} 0|| \mathrm{S} 2) \& \&(\mathrm{GC}|| \mathrm{PR})[\mathrm{LEFT}]$ and $(\mathrm{S} 0|| \mathrm{S} 2) \& \&$ NOT$\left(\mathrm{PS} \_\mathrm{e}\right)[\mathrm{RIGHT}]$ \\
\hline T3 & $(\mathrm{S} 0 \& \& \mathrm{~S} 2) \& \&(\mathrm{GC}|| \mathrm{PR})[\mathrm{LEFT}]$ \\
\hline T4 & $(\mathrm{S} 0 \& \& \mathrm{~S} 2)[\mathrm{RIGHT}]$ \\
\hline T5 & $(\mathrm{S} 0 \| \mathrm{S} 2) \& \&(\mathrm{GC}|| \mathrm{PR})[\mathrm{LEFT}]$ \\
\hline T6 & $(\mathrm{S} 0|| \mathrm{S} 2) \& \&$ NOT $\left(\mathrm{PS} \_\mathrm{e}\right)[\mathrm{RIGHT}]$ \\
\hline
\end{tabular}

The triggers that are interpreted as an event observation are T3 (which only includes the left spectrometer arm, for inclusive observations) and T1 (for exclusive observations involving both arms). The other triggers are used to provide a sample by which to calculate the trigger efficiency. For example, to calculate the trigger efficiency for the inclusive measurement, we take a sample consisting of single-track events that meet acceptance and particle-identification cuts which satisfy T5 (signal on either scintillator plane), and find the percentage of that sample that also satisfies T3 (signal on both scintillator planes). We 
follow a similar procedure for the exclusive measurement, using $\mathrm{T} 2$ and $\mathrm{T} 1$, respectively. Added complexity in this case results from the time-coincidence requirement involved in the exclusive case. We compare the efficiency of T1 to the product of the efficiencies of T3 and T4 - if the proton and electron observations from the same event were perfectly paired, these values would be the same. In summary we have the definitions as in Eqs. (2.1, 2.2 and 2.3).

$$
\begin{aligned}
& \operatorname{Eff}(T 1)=\frac{\# \text { Events passing } T 1}{\# \text { Events passing } T 2} \\
& \operatorname{Eff}(T 3)=\frac{\# \text { Events passing } T 3}{\# \text { Events passing } T 5} \\
& \operatorname{Eff}(T 4)=\frac{\# \text { Events passing } T 4}{\# \text { Events passing } T 6}
\end{aligned}
$$

Figure 2.8 represents the difference between $\mathrm{T} 1$ and $\mathrm{T} 3{ }^{*} \mathrm{~T} 4$ in kinematics 3 , and Fig. 2.9 shows the same efficiencies as function of the argon cell's length.

\subsection{Monte Carlo}

The Monte Carlo simulation for the E12-14-012 JLab experiment was performed using the SIMC software developed for JLab's Halls A and C. The MC is used primarily to correct the experimental data for the acceptance of the spectrometer, i.e. the probability that a given event will make it through and be detected, so that we can later determine the data yield. The Monte Carlo generates events uniformly in a phase space and then simulates their transport through the components of the detectors inside the HRS. SIMS also contains a subroutine (COSY Infinity) used to model the optical transport through the spectrometer 
Trig_eff Kin3

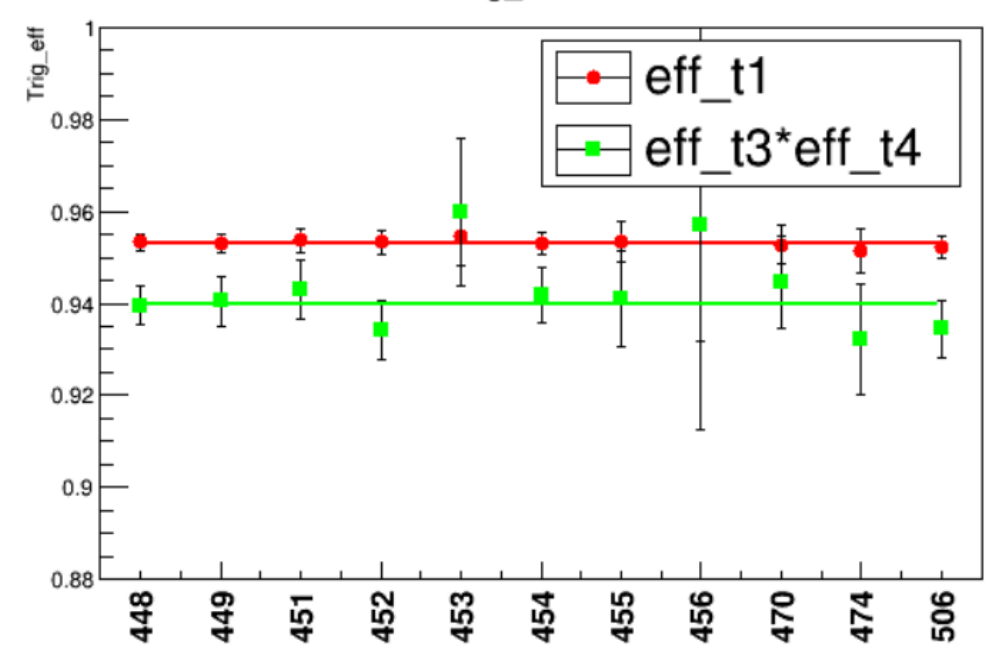

Figure 2.8: T1 efficiency as function of run number (red data points) and product of the T3 and T4 efficiency as function of run number. The red and green line are a fit with a polynomial of order zero to the data.
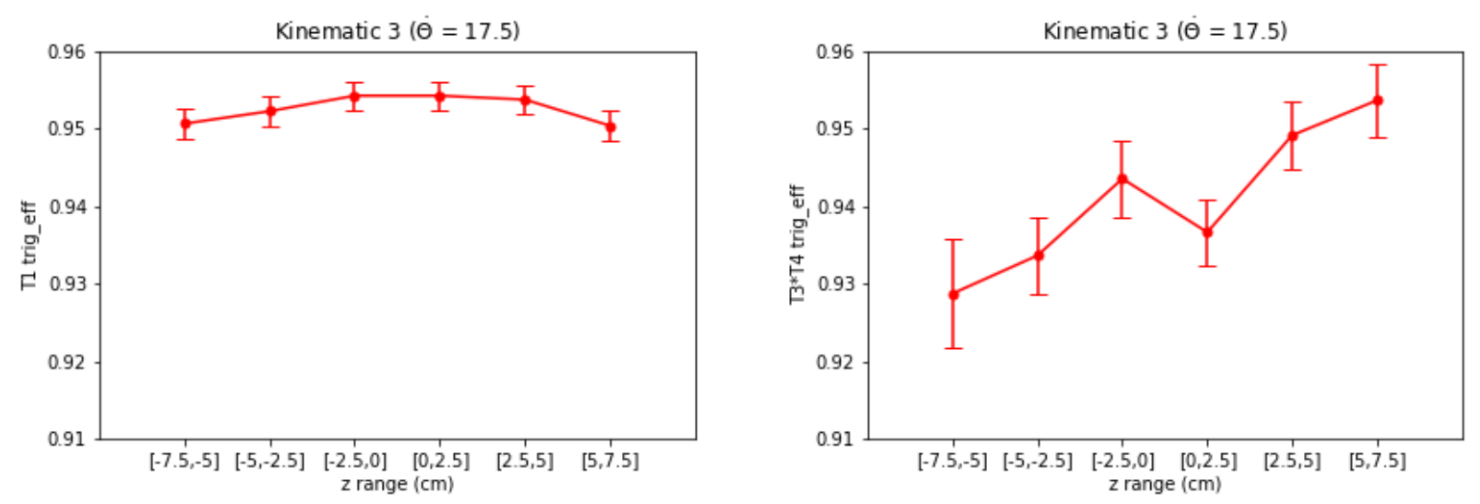

Figure 2.9: T1 and product T3*T4 efficiencies as function of the target length for the argon cell.

magnets. COSY generates an optical matrix that projects the events from the target to the focal plane of the spectrometers. COSY uses the HRS magnet positions, dimensions and field maps, to generates a forward matrix that projects rays at the target to the focal point. The position of each event is calculated for several points: beginning and end of each magnet as well as position $2 / 3$ of the way through the first two quadrupole magnets. The tracks in the focal plane are recorded for all particles which make it all the way through the detector 
stack.

SIMC is capable of simulating a variety of target nuclei, ranging from very light ones like hydrogen, to carbon, iron all the way up to lead. SIMS can readout for each nuclei correlation matrices and could use the spectral functions as input files. For each nuclei the distribution of protons or neutrons inside each orbitals should be provided together with transparency and the value for the fermi energy for that particular nuclei. We used approximate theoretical spectral functions for both Ar and Ti. There are no available data on Ar and Ti so the spectral function are derived as an extrapolation from lighter nuclei. The scope of our experiment is to measure spectral functions for both Ar and Ti. There are additional nuclear effects that are modeled in SIMC like radiative correction, Coulomb corrections and Energy Loss that will be described in the remaining of this section.

The goal for SIMC is to be able to compute a Monte Carlo yield and then correct it by the effects as they appear in the data, such as radiative and Coulomb correction, energy loss and spectrometer acceptance. If the experimental data are properly simulated, the yields from the Monte Carlo and the experimental data will be in agreement and from the ratio between data and $\mathrm{MC}$ the cross section could be extracted as function of the cross section model used as input to the MC:

$$
\frac{d^{2} \sigma}{d \Omega d E}=\frac{Y_{d a t a} \cdot C_{r a d}}{\Delta E^{\prime} \cdot \Delta \Omega \cdot N_{\text {Scatterers }}}
$$

where $Y_{\text {data }}$ is the charge normalized data/MC yield integrated over the acceptances of the spectrometers, $C_{\text {rad }}$ is the correction due to radiative and Coulomb effects, $\Delta E^{\prime} \cdot \Delta \Omega$ is the phase space, and $N_{\text {Scatterers }}$ is the number of scattering centers in the target. 


$$
\frac{\sigma_{d a t a}^{b o r n}}{\sigma_{M C}^{b o r n}}=\frac{Y_{d a t a}}{Y_{M C}}
$$

where $Y_{\text {data }}$ is the charge normalized data yield integrated over the acceptance of the spectrometer, $Y_{M C}$ is the Monte Carlo simulated yield, $\sigma_{b o r n}$ model is the Born cross section model, and $\sigma_{d a t a}^{b o r n}$ is the quantity we want to measure.

\subsubsection{Radiative Corrections}

The cross sections need to be corrected for effects from internal and external radiative processes. Internal radiative processes include vacuum polarization, vertex corrections, and internal bremsstrahlung. Electrons loose energy through bremsstrahlung while passing through the target material, which is an external effect as in Fig. 2.10 external bremsstrahlung.

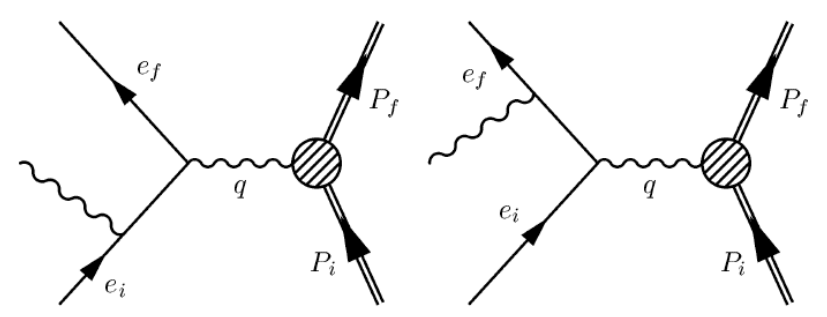

Figure 2.10: The incoming and/or scattered electron can lose energy through bremsstrahlung.

In our analysis, the radiative corrections were originally calculated with the the peaking approximation method of Mo and Tsai [22]. But, as was shown in previous JLab experiment, this approximation does not do a good job for thick targets at low $\mathrm{x}$ and the full 2-D integral needs to be calculated. We then adopted the approach as described in Ref. [23] and used to do radiative corrections for SLAC experiments. In this approach, a complete calculation of Mo and Tsai's formula for external effects is done. To determine the systematic uncertainty from the choice of the cross section model used to calculate the radiative correction, the 
model was rescaled in $\left(Q^{2}\right) / 2$ and the correction recalculated. A theoretical uncertainty of $1 \%$ was added as additional systematic error. The boiling effect alter the density of the gas target cell and affect the radiative corrections as well. For our experiment the radiative corrections were computed at different boiling point for the Ar gas target and the variations added in quadrature and considered as additional systematic errors.

\subsubsection{Coulomb Correction}

The MC Yield should also be corrected for the Coulomb distortion of the electron wave function by the electrostatic field of the nucleus. The nucleus has two effects on the electron: it is accelerated due to the attractive force when in close proximity to the nucleus, increasing the electron's momentum and also, the attractive force focuses the electron wave function. A distorted wave Born approximation (DWBA) calculation using Dirac wave functions is the correct approach [24] but because it is too computationally intensive, a local Effective Momentum Approximation (EMA) is used [25].

\subsubsection{Energy Loss}

Electrons loose energy through interactions as the come and they go out of the nuclear target. The energy of the incoming electron can be lower than the measured beam energy at the vertex, and the measured energy of the detected electron can be lower than its energy at the vertex. The calculated physics quantities in the analysis engine has to be corrected for the energy loss. The energy loss is the result of the charged particle interacting with matter. The incoming electron loses energy in the target material, the target cell walls and the target cell entry windows as it traverses the target cell. The outgoing scattered electron passes through the remaining of the target, which for solid targets depends on the scattering 
angle, then the target-chamber exit window, the air gap between the target system and the spectrometer magnets and finally, the entrance window to the spectrometer. The energy loss is calculated by using the Bethe-Bloch equation [26], and the energy loss is parametrized in terms of momentum transfer with the assumption that the scattering event takes place in the center of the target.

\subsubsection{Target model}

The existing Monte Carlo code contained prescriptions for the foil targets, as well as cylindrical targets oriented horizontally and vertically. However, to ensure the most accurate cross section results, we have developed and implemented in SIMC a precise geometrical representation of our argon cell target. The target is an aluminum cell, mostly cylindrical with a rounded tip, as can be seen in Fig. 2.11.

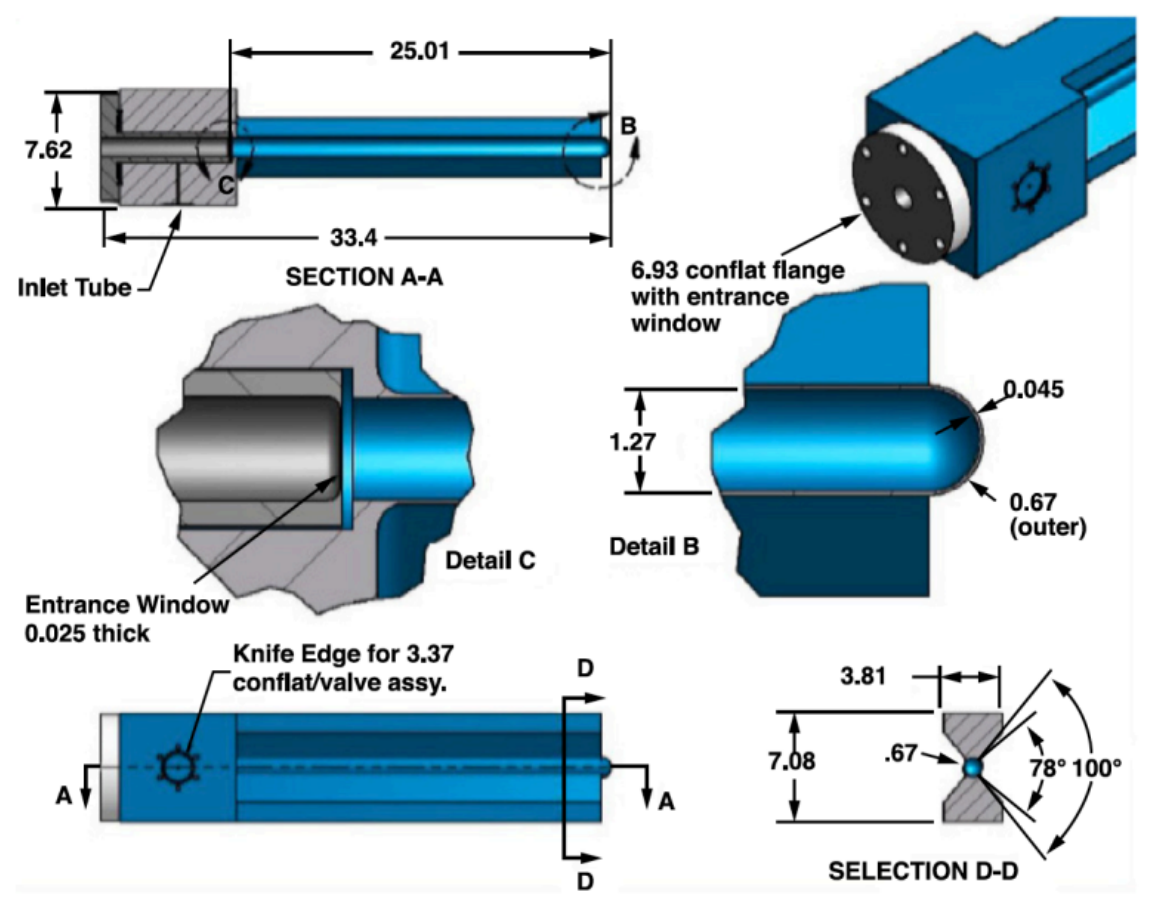

Figure 2.11: Schematic of our argon gas target cell. 


\subsection{Data Collection}

The analysis presented in this thesis uses data collected with the settings as in Tab. 2.3. The only difference of data collection setting for ${ }^{48} \mathrm{Ti}$ target is the incident electron energy.

Table 2.3: Kinematics settings used to collect the data analyzed here.

\begin{tabular}{|ccccccccc|}
\hline & $E^{\prime}$ & $\theta_{e}$ & $P_{p}$ & $T_{p}$ & $\theta_{p}$ & $|\mathbf{q}|$ & $p_{m}$ & $E_{m}$ \\
\hline & $(\mathrm{MeV})$ & $(\mathrm{deg})$ & $(\mathrm{MeV} / c)$ & $(\mathrm{MeV})$ & $(\mathrm{deg})$ & $(\mathrm{MeV} / c)$ & $(\mathrm{MeV} / \mathrm{c})$ & $(\mathrm{MeV})$ \\
\hline $\mathrm{Ar}$ & 1,777 & 21.5 & 915 & 372 & -50.0 & 865 & 50 & 73 \\
\hline $\mathrm{Ti}$ & 1,799 & 21.5 & 915 & 372 & -50.0 & 865 & 58 & 51 \\
\hline $\mathrm{Ar}, \mathrm{Ti}$ & 1,716 & 15.5 & 1,030 & 455 & -39.0 & 730 & 300 & 50 \\
\hline
\end{tabular}




\section{Chapter 3}

\section{First Measurement of the $\operatorname{Ti}\left(e, e^{\prime}\right) X$ Cross Section at Jefferson Lab}

The work described in this chapter was published as:

Hongxia Dai, Matt Murphy, Vishvas Pandey, et al, (The Jefferson Lab Hall A Collaboration), "First measurement of the $\operatorname{Ti}\left(e, e^{\prime}\right) X$ Cross Section at Jefferson Lab," Phys. Rev. C, 98, 014617 (2018) [92].

Reproduced with permission from the American Physical Society. Copyright (2019) by the American Physical Society.

I contributed the main data analysis work in this paper under Professor Camillo Mariani's supervision.

\subsection{Introduction}

The interpretation of the data collected by experimental studies of neutrino oscillations demands a fully quantitative description of neutrino interactions with the atomic nuclei comprising the detector [40]. Current and future neutrino experiments, such as the short(SBN) [41] and the long-baseline (DUNE) [42] neutrino programs, will use detectors based on the liquid-argon time-projection chambers (LArTPCs) technology. In order to achieve 
the precision goals of these programs, the treatment of nuclear effects, which has been recognized as a major source of systematic uncertainty in ongoing experiments [43], has to be addressed. Realistic models of both neutrino- and antineutrino-argon interactions will be even more critical to future experiments, such as DUNE, aimed at pinning down charge-parity (CP) symmetry violation in the leptonic sector, because its determination with few percent precision requires accurate measurements of both neutrino and antineutrino oscillations. Failing to achieve this goal in a timely manner will deeply affect the sensitivity of DUNE to measure the CP violating phase $\left(\delta_{C P}\right)$, as discussed in [44].

Since the description of nuclear effects in a non isospin-symmetric nucleus, such as argon, must take into account the differences in the shell-model states occupied by protons and neutrons, and the information on neutrons cannot be directly extracted, one has to resort to studies of titanium nucleus. Owing to the fact that the neutron spectrum of ${ }_{18}^{40} \mathrm{Ar}$ is mirrored by the proton spectrum of $\mathrm{Ti}$, the $\mathrm{Ti}$ data will give access to the neutron spectral function of argon. Given the scarcity of electron-argon scattering experiments - the only available data on argon being the inclusive spectrum measured at Frascati National Laboratory using the electron-positron collider ADONE and a jet target [45] — we performed a dedicated experiment at Jefferson Lab (JLab), aimed at measuring inclusive and exclusive cross-sections on both argon and titanium targets, and extracting the proton [via $\left.\operatorname{Ar}\left(e, e^{\prime} p\right)\right]$ and neutron [via $\left.\left.\operatorname{Ti}\left(e, e^{\prime} p\right)\right)\right]$ spectral functions of the argon nucleus in the kinematical region in which shell-model dynamics is dominant [29].

Electron-scattering experiments have provided a wealth of information on the nuclear response to electromagnetic interactions over a broad kinematic regime. Static form factors and charge distributions have been extracted from elastic scattering data, while measurements of inelastic cross sections have allowed for systematic studies of the dynamic response functions, which shed light on the role played by different reaction mechanisms. Finally, with 
Chapter 3. First Measurement of the Ti $\left(e, e^{\prime}\right) X$ Cross Section at Jefferson 50

$\mathrm{LAB}$

the advent of continuous beam accelerators, a number of exclusive processes have been analyzed to unprecedented precision. The availability of the body of electron-nucleus scattering data has been essential to the development of theoretical models. Most notably, experimental studies of the $\left(e, e^{\prime} p\right)$ process - in which the scattered electron and the knocked-out proton are detected in coincidence - have provided detailed information on proton spectral functions [46, 47, 48,49], the knowledge of which is needed to obtain the nuclear cross sections within the factorization scheme underlying the impulse approximation (IA). The description based on the IA and the spectral function formalism [50] has been successful in describing inclusive electron-scattering data in a variety of kinematic regimes [51, 52, 53], and has recently been be extended to the analysis of neutrino scattering $[54,55,56,57]$. However, due to the scarcity of available inclusive cross sections for argon and titanium, theoretical models of nuclear effects in electroweak interactions [58, 59, 60, 61, 62, 63, 64] cannot be currently validated in the kinematical region relevant for neutrino experiments.

The experiment E12-14-012 planned to perform a combined analysis of Ar and Ti inclusive and exclusive reactions collected high statistics data in JLab Hall A during February-March 2017. Here, we report the first results of the experiment, including the $\operatorname{Ti}\left(e, e^{\prime}\right) X$ cross section at beam energy $E=2.222 \mathrm{GeV}$ and electron scattering angle $\theta=15.541$ deg and the corresponding electron-carbon cross section. Note that this is the first double differential $\operatorname{Ti}\left(e, e^{\prime}\right) X$ cross section measured at the kinematics relevant for neutrino experiments, the previous studies on titanium target include [65, 66, 67]. The measurement of the $\mathrm{C}\left(e, e^{\prime}\right) X$ cross section allowed a comparison with previous experiments, as well as a careful study of systematic uncertainties. In the $\left(e, e^{\prime}\right)$ process $e+A \rightarrow e^{\prime}+X$, an electron of four-momentum $k \equiv(E, \mathbf{k})$ scatters off a nuclear target $A$. The energy and emission angle of the scattered electron of four-momentum $k^{\prime} \equiv\left(E^{\prime}, \mathbf{k}^{\prime}\right)$ are measured, while the hadronic final state is left undetected. The squared four-momentum transfer in the process is $q^{2}=-Q^{2}$, with 
$q=k-k^{\prime} \equiv(\omega, \mathbf{q})$

\subsection{Experimental Setup}

The electron beam was provided by the Continuous Electron Beam Accelerator Facility (CE$\mathrm{BAF})$ at JLab, with currents in excess of $10 \mu \mathrm{A}$. The beam current was monitored using two Beam Current Monitors (BCMs), which are resonant radio-frequency cavities. The position of the beam was monitored by two similar cavity types, Beam Position Monitors (BPMs). Beam size was measured with harp scanners, which also allowed the cavity monitor calibration. Beam position determination is important for vertex reconstruction and momentum calculation of the scattered electron. The beam was rastered, with a $2 \mathrm{~mm} \times 2 \mathrm{~mm}$ raster system, in both vertical and horizontal directions, to reduce the beam current density and hence eliminate the possibility of melting the solid foil targets and minimize the local heating of the cryogenic hydrogen target. Both the carbon and titanium targets were foils of natural isotope composition, with a thickness of $0.167 \pm 0.001 \mathrm{~g} / \mathrm{cm}^{2}$ and $0.729 \pm 0.001 \mathrm{~g} / \mathrm{cm}^{2}$, respectively.

The scattered particles were momentum analyzed by two nearly identical spectrometersthe Left and a Right High-Resolution Spectrometers (HRSs) - equipped with detectors for tracking, timing and particle ID. The HRSs consist of 4 magnets (3 superconducting and 1 resistive) in a QQDQ configuration, where the $\mathrm{Q}$ indicates a quadrupole magnet and the $\mathrm{D}$ indicates a dipole magnet. This arrangement provided a large acceptance for both the angle and momentum, with a relative momentum resolution of $\sim 10^{-4}$, and pointing accuracy and angular resolution of $\sim 10^{-4} \mathrm{~m}$ and $\sim 10 \mathrm{mrad}$, respectively. The detector package, slightly updated with respect to the one in Ref. [15], consisting of vertical drift chambers (VDCs), threshold Čerenkov counters, scintillator detectors and a lead-glass calorimeters, provides 
Chapter 3. First Measurement of the Ti $\left(e, e^{\prime}\right) X$ Cross Section at Jefferson 52

LAB

data-acquisition triggering, tracking, and particle identification.

The scattered electrons were detected in the Left HRS positioned at $\theta=15.541 \mathrm{deg}$. The data acquisition was triggered, with an efficiency of $99.9 \%$, when an electron fired the two scintillator detectors planes (with a logical AND) simultaneously with a signal in the gas Čerenkov detector. The electrons were identified by a gas threshold Čerenkov detector, mounted between two scintillator detector planes, with $99.9 \%$ efficiency and negligible pion contamination. The track trajectories were reconstructed in the detector stack using the VDCs with efficiencies of $\sim 95 \%$ for $\mathrm{C}$ and $\sim 92 \%$ for $\mathrm{Ti}$, and then transported to the target utilizing a fitted reconstruction matrix obtained from a special optics calibration run. We required only one VDC track reconstructed in the final state for simplicity and purity purposes. The number of VDC reconstructed tracks in the case of the Ti target is slightly higher than for the $\mathrm{C}$ target, the difference in respective VDC efficiencies was as expected.

\subsection{Data Analysis}

The cross section is extracted first computing the yield defined in both data and simulation as:

$$
\text { Yield }^{i}=\left(N_{S}^{i} \times \mathrm{DAQ}_{\text {pre-scale }}\right) /\left(N_{e} \times L T \times \epsilon\right)
$$

Here, $i$ is the $i$ th bin in $E^{\prime}, N_{S}^{i}$ represents the number of scattered electrons, $N_{e}$ is the total number of electrons on the target, $L T$ is the live-time, $\epsilon$ is the total efficiency and $\mathrm{DAQ}_{\text {pre-scale }}$ is a factor that determines what fraction of the events gets recorded. The cross section in each bin $i$ is computed as the product of the Monte Carlo (MC) cross section [68] times the ratio of the data to simulation yields. The MC cross section is a fit to existing data, including preliminary Hall C [69] data and includes radiative corrections computed using the peaking approximation [22] and Coulomb corrections implemented with an effective 


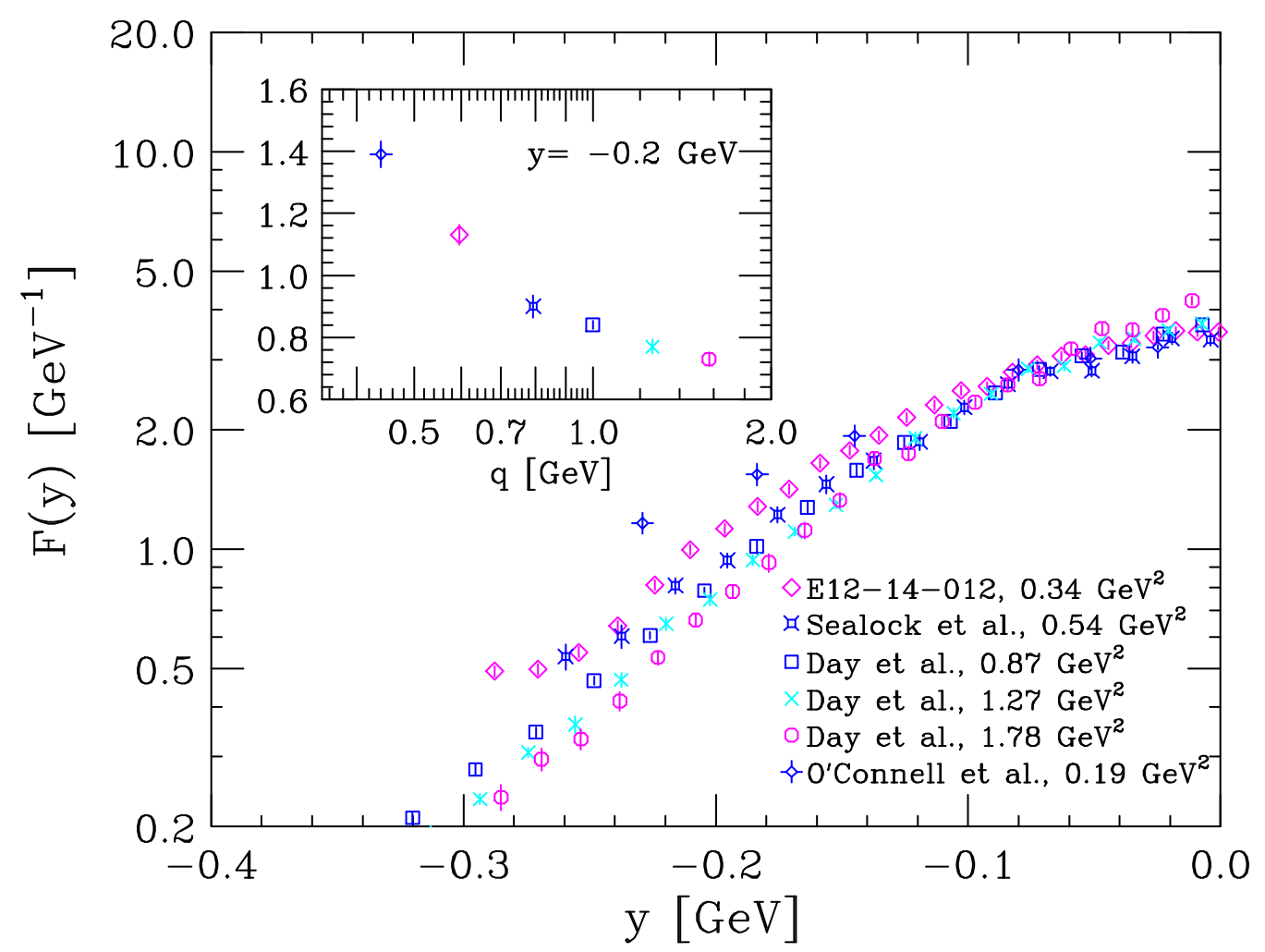

Figure 3.1: Comparison between the scaling function $F(y)$ obtained from the E12-14-012 data on C, represented by diamonds, and those obtained from the data of O'Connell et al. [74], Sealock et al. [75], and Day et al. [76]. The inset shows the momentum transfer dependence of $F(y)$ at fixed $y=-0.2 \mathrm{GeV}$. The data sets are labeled by the value of $Q^{2}$ corresponding to the top of the quasielastic peak.

momentum approximation [70].

\subsection{Experimental Results and Discussion}

Over nearly five decades, a number of measurements of the electron-carbon cross section have been performed at different electron-scattering facilities around the world. A compilation of the available inclusive data can be found in Ref. [71]. In order to put our results in perspective, in Fig. 3.1 we compare the $y$-scaling function [72], $F(y)$, obtained from the 
Chapter 3. First Measurement of the Ti $\left(e, e^{\prime}\right) X$ Cross Section at Jefferson 54

$\mathrm{LAB}$

cross section measured by the E12-14-012 experiment to those obtained from the data of Refs. [74, 75, 76], spanning a kinematical range corresponding to $0.20 \lesssim Q^{2} \lesssim 1.8 \mathrm{GeV}^{2}$. The occurrence of scaling in the variable, i.e. the observation that $F(y)$ becomes independent of the momentum transfer $|\mathbf{q}|$ in the limit of large $|\mathbf{q}|$, indicates that quasielastic scattering is the dominant reaction mechanism and final state interactions (FSI) between the knocked out nucleon and the residual nucleus are negligible. The scaling variable $y$, whose definition is given in Ref. [72], can be loosely identified with the component of the initial nucleon momentum parallel to the momentum transfer.

The main panel of Fig. 3.1 clearly shows that the data exhibit a remarkable scaling behavior at $y \approx 0$, corresponding to $\omega \approx Q^{2} / 2 M$, where $M$ is the nucleon mass, while sizable scaling violations, to be mainly ascribed to FSI, are observed at large negative values of $y$. The momentum transfer dependence of $F(y)$ at $y=-0.2 \mathrm{GeV}$, illustrated in the inset, demonstrates that in the kinematical setup of our experiment, corresponding to $|\mathbf{q}| \approx 600 \mathrm{MeV}$, the effects of FSI are still significant. Overall, Fig. 3.1 shows that our results are fully consistent with those of previous experiments.

Table 3.1: Contributions to the uncertainties associated with the measured $\mathrm{C}\left(e, e^{\prime}\right)$ cross sections. Numbers represents upper limit or range for the uncertainties that vary between kinematical regions.

\begin{tabular}{lc}
\hline \hline 1. Total statistical uncertainty & $1.2 \%$ \\
2. Total systematic uncertainty & $2.0-2.9 \%$ \\
a. Beam charge \& Beam Energy & $0.3 \%$ \\
b. Beam offset $x \& y$ & $0.1 \%-0.4 \%$ \\
c. Target thickness & $0.1 \%-0.4 \%$ \\
d. HRS offset $x \& y+$ Optics & $1.3 \%-2.0 \%$ \\
e. Acceptance cut $(\theta, \phi, d p / p)$ & $1.0 \%-1.4 \%$ \\
f. Calorimeter \& Cerenkov cuts & $0.01 \%-0.02 \%$ \\
g. Cross Section Model & $0.1 \%-0.2 \%$ \\
h. Radiative +Coulomb Corr. & $1.0-1.3 \%$ \\
\hline \hline
\end{tabular}




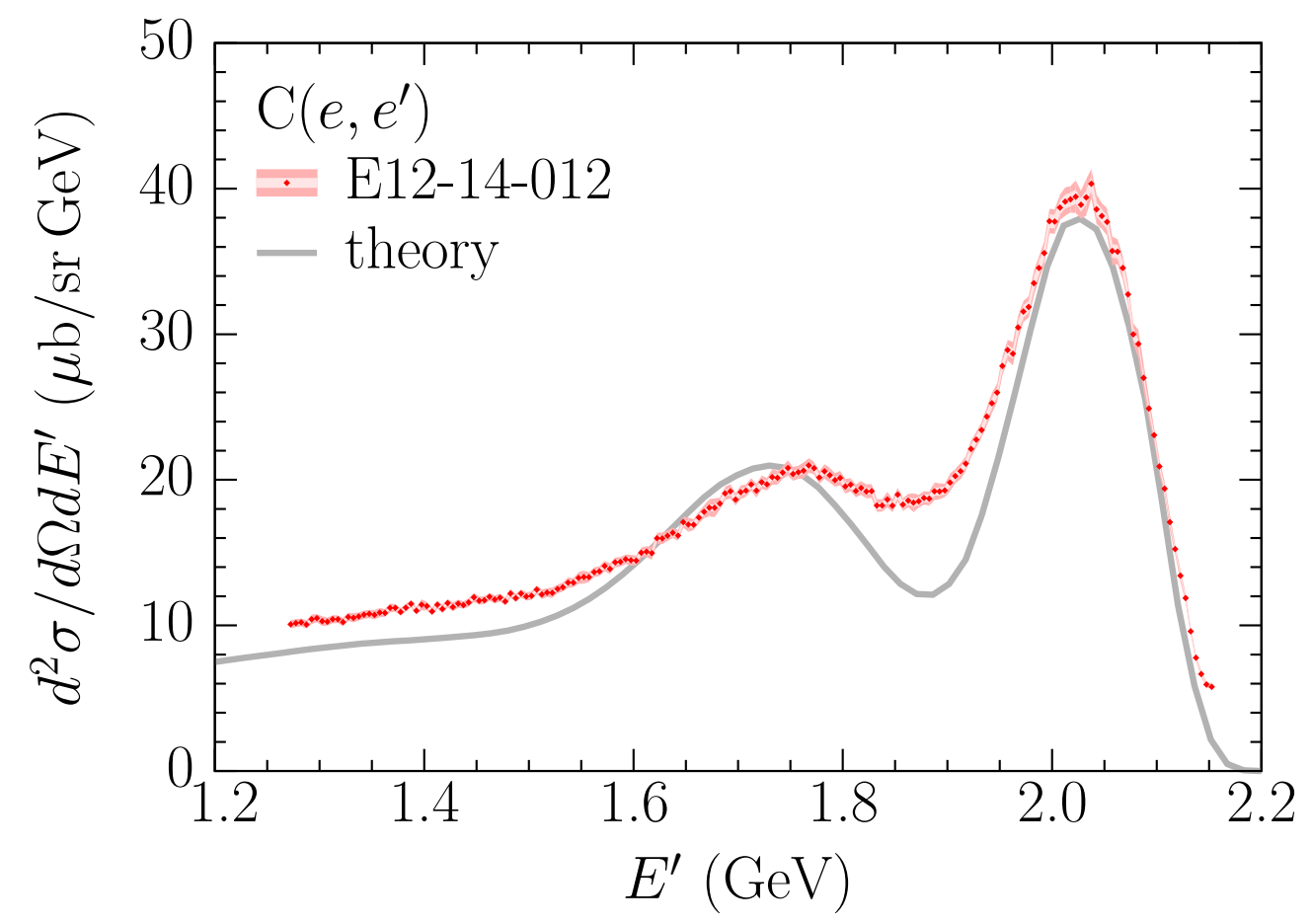

Figure 3.2: Double differential cross section for the $\mathrm{C}\left(e, e^{\prime}\right)$ process measured at beam energy of $2.222 \mathrm{GeV}$ and scattering angle of $15.541 \mathrm{deg}$. The inner and outer uncertainty bars correspond to statistical and total uncertainties, respectively. The solid line represents the theoretical results obtained within the formalism described in Refs. [50, 52, 53, 81].

Figure 3.2 shows the measured $\mathrm{C}\left(e, e^{\prime}\right)$ cross section as a function of the energy of the scattered electron, ranging from $\sim 1.2 \mathrm{GeV}$ to $\sim 2.2 \mathrm{GeV}$ with error bars up to $\sim 2.5 \%$, corresponding to the statistical $(1.2 \%)$ and systematic $(2.2 \%)$ uncertainties summed in quadrature. It can be seen that the kinematical coverage includes both the quasielastic and delta-production peaks, and extends to the region in which the contribution of deep-inelastic scattering becomes appreciable. The statistical uncertainty includes beam charge (0.03\%), detector and trigger efficiencies $(0.1 \%)$, DAQ live-time $(0.02 \%)$, VDC, and VDC track reconstruction efficiencies $(0.1 \%)$ and uncertainties due to the charge-symmetric background prediction [77] $(0.01 \%)$. A detailed list of the systematic uncertainties is given in Table 3.1. All uncertainties are considered as fully uncorrelated. This new high precision $\mathrm{C}\left(e, e^{\prime}\right)$ data not only allowed us to carefully test our analysis framework and study systematics but also pro- 
Chapter 3. First Measurement of the Ti $\left(e, e^{\prime}\right) X$ Cross Section at Jefferson 56

LAB

vides a vital information for the neutrino experiments that use carbon targets such as the long-baseline neutrino experiment T2K [78], NOvA [79] and neutrino interaction experiment MINERvA [80].

The solid line of Fig. 3.2 represents theoretical results obtained within the scheme described in Refs. $[50,52,53,81]$, based on the factorization ansatz dictated by the IA and the spectral function formalism. Note that this approach does not involve any adjustable parameters, and allows for a consistent inclusion of single-nucleon interactions - both elastic and inelasticand meson-exchange current (MEC) contributions. The effects of FSI on the quasielastic cross section has been taken into account following the procedure developed in Ref. [81]. A detailed account of the calculation of the electron-carbon cross section will be provided in a forthcoming paper [82].

Figure 3.3 presents the inclusive electron-titanium cross section, measured at the same kinematics as for carbon and with an error up to $~ 2.75 \%$, sum in quadrature of statistical (1.65\%) and systematic (2.2\%) uncertainties. In the absence of any previous electron-scattering studies carried out using a titanium target, we determined the $\operatorname{Ti}\left(e, e^{\prime}\right)$ cross sections using:

$$
\left(\frac{d^{2} \sigma^{\text {Born }}}{d \Omega d E^{\prime}}\right)_{\mathrm{Ti}}^{i}=\left(\frac{d^{2} \sigma^{\text {Born }}}{d \Omega d E^{\prime}}\right)_{\mathrm{C}}^{i} \times \frac{\text { Yield }_{\mathrm{Ti}}^{i}}{\text { Yield }_{\mathrm{C}}^{i}}
$$

where Yield $_{\mathrm{C} / \mathrm{Ti}}^{i}$ denotes the luminosity normalized yield respectively for $\mathrm{C}$ and Ti. By normalizing the yield ratio to published radiatively unfolded carbon cross sections $d \sigma_{C}^{\text {Born }}$, we are implicitly unfolding bremsstrahlung from the quoted Ti cross sections. In this approach, most of the systematic uncertainties are fully correlated between $\mathrm{C}$ and $\mathrm{Ti}$, due to the fact that the data was collected in the same kinematical setup and analyzed using the same cuts of the carbon data. Uncertainties due to radiative corrections, target thickness and density were evaluated independently for $\mathrm{Ti}$, and added in quadrature to the uncertainties from 


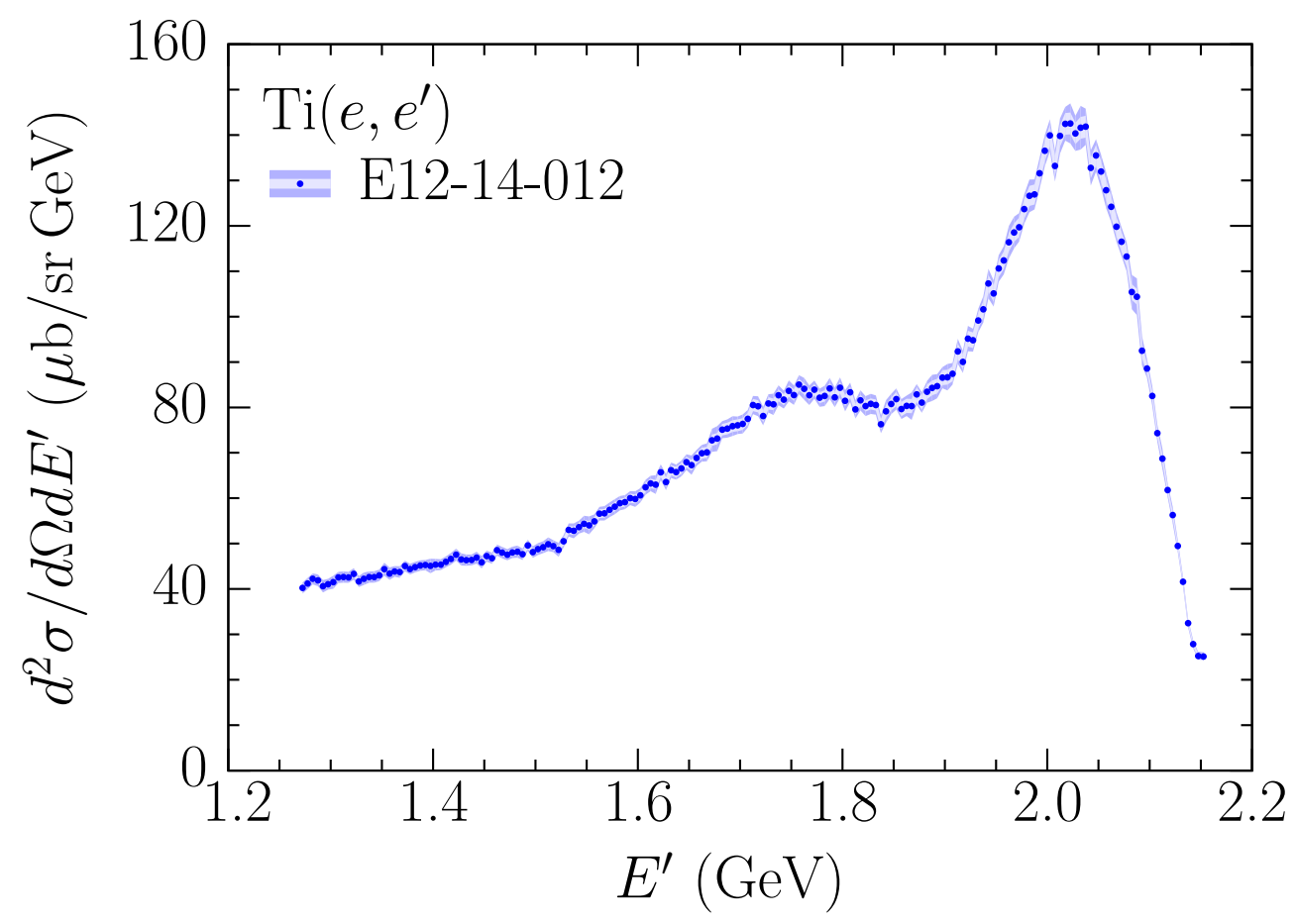

Figure 3.3: Double differential cross section for the $\operatorname{Ti}\left(e, e^{\prime}\right)$ process measured at beam energy of $2.222 \mathrm{GeV}$ and fixed scattering angle of $15.541 \mathrm{deg}$. The inner and outer uncertainty bars correspond to statistical and total uncertainties, respectively. The maximum uncertainties in the full kinematical range are provided.

C. Note that this is the first electron-titanium scattering data collected at the kinematics relevant for neutrino experiments. Therefore, the model of Refs. [50, 52, 53, 81], requiring as an input the target spectral function, could not be used to obtain theoretical results comparable to the data of Fig. 3.3.

Figure 3.4 shows the ratio

$$
\left(d^{2} \sigma / d \Omega d E^{\prime}\right) /\left[Z \sigma_{e p}+(A-Z) \sigma_{e n}\right]
$$

for carbon and titanium,. Here $\sigma_{e p}$ and $\sigma_{e n}$ denote the elastic electron-proton and electronneutron cross sections stripped of the energy-conserving delta function. The difference between the results obtained using the measured carbon and titanium cross sections reflect 
Chapter 3. First Measurement of the Ti $\left(e, e^{\prime}\right) X$ Cross Section at Jefferson 58

$\mathrm{LAB}$

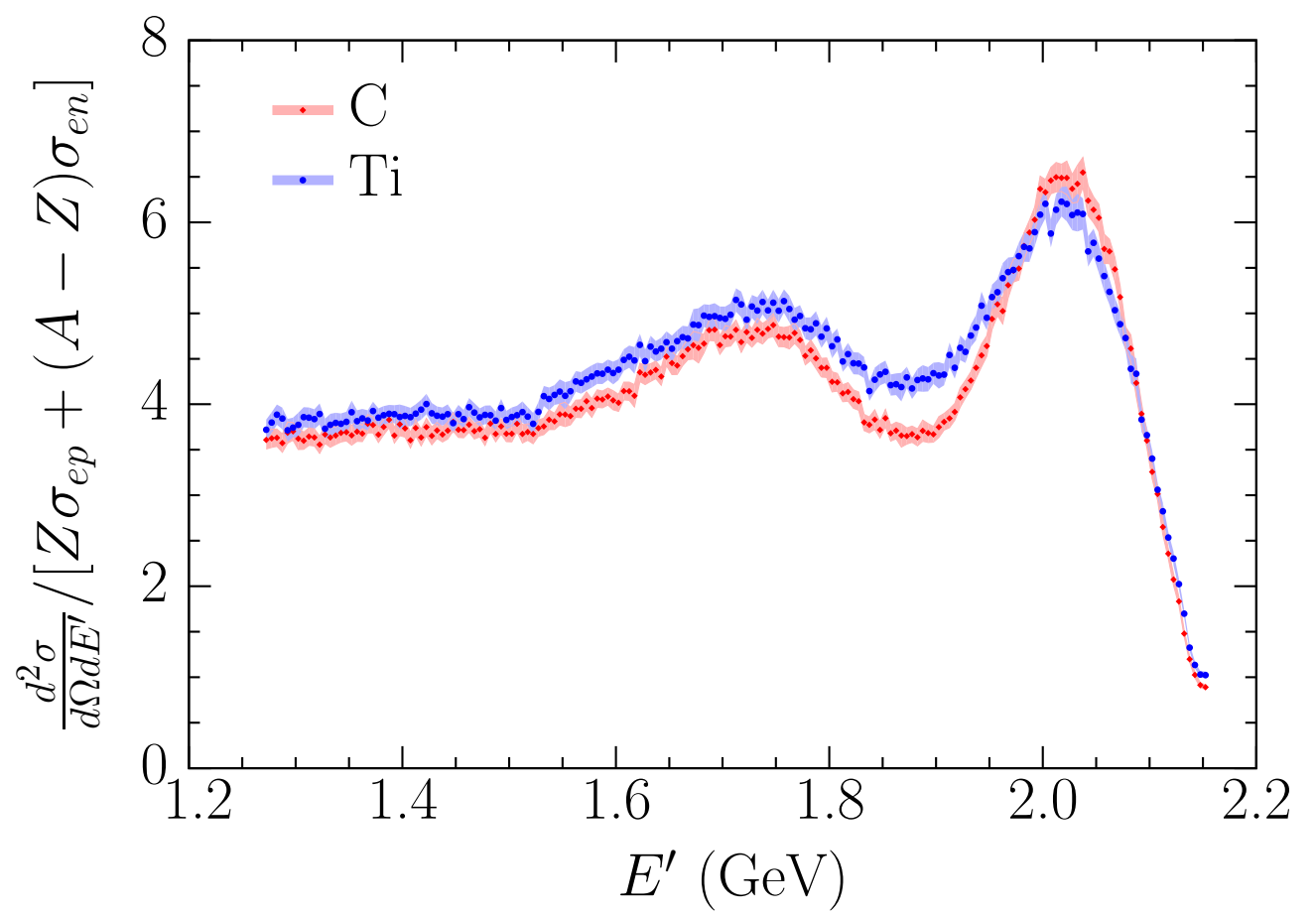

Figure 3.4: Ratios defined by Eq.(3.3), computed using the measured carbon and titanium cross sections.

different nuclear effects, that can be conveniently parametrized in terms of a nuclear Fermi momentum exploiting the concept of scaling of second kind, or superscaling [83]. The superscaling analysis of our data, illustrated in Fig. 3.5, suggests that the Fermi momentum in titanium is $\sim 240 \mathrm{MeV}$, to be compared to $220 \mathrm{MeV}$ in carbon [84].

\subsection{Summary and Conclusions}

In this Letter, we have reported the first results of JLab experiment E12-14-012, consisting of the $\mathrm{Ti}\left(e, e^{\prime}\right)$ and $\mathrm{C}\left(e, e^{\prime}\right)$ cross sections at beam energy $E=2.222 \mathrm{GeV}$ and scattering angle $\theta=15.541 \mathrm{deg}$. The quality of the CEBAF electron beam and the excellent performances of the high resolution spectrometer and detector packages available in Hall A allowed for a quick and smooth data taking, and an accurate determination of the cross sections over the 


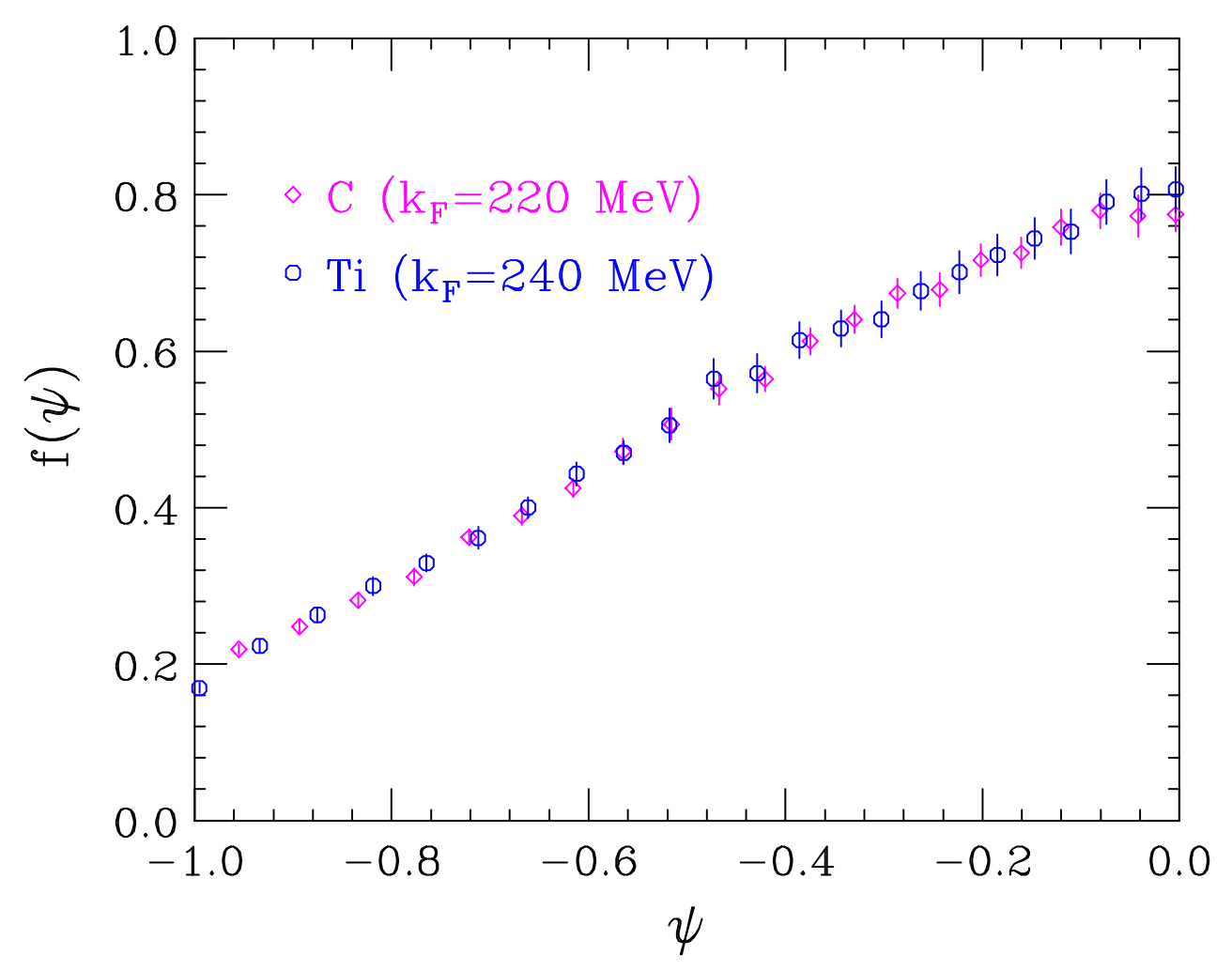

Figure 3.5: Comparison between the scaling function of $2^{\text {nd }}$ kind, $f(\psi)$, obtained from the E12-14-012 data on $\mathrm{C}$ and $\mathrm{Ti}$, represented by diamonds and circles, respsctively. The Fermi momentum of carbon has been fixed to the value obtained by Moniz et al. [84]. The data analysis for Ti sets the Ti Fermi momentum at $\sim 240 \mathrm{MeV}$.

broad range of energy transfer in which quasielastic scattering -induced by both one- and two-nucleon currents - and resonance production are the main contributions to the inclusive cross sections.

Our titanium measurement, providing first electron-titanium scattering data at the kinematics relevant for neutrino experiments, will be of great value for the development of realistic models of the electroweak response of neutron-rich nuclei, which will be indispensable for the analysis of the next generation of neutrino oscillation studies employing argon detectors such as DUNE. Our carbon measurements provide a high precision data that can be utilized 
Chapter 3. First Measurement of the Ti $\left(e, e^{\prime}\right) X$ Cross Section at Jefferson 60

$\mathrm{LAB}$

in the neutrino experiments that use carbon targets such as T2K, NOvA and MINERvA. Comparison between the results of theoretical calculations and carbon data confirms that the approach based on the spectral function formalism, supplemented by the inclusion of MEC and FSI contributions, provides a consistent framework, capable of providing a parameter free description of electron-nucleus scattering in the kinematical regime in which the IA is expected to be applicable. 


\section{Chapter 4}

\section{First Measurement of the $\operatorname{Ar}\left(e, e^{\prime}\right) X$ Cross Section at Jefferson Lab}

The work described in this chapter was published as:

Hongxia Dai, Matt Murphy, Vishvas Pandey, et al, (The Jefferson Lab Hall A Collaboration), "First measurement of the $\operatorname{Ar}\left(e, e^{\prime}\right) X$ Cross Section at Jefferson Lab," Phys. Rev. C, 99, 054608 (2019) [101].

Reproduced with permission from the American Physical Society. Copyright (2019) by the American Physical Society.

I contributed the data analysis work in this paper under Professor Camillo Mariani's supervision.

\subsection{Introduction}

Precise determination of charge-parity $(\mathrm{CP})$ symmetry violation in the lepton sectornecessary to shed light on the matter-antimatter asymmetry in the Universe - is among the highest priorities of particle physics. Over the next two decades, this issue will be a primary science goal of the Deep Underground Neutrino Experiment (DUNE) [85], together with a search for proton decay, measurement of the electron-neutrino flux from a core-collapse 
Chapter 4. First Measurement of the Ar $\left(e, e^{\prime}\right) X$ Cross Section at Jefferson 62

supernova - should one occur in our galaxy during the lifetime of DUNE - and search for physics beyond the standard model.

In the next few years, the Short-Baseline Neutrino (SBN) program [41] at Fermilab will provide definitive answer to the question of existence of sterile neutrinos, which could be the source of electron-like events recently reported with statistical significance $4.8 \sigma$ by the MiniBooNE collaboration [86].

Both DUNE and SBN program (will) employ liquid-argon time-projection chambers as their detectors, the advantages of which are low threshold momenta for particle detection and high spatial resolution, allowing (among others) for precise neutrino-energy reconstruction and distinguishing photons from electrons. As a consequence, the success of both programs in studying neutrino oscillations with unprecedented precision will greatly rely on the precision with which we understand the complexity of nuclear effects in argon and the precision with which we are able to estimate its response to electroweak probes.

It is important to realize that, although the near detector facilities of DUNE will play a fundamental role in the reduction of systematic uncertainties, yet alone they will not be sufficient to determine the cross sections with the precision necessary to achieve the objectives of DUNE [42]. At beam energies in the few-GeV region, the observed event kinematics cannot be readily translated to the true value of neutrino energy, owing to detector effects, and the procedure of energy reconstruction heavily relies on the nuclear model used in Monte Carlo (MC) simulations [87]. Even for functionally identical near and far detectors, the spectrum reconstructed in the near detector is very different from the one in the far detector. This is a consequence of not only neutrino oscillations, but also of differences in particle containment and angular acceptance, and of the strong angular dependence of the flux, which makes important the difference between the solid angle probed by near and far detectors, even in absence of the oscillations. As CP-violation sensitivity of DUNE critically 
depends on systematic uncertainties, even their modest reduction has a meaningful impact on the running time necessary to achieve the physics objectives.

In the ongoing oscillation experiments $[88,89]$, the uncertainties related to nuclear effects in neutrino-nucleus interactions have become one of the major sources of systematics [40, 90], despite extensive use of near-detector data to constrain the nuclear models employed in MC simulations. As different probe's energies and reaction mechanisms are intertwined in neutrino-scattering data, it is difficult to identify, diagnose, and remedy potential shortcomings of nuclear models. On the other hand, electron-scattering measurements with targets and kinematics of interest to neutrino experiments give an excellent opportunity to validate and improve the description of nuclear effects [91]. Considering that there is a large body of electron-scattering data available for carbon (and limited availability of data for oxygen) the situation for argon is woefully inadequate, with only one dataset currently available: the inclusive electron-scattering spectrum measured at Frascati National Laboratory (LNF) using the electron-positron collider ADONE and a jet target at incident electron energy $E=700 \mathrm{MeV}$ and scattering angle $\theta=32 \mathrm{deg}$ [45]. Argon can be expected to be more challenging to describe than oxygen and carbon, as a significantly heavier nucleus that is additionally isospin asymmetric. This asymmetry is of fundamental importance for the CP-violation measurement in DUNE, to be based on analysis of the difference between the neutrino and antineutrino event distributions. Availability of a new precise dataset for electron scattering off argon is therefore vital, in order to provide a testbed and stimulate further development of theoretical models of nuclear response to electroweak interactions $[52,53,57,58,59,60,61,62,63,64]$ in the kinematic region of interest to neutrino experiments.

To address this issue, we performed a dedicated experiment at Jefferson Lab (JLab) to study electron scattering from argon and titanium nuclei [29]. The experiment, E12-14-012, 
Chapter 4. First Measurement of the Ar $\left(e, e^{\prime}\right) X$ Cross Section at Jefferson 64

$\mathrm{LAB}$

collected high statistics data in JLab Hall A during February-March 2017. We have recently reported $\operatorname{Ti}\left(e, e^{\prime}\right) X$ and $\mathrm{C}\left(e, e^{\prime}\right) X$ cross section results [92]. Here, we present the first argon results of the experiment, $\operatorname{Ar}\left(e, e^{\prime}\right) X$ cross section at beam energy $E=2.222 \mathrm{GeV}$ and electron scattering angle $\theta=15.541 \mathrm{deg}$, and its comparison with our previously reported cross sections for the titanium and carbon nuclei in the same kinematics [92].

In the analyzed $\left(e, e^{\prime}\right)$ process, $e+A \rightarrow e^{\prime}+X$, an electron of four-momentum $k \equiv(E, \mathbf{k})$ scatters off a nuclear target $A$. The energy and scattering angle of the outgoing electron of four-momentum $k^{\prime} \equiv\left(E^{\prime}, \mathbf{k}^{\prime}\right)$ are measured while the hadronic final state remains undetected. The squared four-momentum transfer in the process is $q^{2}=-Q^{2}$, with $q=k-k^{\prime} \equiv(\omega, \mathbf{q})$.

\subsection{Experimental Setup}

A continuous-wave electron beam of energy $E=2.222 \mathrm{GeV}$ was supplied by the Continuous Electron Beam Accelerator Facility (CEBAF) at JLab. The current and position of the beam, the latter being critical for vertex reconstruction and momentum calculation of scattered electrons, were monitored by resonant radio-frequency cavities (Beam Current Monitors or $\mathrm{BCMs}$ ) and cavities with four antennae (Beam Position Monitors or BPMs), respectively. Harp scanners, which moved a thin wire through the beam, were used to measure its size. To eliminate the possibility of overheating the target by the deposited beam energy, the beam was rastered with a $2 \mathrm{~mm} \times 2 \mathrm{~mm}$ raster system, to increase the effective spot size and reduce the energy density.

The gaseous argon target, with a thickness of $1.455 \pm 0.005 \mathrm{~g} / \mathrm{cm}^{2}$, was contained in a $25 \mathrm{~cm}$ long cell with thin aluminum entry and exit windows of respectively $0.25 \mathrm{~mm}$ and $0.28 \mathrm{~mm}$ thickness. In order to account for the background contribution from electrons scattered from the wall of the argon target cell measurements were also performed on a dummy tar- 
get, aluminum foils mounted on separate frames located at positions corresponding to the entry and exit windows of the cell. The thickness of the entry and exit aluminum foils was $0.8886 \pm 0.002 \mathrm{~g} / \mathrm{cm}^{2}$ and $0.8893 \pm 0.002 \mathrm{~g} / \mathrm{cm}^{2}$, respectively and matched the radiation length of the argon target.

The scattered electrons were detected in the Left High-Resolution Spectrometer (LHRS) positioned at $\theta=15.541 \mathrm{deg}$. The LHRS was equipped with superconductive magnets and a detector package for tracking, timing and particle identification [15, 93]. The scattered electrons first passed through three superconducting quadrupole magnets (Q) and one dipole magnet (D) arranged in QQDQ configuration. This arrangement provided a large acceptance in both angle and momentum, and good resolution in momentum $\left(\sim 10^{-4}\right)$, position $\left(\sim 10^{-3} \mathrm{~m}\right)$ and in angle $(\sim 1.0 \mathrm{mrad})$. The electrons then entered the detector package consisting of vertical drift chambers (VDCs), threshold Čerenkov counter, scintillator detectors and a lead-glass calorimeter. The data-acquisition (DAQ) electronics was triggered when an electron passes through two scintillator detectors planes (with a logical AND) and simultaneously produces a signal in the gas $\mathrm{CO}_{2}$ Cerenkov counter, mounted between the two scintillator planes. Electron/pion separation is achieved with the combined amplitude response of the gas Čerenkov and Pb-Glass shower counters. The tracking information (position and direction) was reconstructed in the VDCs utilizing a reconstruction matrix obtained from special optics-calibration runs.

\subsection{Data Analysis}

The electron yield $(Y)$ for $i$ th bin in scattered electron energy $\left(E^{\prime}\right)$ is obtained as

$$
Y^{i}=\left(N_{S}^{i} \times D A Q_{\text {pre-scale }}\right) /\left(N_{e} \times L T \times \epsilon\right) .
$$


Chapter 4. First Measurement of the Ar $\left(e, e^{\prime}\right) X$ Cross Section at Jefferson 66

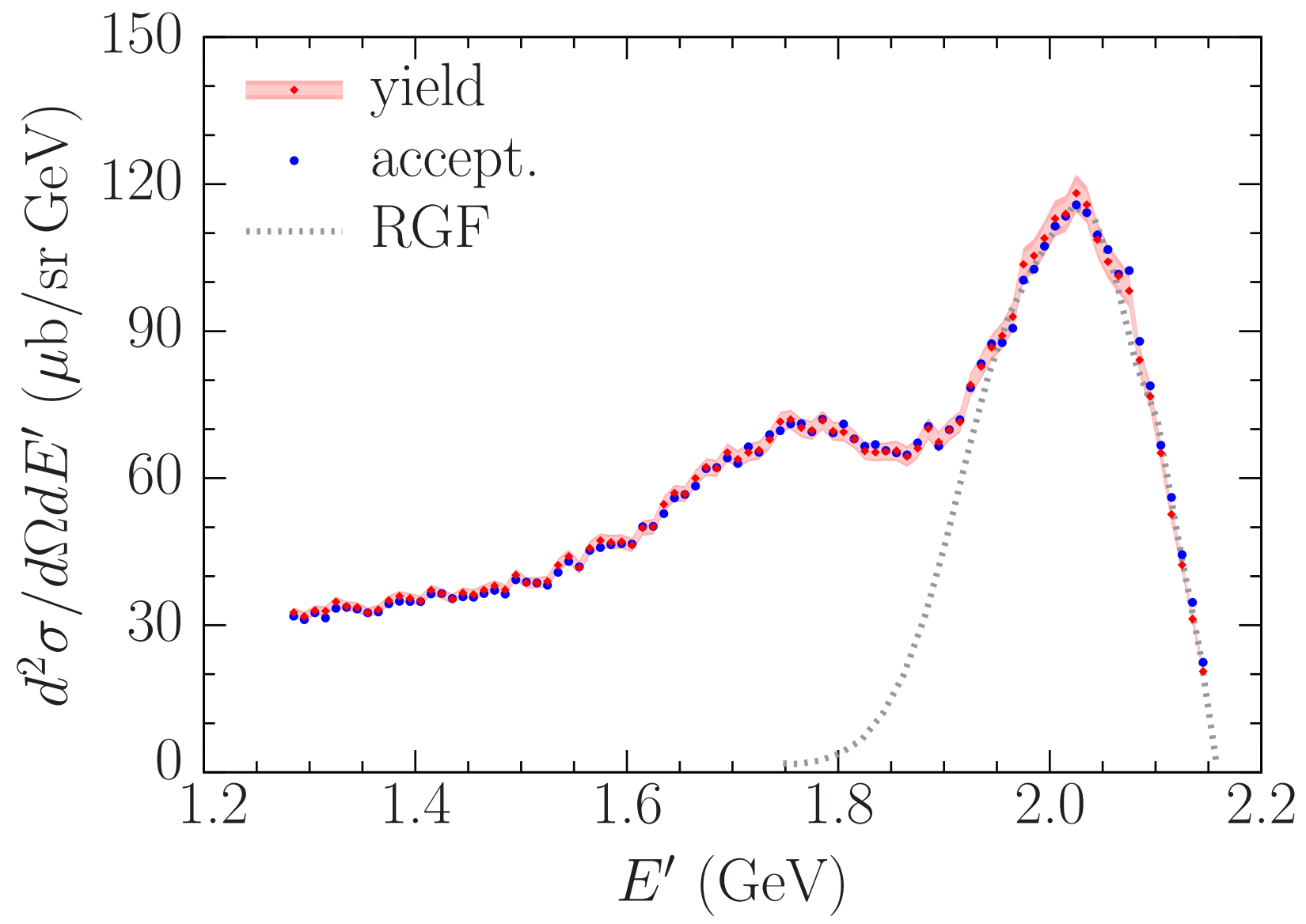

Figure 4.1: Double differential cross section for the $\operatorname{Ar}\left(e, e^{\prime}\right)$ process, extracted with two different methods, at beam energy of $2.222 \mathrm{GeV}$ and scattering angle of $15.541 \mathrm{deg}$. The inner and outer bars correspond to the statistical and total uncertainty, respectively. The dotted curve represents the quasielastic calculations obtained within the relativistic Green's function (RGF) formalism described in Ref. [94].

Here, $N_{S}^{i}$ is the number of scattered electrons recorded, $N_{e}$ is the total number of electrons incident on the target, $L T$ is the live-time fraction, $\epsilon$ is the total detection efficiency. The hardware trigger is configured to accept only every $n=D A Q_{\text {pre-scale }}$ raw triggers. The $\sim 10 \mu$ A beam rastered over $2 \times 2 \mathrm{~mm}^{2}$ deposits enough energy into the target that its density change must be taken into consideration when extracting the cross section. This is done through a target-boiling effect study in which the beam current is ramped in steps from zero current to $\sim 20 \mu \mathrm{A}$ and the scattering yield determined [37]. From this a correction to the 
zero current density can be made and applied to all the runs. The yield is also corrected for the background $(\sim 0.2 \%)$ remaining after the dummy cell is subtracted. Once the yield is determined, the cross section can be extracted either by the acceptance-correction method or by the yield-ratio method.

In the acceptance-correction method, for each bin in $\Delta E \Delta \Omega$, the cross section is obtained as

$$
d^{2} \sigma / d \Omega d E^{\prime}=Y\left(E^{\prime}, \theta\right) /\left[(\Delta E \Delta \Omega) A\left(E^{\prime}, \theta\right) L\right] .
$$

Where, $Y\left(E^{\prime}, \theta\right)$ and $A\left(E^{\prime}, \theta\right)$ are yield and acceptance for a given bin, respectively, and $L$ is the integrated luminosity obtained using a $\mathrm{MC}$ and validated with the solid $\mathrm{Al}$ target (dummy cell) and $\mathrm{C}$ foils (the optics target). In the yield-ratio method, the cross section for each bin is computed as the product of the MC cross section [68] times the ratio of the data to simulation yields

$$
d^{2} \sigma / d \Omega d E^{\prime}=\left(d^{2} \sigma / d \Omega d E^{\prime}\right)_{\mathrm{MC}} \times\left[Y\left(E^{\prime}, \theta\right) / Y_{\mathrm{MC}}\left(E^{\prime}, \theta\right)\right] .
$$

The MC cross section is a fit to the existing data including preliminary Hall $\mathrm{C}$ data [69]. The MC includes the radiative corrections computed using the peaking approximation [22] and Coulomb corrections implemented with an effective momentum approximation [70], further accounted for the change in radiation length of the target due to the target-boiling effect.

\subsection{Experimental Results and Scaling Analysis}

Figure 4.1 shows the measured $\operatorname{Ar}\left(e, e^{\prime}\right)$ double differential cross section as a function of the energy of the scattered electron, $E^{\prime}$, extracted with the yield-ratio and the acceptancecorrection method. Both methods yield the cross-section results in very good agreement, 
Chapter 4. First Measurement of the Ar $\left(e, e^{\prime}\right) X$ Cross Section at Jefferson 68

$\mathrm{LAB}$

with marginal differences observed only in the region of $E^{\prime}$ above the quasielastic peak (i.e. $\omega$ below the peak), where the event statistics are limited and the systematic uncertainties of the acceptance method are larger. The primary difference between the two methods is the fact that the yield-ratio method relies more on the predictions of the cross section model in the $\mathrm{MC}$ but the agreement of the two methods strengthens our confidence in both procedures. The measured cross section covers a broad range of scattered electron energy ranging from $\sim 1.3 \mathrm{GeV}$ to $\sim 2.2 \mathrm{GeV}$. The kinematical coverage includes both the quasielastic and delta-production peaks, and further extends to the deep-inelastic scattering region. The total uncertainties remain below $\sim 4.0 \%$ corresponding to the statistical $(1.7 \%-2.9 \%)$ and the systematic (1.8\%-3.0\%) uncertainties summed in quadrature. A detailed list of the uncertainties is given in Table 4.1.

Table 4.1: Uncertainties associated with the presented $\operatorname{Ar}\left(e, e^{\prime}\right)$ cross section. Numbers represent upper limits or the range for the uncertainties that vary between different kinematical regions.

\begin{tabular}{lc}
\hline \hline 1. Total statistical uncertainty & $1.7 \%-2.9 \%$ \\
2. Total systematic uncertainty & $1.8 \%-3.0 \%$ \\
a. Beam charge \& beam energy & $0.3 \%$ \\
b. Beam offset $x \& y$ & $0.4 \%-1.0 \%$ \\
c. Target thickness and boiling effect & $0.7 \%$ \\
d. HRS offset $x \& y+$ optics & $0.6 \%-1.2 \%$ \\
e. Acceptance cut $(\theta, \phi, d p / p)$ & $0.6 \%-2.4 \%$ \\
f. Calorimeter \& Cerenkov cuts & $0.01 \%-0.03 \%$ \\
g. Cross section model & $1.3 \%$ \\
h. Radiative \& Coulomb corrections & $1.0 \%$ \\
\hline \hline
\end{tabular}

The dotted curve of Fig. 4.1 represents the theoretical results obtained from the relativistic Green's function (RGF) approach described in Ref. [94]. In the RGF formalism, following assumptions based on the impulse approximation, the components of the nuclear response 


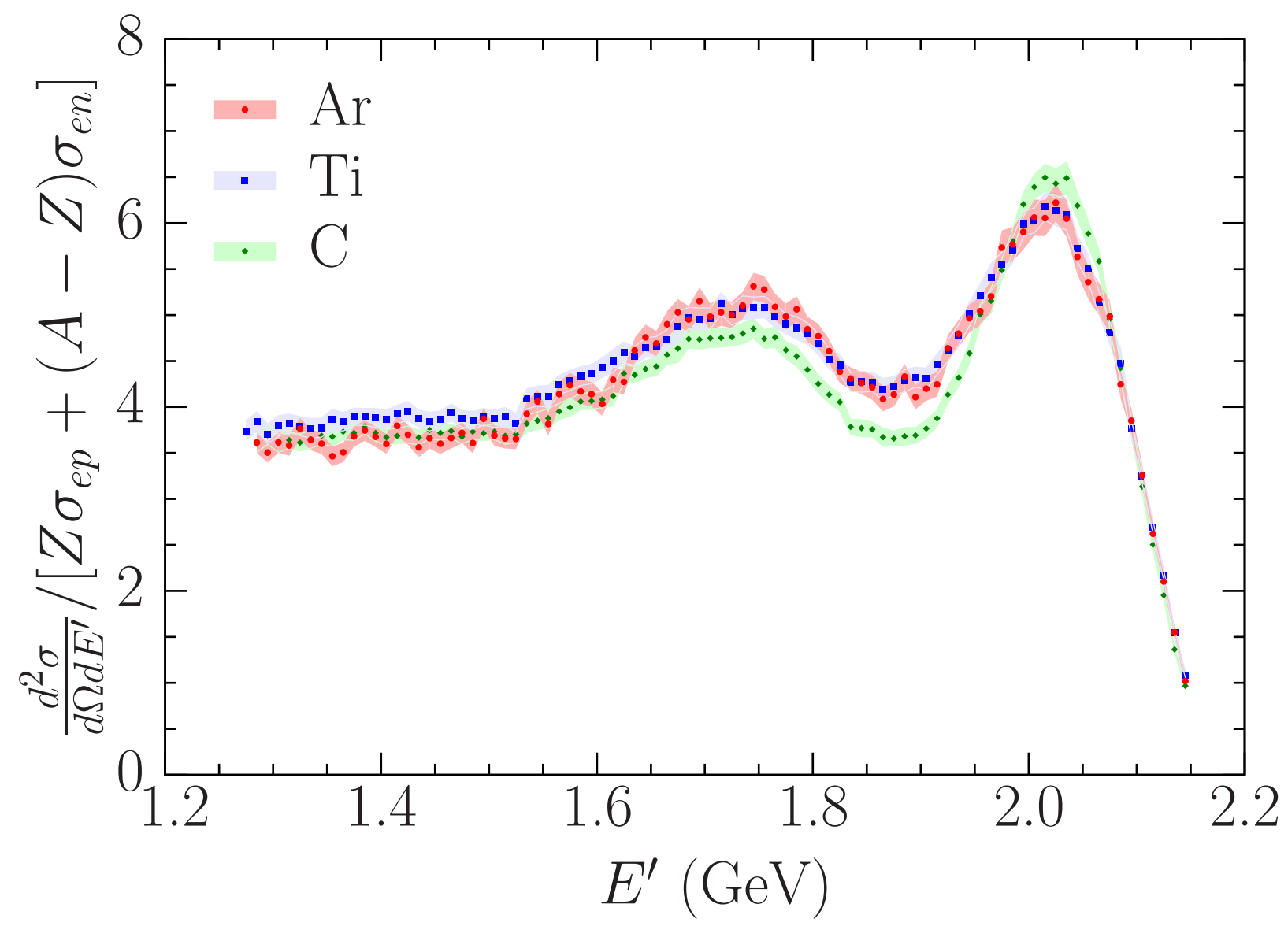

Figure 4.2: Comparison of $\operatorname{Ar}\left(e, e^{\prime}\right)$ cross section of Fig. 4.1, and $\operatorname{Ti}\left(e, e^{\prime}\right)$ and $\mathrm{C}\left(e, e^{\prime}\right)$ cross sections of Ref. [92], all in the same kinematics, presented in terms of the ratio defined by Eq.(4.4).

are written in terms of the single-particle optical-model Green's function. Final-state interactions are accounted for, consistently with the approach used in the exclusive $\left(e, e^{\prime} p\right)$ reaction, by the same complex optical potential but the formalism translates the flux lost towards inelastic channels, represented by the imaginary part of the optical potential, into the strength observed in inclusive reactions. It is apparent that this procedure leads to a remarkably good description of both shape and normalization of the data in the the quasielastic region. However, it does not include two-body currents and delta-excitation mechanisms which are clearly visible in the region of lower $E^{\prime}$ values (i.e. larger energy transfers). 
Chapter 4. First Measurement of the Ar $\left(e, e^{\prime}\right) X$ Cross Section at Jefferson 70

$\mathrm{LAB}$

In Fig. 4.2, we compare the argon data to the titanium and carbon data of Ref [92], taken in the same kinematical setup, corresponding to incident electron energy $2.222 \mathrm{GeV}$ and scattering angle of $15.541 \mathrm{deg}$. The comparison is performed in terms of the ratio defined as

$$
\left(d^{2} \sigma / d \Omega d E^{\prime}\right) /\left[Z \sigma_{e p}+(A-Z) \sigma_{e n}\right]
$$

where $A$ and $Z$ are the nuclear mass number and charge, respectively, while $\sigma_{e p}$ and $\sigma_{e n}$ denote the elastic electron-proton and electron-neutron cross sections stripped of the energyconserving delta function [95]. The results of Fig. 4.2, showing that the ratios of Eq.(4.4) corresponding to argon and titanium are nearly identical to one another, appear to support the strategy underlying our experiment, aimed at exploiting titanium data to extract complementary information on nuclear effects in argon. On the other hand, the differences between the results for argon and carbon indicate significant differences in the ground-state properties of these nuclei, which are relevant in the context of MC simulations for DUNE.

To further elucidate the differences between the argon, titanium, and carbon cross sections, in Fig. 4.3, we show the corresponding scaling functions of the second kind, $f(\psi)$, displayed as a function of the dimensionless scaling variable $\psi$. The definitions of both $f(\psi)$ and $\psi$ involve a momentum scale, which can be loosely interpreted as the nuclear Fermi momentum, $k_{F}$ [83], providing a simple parametrization of nuclear effects. The results of Fig. 4.3 show that setting the carbon Fermi momentum to $220 \mathrm{MeV}$ - the value resulting from the analysis of Moniz et al. [84] - scaling of titanium and argon data is observed for $k_{F}=240$ and $245 \mathrm{MeV}$, respectively. Hence, the scaling analysis confirms the picture emerging from Fig. 4.2. For comparison, we also show the scaling function $f(\psi)$ obtained using the $\operatorname{Ar}\left(e, e^{\prime}\right)$ cross section at $700 \mathrm{MeV}$ and $32 \mathrm{deg}$, measured at the LNF electron-positron storage ring ADONE using a jet target [45]. It turns out that scaling of the LNF data is only observed at $\psi \approx 0$ and prefers a much larger value of the Fermi momentum, $k_{F}=375 \mathrm{MeV}$, than the one resulting from the 


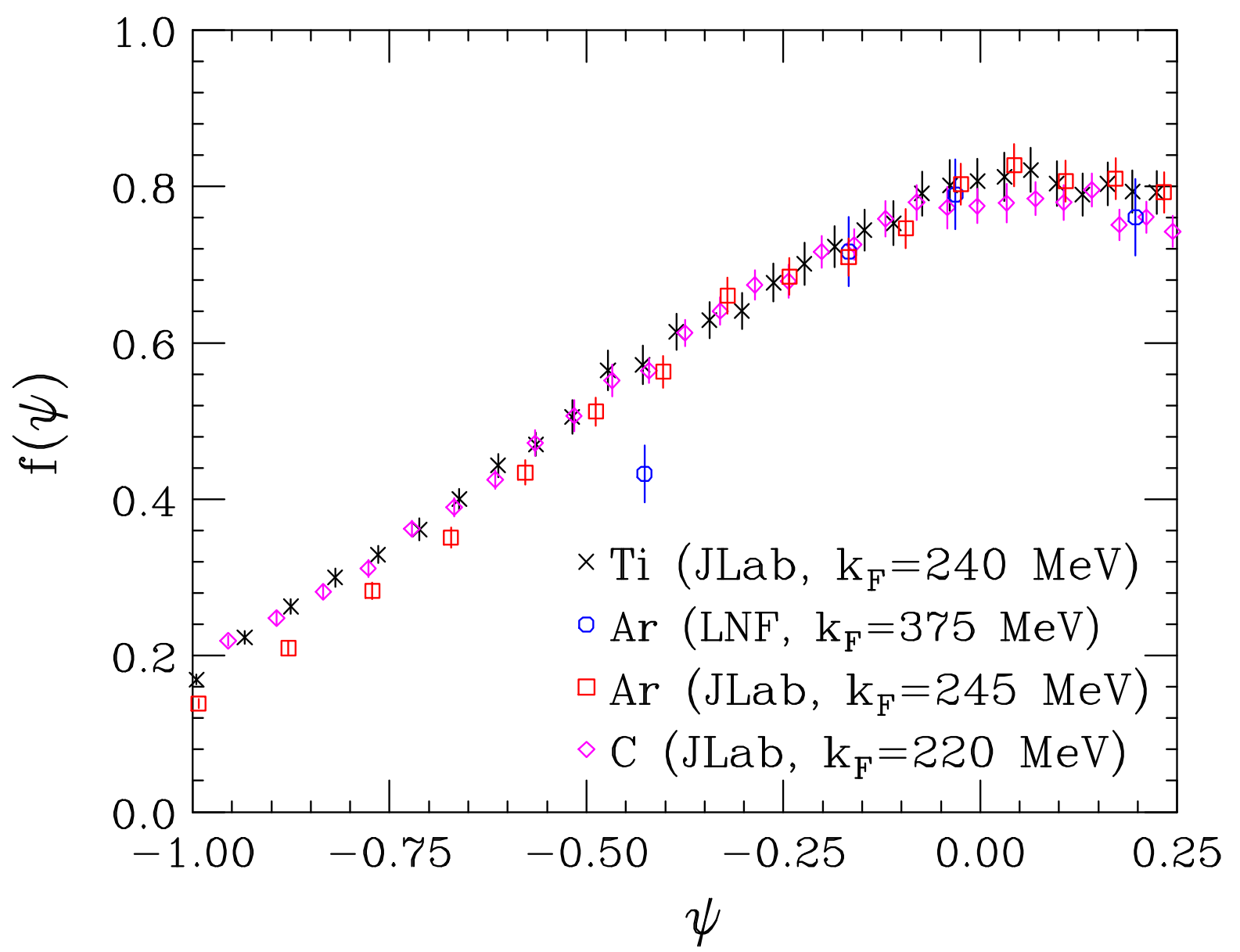

Figure 4.3: Comparison between the scaling function of the second kind, $f(\psi)$, obtained from E12-14-012 data on $\mathrm{Ar}, \mathrm{Ti}$, and $\mathrm{C}$. The $k_{F}$ of $\mathrm{C}$ is fixed to the value obtained by Moniz et al. [84] while the data analysis of $\mathrm{Ti}$ and $\mathrm{Ar}$ sets $k_{F}$ at $240 \mathrm{MeV}$ and $245 \mathrm{MeV}$, respectively. The circles are the Ar data from LNF [45], which turn out to prefer an inconsistently higher value of $k_{F}$.

analysis of the JLab data. This inconsistency may well be the result of the normalization issue that the authors of Ref. [45] found in their ${ }^{16} \mathrm{O}$ cross section as compared to the previously measured cross section at Bates Linear Accelerator Center [74] which was considered as a reference dataset by the authors of Ref. [45]. A normalization factor of 1.19 is then applied to the ${ }^{16} \mathrm{O}$ cross section to reproduce the Bates spectrum, as quoted in Ref. [45]. Note that the Bates data for oxygen was a result of subtracting cross sections obtained for $\mathrm{BeO}$ and $\mathrm{Be}$ 
Chapter 4. First Measurement of the Ar $\left(e, e^{\prime}\right) X$ Cross Section at Jefferson 72

$\mathrm{LAB}$

targets while the LNF oxygen target was a relatively pure jet target. The same normalization factor, 1.19, was then applied to the reported argon cross section leaving room for further disparity. A similar inconsistency is observed also with the analysis of the scaling of the first kind [72] - shown in Fig. 4.4, where the $y$-scaling function, $F(y)$, obtained from the argon, titanium, and carbon cross section measured by the E12-14-012 experiment, and the argon cross section measured at LNF are compared. Additionally, the RGF calculations of Ref. [94] encounter the same normalization issue with the LNF argon data while they successfully describe the LNF data on ${ }^{16} \mathrm{O}$ [94] and the E12-14-012 argon data in the quasielastic region (Fig. 4.1).

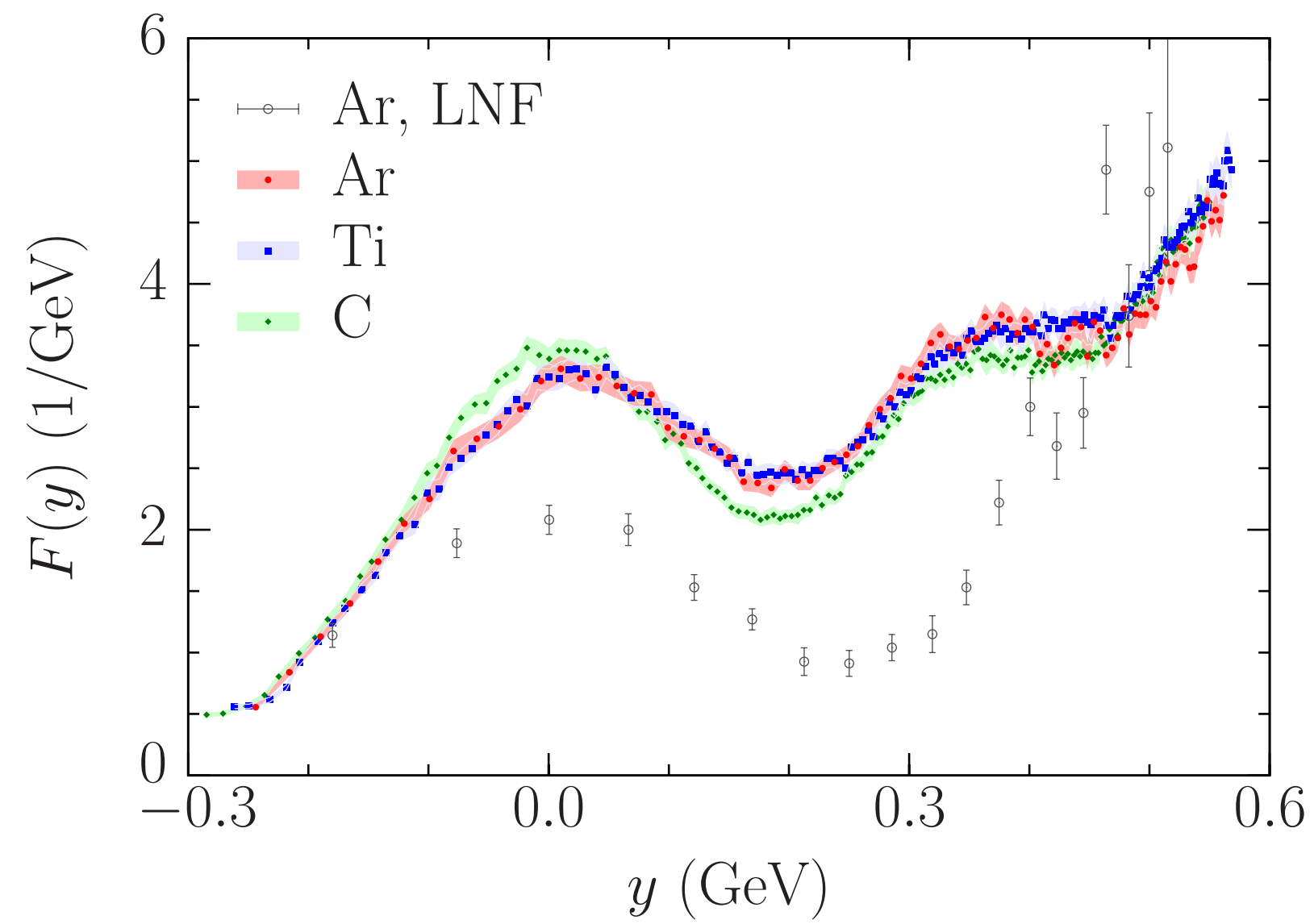

Figure 4.4: Comparison between the scaling function $F(y)$ obtained from the E12-14-012 data on argon, titanium and carbon, and the argon data obtained at LNF [45]. 


\subsection{Summary}

In this Letter, we have reported the first argon results of JLab experiment E12-14-012, as $\operatorname{Ar}\left(e, e^{\prime}\right)$ cross sections at incident electron energy $E=2.222 \mathrm{GeV}$ and scattering angle $\theta=15.541 \mathrm{deg}$. The cross section covers a broad range of energy transfer in which quasielastic scattering and resonance production are the dominant mechanisms of interaction. We presented a comparison of $\operatorname{Ar}\left(e, e^{\prime}\right)$ cross section with previously reported $\operatorname{Ti}\left(e, e^{\prime}\right)$ and $\mathrm{C}\left(e, e^{\prime}\right)$ cross sections of our experiment. The new precise measurement on argon nucleus will be of great value for the development of realistic models of the electroweak response of neutronrich nuclei, vital for the success of the current and next generation of neutrino oscillation studies employing liquid-argon based detectors.

We acknowledge the outstanding support from the Jefferson Lab Hall A technical staff, target group and Accelerator Division. This experiment was made possible by Virginia Tech and the National Science Foundation under CAREER grant No. PHY-1352106. This work was also supported by the DOE Office of Science, Office of Nuclear Physics, contract DE-AC0506OR23177, under which Jefferson Science Associates, LLC operates JLab, DOE contract DE-FG02-96ER40950 and DOE contract DE-AC02-76SF00515. 


\section{Chapter 5}

\section{Measurement of the cross sections for inclusive electron scattering in the E12-14-012 experiment at Jefferson Lab}

The work described in this chapter was published as:

Matt Murphy, Hongxia Dai, Linjie Gu, et al, (The Jefferson Lab Hall A Collaboration), "Measurement of the cross sections for inclusive electron scattering in the E12-14-012 experiment at Jefferson Lab" [138].

Reproduced with permission from the American Physical Society. Copyright (2019) by the American Physical Society.

I contributed the main data analysis work in this paper under Professor Camillo Mariani's supervision. 


\subsection{Introduction}

Electron scattering experiments have been shown to be the best tool for precise investigations of the structure of atomic nuclei [96]. The electromagnetic interaction of electrons with the target is weak compared with the strength of interactions that bind nucleons together, and can be treated as an exchange of a single photon. Allowing the nuclear response to be probed at energy transfers varied independently from momentum transfers, electron beams can be used to investigate physics corresponding to various excitation energies with different spacial resolutions, exposing to different interaction mechanisms.

The existing body of electron-scattering data clearly shows that many important features of nuclear structure can be described assuming that nucleons forming the nucleus behave as independent particles bound in a mean field [97], but this picture is not complete without accounting for correlations between nucleons [98].

While analysis of electron scattering from nuclei is interesting in its own right, accurate description of nuclear effects in interactions of a few-GeV probes is now coming into sharp focus due to its relevance for neutrino physics. As neutrino oscillation parameters are extracted from collected event spectra, and neutrino energies have to be reconstructed from the observed kinematics of the products of their interactions with nuclear targets, nuclear effects play fundamental role in neutrino-oscillation analysis [91].

In neutrino experiments, nuclear models implemented in Monte Carlo (MC) simulations are employed to predict event rate in a near detector, perform its extrapolation to a far detector, estimate the energy carried by undetected particles, and obtain background estimates. While description of nuclear effects is already one of the largest sources of systematic uncertainties in ongoing oscillation studies [99], its importance will increase further in the next generation of oscillation experiments. In particular, to achieve their sensitivity goals, the 
Deep Underground Neutrino Experiment (DUNE) and Hyper-Kamiokande have to reduce uncertainties coming from nuclear cross sections to a few-percent level $[42,100]$.

As weak interactions of neutrinos probe nucleus in a very similar way as electromagnetic interactions of electrons, precise electron scattering data give unique opportunity to validate nuclear models employed in neutrino physics. A theory model unable to reproduce electron measurements cannot be expected to provide accurate predictions for neutrino cross sections.

At the kinematics where the impulse approximation is valid - the process of scattering off a nuclear target can be described as involving predominantly a single nucleon, with $(A-1)$ nucleons acting as a spectator system — nuclear effects can be separated from the description of the elementary cross sections, differing between neutrinos and electrons, and the knowledge gained in electron scattering directly translates to neutrino interactions. In particular, measurements of the $\left(e, e^{\prime} p\right)$ cross sections - in which knocked out protons are detected in coincidence with electrons - can be used to extract the information on the momentum and energy distributions (the spectral function) of protons in the nucleus, and on final-state interactions (FSI) of the struck protons propagating through the (excited) residual nucleus, which are intrinsic properties of the target and do not depend on the interaction mechanism.

In the simplest case of a symmetric nuclear target, with the proton number $Z$ equal to the neutron number $N$, nuclear effects are expected to be largely the same in neutrino and electron interactions, up to small Coulomb corrections. For an asymmetric nucleus, one needs to additionally analyze electron scattering on its mirror nucleus, with $Z$ and $N$ swapped, to obtain a good approximation of information on the neutron structure, impossible to collect directly. In the case of DUNE, in addition to argon $(Z=18, N=22)$ - employed as the target material - it is necessary to collect electron scattering data also for titanium $(Z=22)$. While the exclusive $\left(e, e^{\prime} p\right)$ cross sections give direct insight into the nuclear structure, they do not provide a complete picture of all interaction dynamics. 
When the energy transferred by the interacting electron to the nucleon increases, the interaction mechanism changes from quasielastic (QE) scattering, in which the struck nucleon is removed from the nucleus, to nucleon resonance production, dominated by the excitation of the $\Delta$ resonance, and finally to deep-inelastic scattering on individual quarks forming nucleons. The inclusive $\left(e, e^{\prime}\right)$ measurements, which yield the spectra of electrons scattered at fixed angle, provide information on all interaction mechanisms, regardless of the composition of hadrons in the final state. As a consequence, a great deal can be learned from the inclusive $\left(e, e^{\prime}\right)$ cross sections, particularly in the context of DUNE, in which $\sim 2 / 3$ of events are expected to involve pions [42].

The features of the peaks observed in the inclusive spectrum - their width, position, shape, and height - provide information on the momentum and energy distributions of the nucleons in the nuclear ground state, as well as on the final-state interactions (FSI) between the strucknucleons and the spectator system. The width of the QE peak, which in the nonrelativistic regime depends on both the momentum carried by the struck nucleon and the momentum transfer, $\mathbf{q}$, in the relativistic regime becomes largely independent of $\mathbf{q}$, and can be simply parametrized in terms of a Fermi momentum, $k_{F}$ [84]. However, a kinematic-dependent broadening ascribed to FSI is also observed. The position of the QE peak is determined by the combined effects of nuclear binding and FSI, while its pronounced asymmetry is a consequence of multi-nucleon knockout processes, induced by short-range correlations between nucleons in the initial state and by two-body interaction mechanisms, such as those involving meson-exchange currents. The height of the QE peak depends on the number of nucleons probed by the interaction and the momentum and energy distributions of nucleons in the ground state.

The identification of nuclear effects shaping the peak corresponding to QE scattering largely applies to other interaction mechanisms as well. However, their contributions give rise to 
broader structures in the cross section, as they involve production of hadrons of finite lifetimes.

To provide a reliable foundation for the oscillation analysis of precise long-baseline neutrino experiment, a nuclear model employed in Monte Carlo simulations must be able to reproduce the features of the cross sections for electron scattering corresponding to the kinematics and target of relevance. In the context of DUNE, beam energies between 2 and $4 \mathrm{GeV}$ play the most important role, and argon is the target material.

Previously [92, 101], we have reported the inclusive cross sections for electron scattering off argon, titanium, and carbon, extracted for beam energy $2.222 \mathrm{GeV}$ and scattering angle $15.54^{\circ}$. Here we present a new result for aluminum, and a complete scaling analysis of all the targets that we have analyzed. We also discuss in more details the procedures used to measure the inclusive cross sections, together with their uncertainty estimates. In Sec. 5.2 we describe the setup of the performed experiment. The methods of extracting the cross sections are presented in Sec. 5.3. The estimates of their uncertainties are covered in Sec. 5.4. In Sec. 5.5 we report and discuss the measured aluminum cross section, while Sec. 5.6 is devoted to the scaling analysis of our data. Finally, in Sec. 5.7 we summarize our findings and draw the conclusions.

\subsection{Experimental Setup}

Performed at Jefferson Lab, E12-14-012 took both exclusive electron scattering data $\left(e, e^{\prime} p\right)$ in which the proton knocked out from the nuclear target is detected in coincidence with the scattered electron, and inclusive $\left(e, e^{\prime}\right)$ data in which all final states contribute, for different targets: $\mathrm{C}, \mathrm{Ti}, \mathrm{Ar}$ and $\mathrm{Al}$. The experiment E12-14-012 used an electron beam of energy $2.222 \mathrm{GeV}$ provided by the Continuous Electron Beam Accelerator Facility (CEBAF), 
and took data in Spring 2017. The average beam current was $10 \mu \mathrm{A}$. Scattered electrons were measured using a high resolution spectrometer (HRS), equipped with two vertical drift chambers (VDCs) providing tracking information [33], two scintillator planes for timing measurements and triggering, double-layered lead-glass calorimeter, and a gas Čerenkov counter used for particle identification [15]. The HRS was positioned with a central scattering angle of $\theta=15.54^{\circ}$. The data analysis for inclusive electron scattering is relatively simple, as it implies modest data acquisition (DAQ) rates and very small pion backgrounds. The beam current and position, the latter being critical for the electron-vertex reconstruction and momentum calculation, were monitored by resonant radio-frequency cavities (beam current monitors, or BCMs [15]) and cavities with four antennae (beam position monitors, or BPMs [15]), respectively. The beam size was measured using harp scanners, which moved a thin wire through the beam. The beam was spread over a $2 \times 2 \mathrm{~mm}^{2}$ area to avoid overheating the target.

The experiment employed a set of solid targets - aluminum, carbon (single foil and a multifoil composed of 9 foils), and titanium - as well as a closed cell of gaseous argon [37]. The aluminum target consisted of two identical foils of the 7075 alloy, the thickness of which was $0.889 \pm 0.002 \mathrm{~g} / \mathrm{cm}^{2}$. Details of the elementary composition of the Al-7075 alloy used in the E12-14-012 experiment are given in Table 5.1. The aluminum foils were positioned to match the entrance and exit windows of the argon target, separated by a distance of $25 \mathrm{~cm}$. Their thickness was fixed to be the same as the radiation length of the argon target. The analysis presented here uses the data from one of the foils only, located upstream of the spectrometers at $z=-12.5 \mathrm{~cm}$. The data were taken in nine separate runs, modifying at each step the momentum of the spectrometer in order to cover the final electron energy $E^{\prime}$ from 1.285 to $2.135 \mathrm{GeV}$.

The VDCs' tracking information allowed the determination of the momentum and recon- 
Table 5.1: Composition of the Al-7075 alloy. For each element, we provide the number of protons $Z$ and the average number of neutrons $N$ calculated according to the isotopic abundances [102].

\begin{tabular}{clll} 
& weight $(\%)$ & $\mathrm{Z}$ & $\mathrm{N}$ \\
\hline $\mathrm{Al}$ & 89.72 & 13 & 14.00 \\
$\mathrm{Zn}$ & 5.8 & 30 & 35.45 \\
$\mathrm{Mg}$ & 2.4 & 12 & 12.32 \\
$\mathrm{Cu}$ & 1.5 & 29 & 34.62 \\
$\mathrm{Fe}$ & 0.19 & 26 & 29.91 \\
$\mathrm{Cr}$ & 0.19 & 24 & 28.06 \\
$\mathrm{Si}$ & 0.07 & 14 & 14.11 \\
$\mathrm{Mn}$ & 0.03 & 25 & 30.00 \\
$\mathrm{Ti}$ & 0.03 & 22 & 25.92 \\
$\mathrm{~V}$ & 0.01 & 23 & 28.00 \\
$\mathrm{Zr}$ & 0.01 & 40 & 51.32 \\
other & 0.05 & & \\
\hline average & & $14.26 \pm 0.01$ & $15.58 \pm 0.01$
\end{tabular}

struction of the direction (in-plane and out-of-plane angles) of the scattered electron, and reconstructing the interaction vertex at the target. The transformation between focal plane and target quantities was computed using an optical matrix, the accuracy of which was verified using the multi-foil target data. Possible variations of the magnetic field in the HRS magnets that could affect the optics are included in the analysis as systematic uncertainties.

We set up two types of hardware triggers:

$$
\begin{aligned}
& T_{3}=\left(S_{0} \& \& S_{2}\right) \& \&(L C \| G C), \\
& T_{5}=\left(S_{0} \| S_{2}\right) \& \&(L C \| G C) .
\end{aligned}
$$

The $T_{3}\left(T_{5}\right)$ trigger type requires that the signal from the scintillator plane $S_{0}$ AND $S_{2}\left(S_{0}\right.$ OR $\left.S_{2}\right)$ is detected in coincidence with the signal from the lead calorimeter $(L C)$ or the gas Čerenkov counter $(G C)$. 
Electrons were selected in the HRS requiring, in addition, one reconstructed track. Further, they had to deposit $30 \%$ of their energy in the lead calorimeter $\left(E_{\text {cal }} / p>0.3\right)$. To select events in the central acceptance region of the spectrometer, the electron's track was required to be within $\pm 4 \mathrm{mrad}$ of the in-plane angle and $\pm 6 \mathrm{mrad}$ of the out-of-plane angle with respect to the center ray of the spectrometer and have a $d p / p$ of \pm 0.04 . The spectrometers were calibrated using sieve slit measurements and the position of the spectrometers and angles were surveyed before the start of the data taking. The survey precision for the angle and position measurements is respectively $0.01 \mathrm{mrad}$ and $0.01 \mathrm{~mm}$.

The efficiencies of the elements in the detector stack were studied by comparing rates in various combinations of secondary triggers. The scintillator efficiency, $\left(S_{0} \& \& S_{2}\right)$, was studied using the ratio of the events rates selected using $T_{3}$ and $T_{5}$ trigger types, requiring one reconstructed track, and applying the acceptance and calorimeter cuts. It was found to be $99 \%$. The efficiency of the calorimeters was close to $100 \%$ for all runs, the efficiency of the Črenkov detector was found to range from $99.9 \%$ for the highest $E^{\prime}$ runs down to $97.5 \%$ for the lowest $E^{\prime}$ run. The Čerenkov efficiency was evaluated by selecting a pure sample of electrons in the calorimeter and varying the Cerenkov cut by $\pm 10 \%$. The livetime of the electronics, computed using the rates from scalers, which were independent of triggered events, was above $98 \%$ for all runs. The acceptance cuts efficiencies ( $98 \%)$ and the $d p$ cut efficiency ( $\sim 99 \%)$ were computed using the MC simulation [68]. The overall efficiency (between 88 and 95\% across all the kinematical regions) includes cuts on the calorimeters, both the lead and the Čerenkov counter, track reconstruction efficiency, livetime and acceptance. 
Chapter 5. Measurement of the Cross sections for inclusive electron

\subsection{Data Analysis}

\subsubsection{Yield-Ratio Method}

The yield-ratio method of determining the cross section involves both the experimental data and simulated MC data. In this method, the yield $Y$ is calculated for both sets of data as

$$
Y^{i}=\left(N_{S}^{i} \times P S\right) /(L T \times \epsilon),
$$

where $i$ refers to the $i$ th bin of the $E^{\prime}$ distribution, $N_{S}^{i}$ is the total number of scattered electrons, $P S$ is a pre-scale factor in the DAQ, $\epsilon$ is the total efficiency of the detector, and $L T$ is the livetime of the electronics. The ratio of the yields for the actual and MC data is taken as a means of eliminating any impact of the acceptance on each bin, and then the measured cross section is determined by multiplying this ratio by the cross section calculated within the Monte Carlo model:

$$
\frac{d^{2} \sigma_{\mathrm{data}}}{d \Omega d E^{\prime}}=\frac{d^{2} \sigma_{\mathrm{MC}}}{d \Omega d E^{\prime}} \times \frac{Y_{\mathrm{data}}}{Y_{\mathrm{MC}}} .
$$

\subsubsection{Acceptance Method}

The cross section was also extracted via another method, the acceptance method, and both the yield ratio and acceptance methods were examined for agreement. In the case of the argon target, for which an accurate model of the nuclear response is not yet available, it is important to validate the MC simulation and results obtained using the yield ratio method using an alternative method less dependent on the input MC cross section model. The acceptance method will be described in the following of this section. 
For each $(\Delta E, \Delta \Omega)$ bin, the number of detected electrons can be determined using

$$
N_{S}^{i}=L \times \frac{d^{2} \sigma}{d \Omega d E^{\prime}} \times \Delta E^{\prime} \Delta \Omega \times \epsilon \times A^{i}\left(E^{\prime}, \theta, \phi\right)
$$

where $L$ is the integrated luminosity (number of beam electrons $\times$ number of targets / area), $\epsilon$ is the total detection efficiency, and $\theta$ and $\phi$ represent the in-plane and out-of-plane angles, respectively. The acceptance $A^{i}\left(E^{\prime}, \theta, \phi\right)$ is the probability that a particle passes through the spectrometer into the $i$ th bin.

The electron yield corrected for the overall efficiency (product of individual efficiencies as described above) can be cast as

$$
\begin{aligned}
Y^{i} & =\frac{N_{S}^{i}}{\epsilon} \\
& =L \times \frac{d^{2} \sigma_{\text {data }}}{d \Omega d E^{\prime}} \times \Delta E^{\prime} \Delta \Omega \times A^{i}\left(E^{\prime}, \theta, \phi\right),
\end{aligned}
$$

and the cross section can be measured using

$$
\frac{d^{2} \sigma_{\mathrm{data}}}{d \Omega d E^{\prime}}=\frac{Y^{i}}{\Delta E^{\prime} \Delta \Omega \times A^{i}\left(E^{\prime}, \theta, \phi\right) \times L} .
$$

The single-arm Monte Carlo simulation was used to generate events uniformly distributed in $\left(\theta, \phi, E^{\prime}\right)$. For a specific phase-space slice in $\left(\Delta \theta, \Delta \phi, \Delta E^{\prime}\right)$, we computed the ratio between the total number of events that reach the spectrometer and the number of generated events. The ratio of these two numbers represents the probability that a particle successfully passes through the magnets and the aperture to arrive at the detector package.

For an extended target, an acceptance matrix $A^{i}\left(E^{\prime}, \theta, \phi\right)$ was generated at various points along the target length. Each different target slice was associated with a different $A^{i}\left(E^{\prime}, \theta, \phi\right)$. The number and size of the slices were optimized based on the statistics of the data. In 
principle, an infinite number of matrices could be used in order to make events perfectly weighted, but this method would be inefficient and subject to large statistical fluctuations, if the number of events in each region was limited.

In this analysis, we used a single matrix for events along the entire target length to correct the data, and evaluated the residual variation along the beam direction $z$. For these studies we took advantage of the optical target data, collected in Spring 2017.

The optical target was a series of nine carbon foils, placed along the beam direction at $z=0 \mathrm{~cm}, \pm 2 \mathrm{~cm}, \pm 5 \mathrm{~cm}, \pm 7.5 \mathrm{~cm}, \pm 10 \mathrm{~cm}$, respectively. The $z$ distribution of the events reconstructed from the optical target is shown in Fig. 5.1, with the shaded regions representing the $z$-position cuts employed to identify the events coming from individual carbon foils. Because it would be difficult to select pure events from each foil, due to its finite thickness, we used the Monte Carlo simulation and the carbon cross-section model to generate single-foil carbon data for different $z$ positions of the target.

Using the single-foil carbon data, we generated 9 acceptance matrices corresponding to the mean $z$ position of each foil composing the multi-foil carbon target. We applied a weight of $1 / A\left(E^{\prime}, \theta, \phi\right)$ to every event, and made a comparison between the events originating from individual foils. The obtained distribution of MC event yields from different foils, normalized to the one from the foil at $z=0 \mathrm{~cm}$, is shown in Fig. 5.2. The results for the 9 regions, represented by the red dots lying inside the green shaded band, are in excellent agreement, with variations between them remaining below $0.5 \%$.

When the same procedure is applied to the reconstructed data events, the obtained event yields - represented by the blue lozenges in Fig. 5.2 - exhibit a dependence on the target $z$ position. This behavior is expected due to the variation of the cross section as a function of the electron scattering angle, as foils at different positions have different acceptances, 


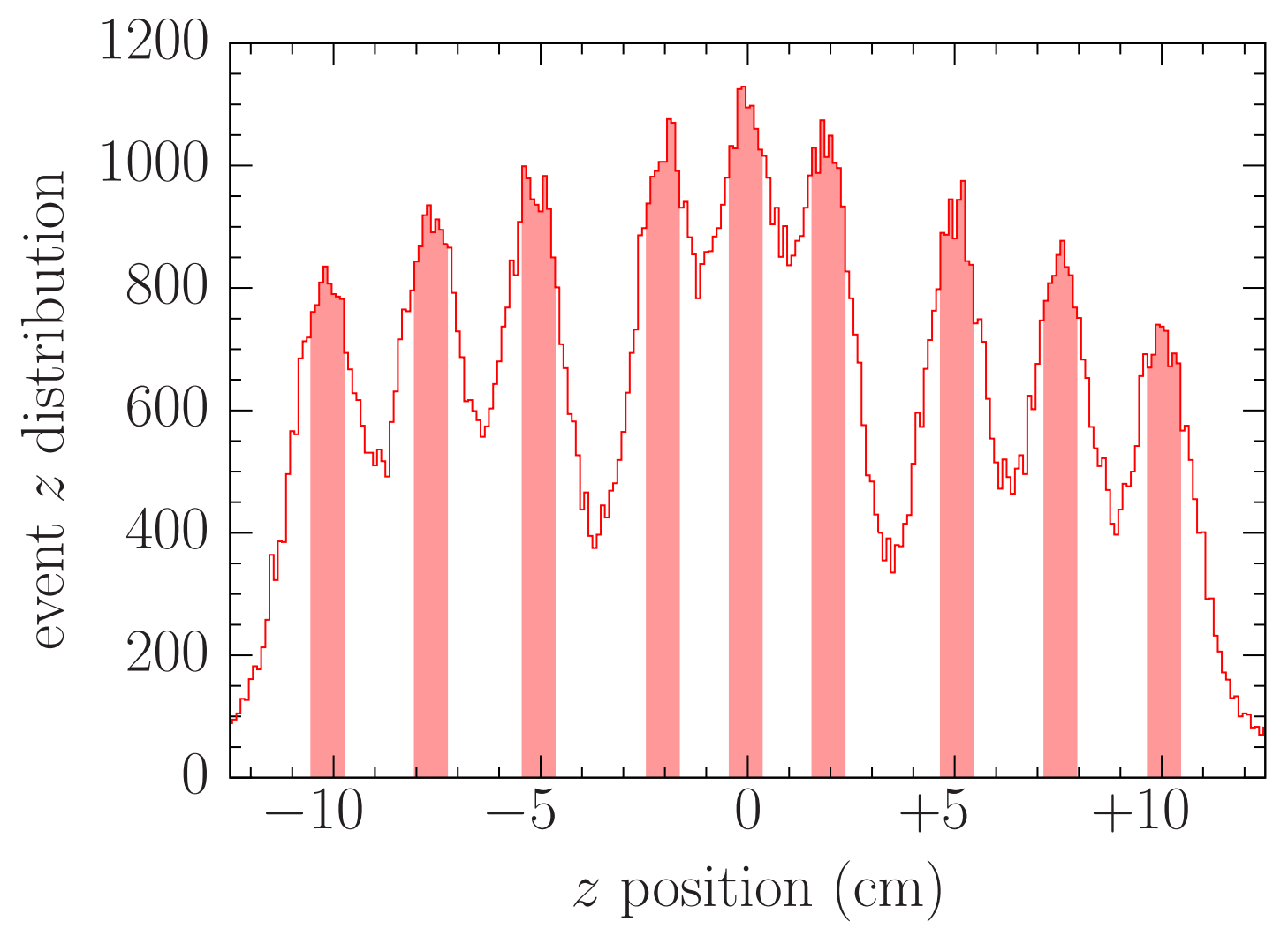

Figure 5.1: Distribution along the beam direction of reconstructed events for the multi-foil carbon target. The shaded regions represent the data selected to identify the events coming from individual carbon foils. 
Chapter 5. Measurement of the Cross SeCtions For inClusive electron SCATTERING IN THE E12-14-012 EXPERIMENT AT JEFFERSON LAB

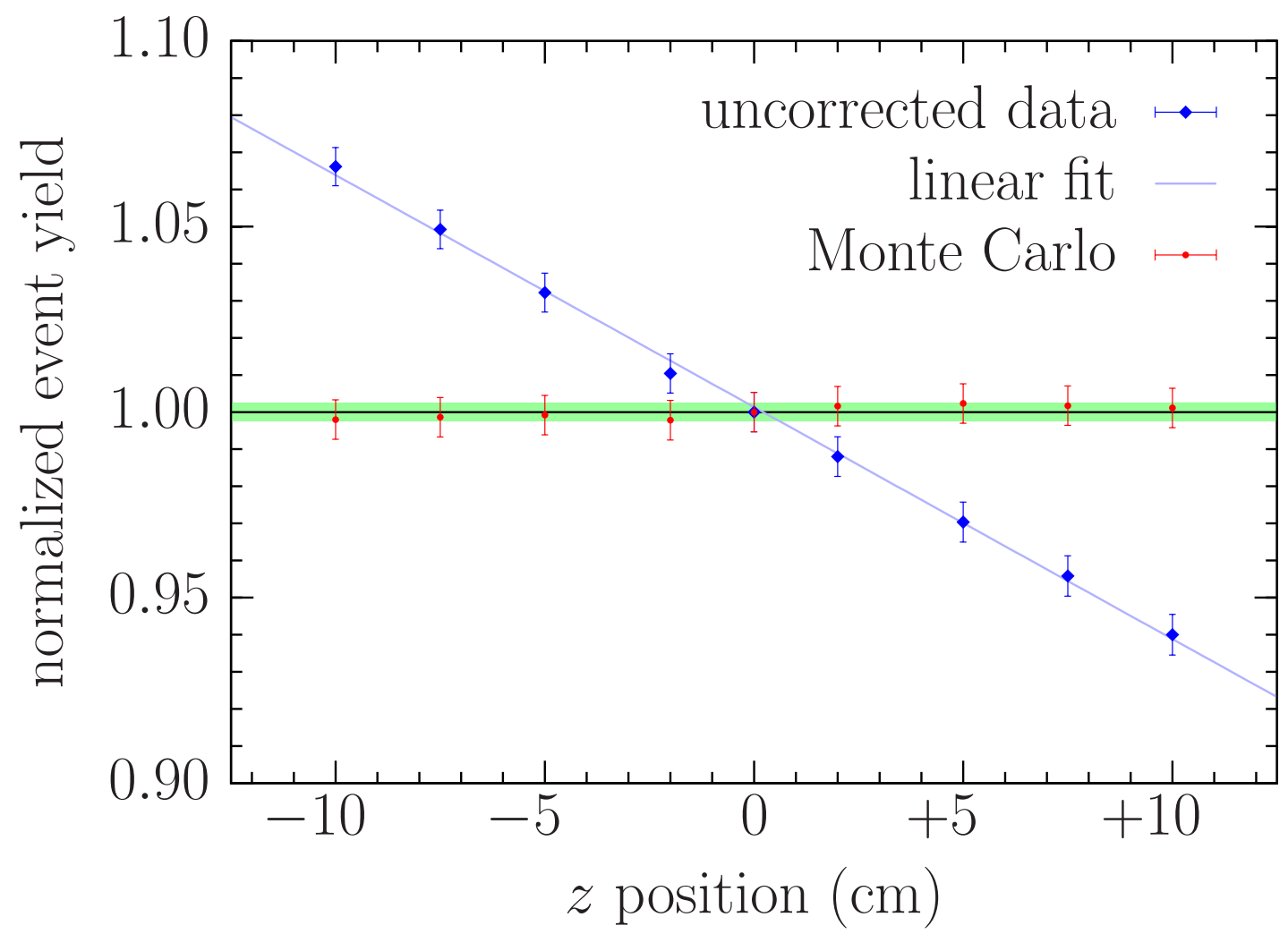

Figure 5.2: Event yields from carbon foils at different positions along the beam direction, normalized to the yield for the central foil, for the uncorrected data and the Monte Carlo simulation. The dependence of the cross section on the scattering angle, correctly taken into account in the Monte Carlo simulation, introduces a linear trend in the data that needs to be corrected for. All uncertainties are purely statistical. 


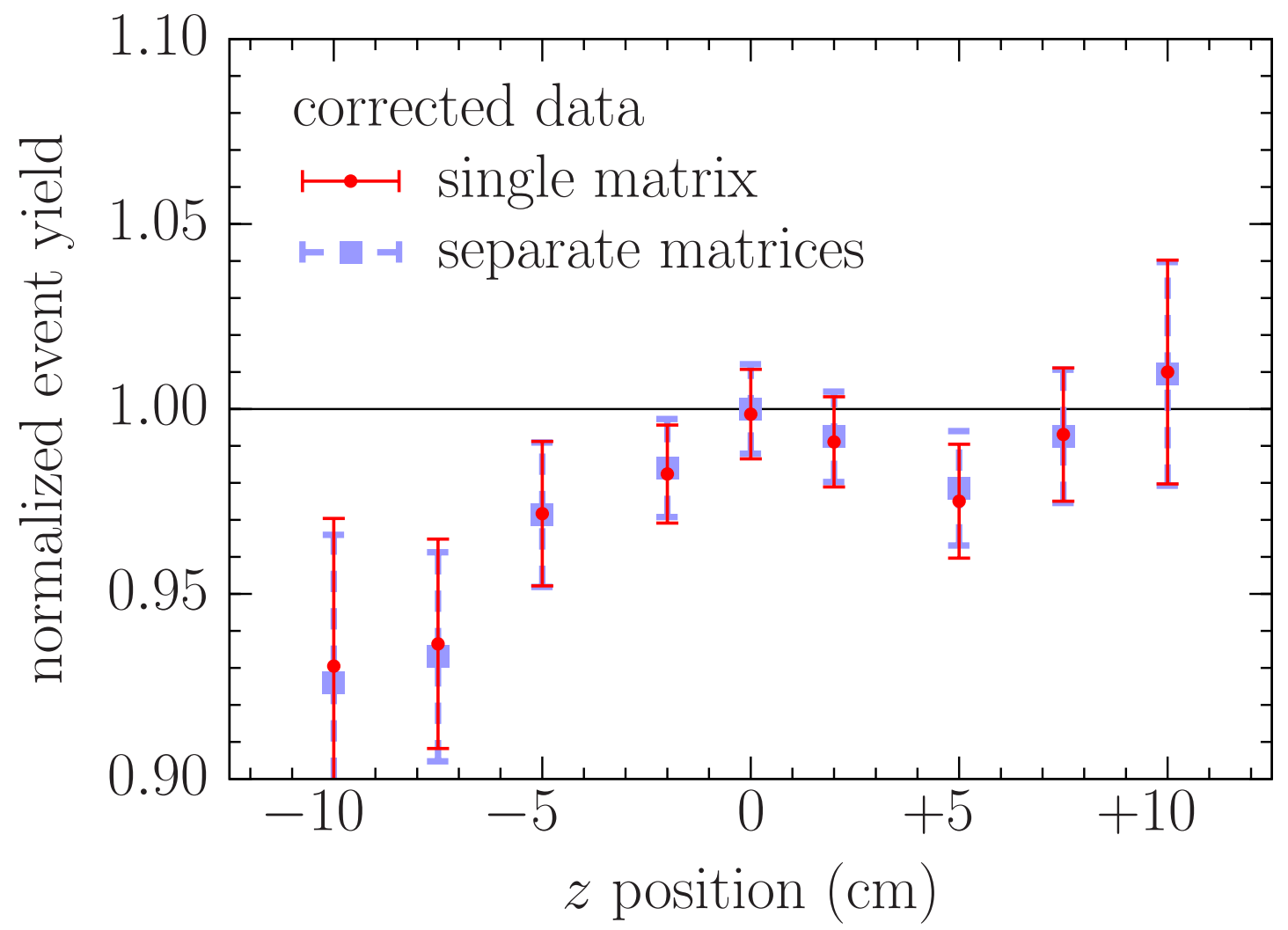

Figure 5.3: Event yields in the corrected data for the multi-foil carbon target surviving the $z$-position selection, normalized to the yield for the central foil. The outcomes of two correction procedures are compared. The error bars are symmetric and represent the total uncertainties, being the statistical and systematic uncertainties added in quadrature. 
depending on the mean value of the scattering angle. The observed $z$ dependence of the event yields is in a good agreement with a linear function $\left(\chi^{2} / \mathrm{NDF}=0.35\right)$ and a correction is applied to the data. Note that this behavior is absent in the MC event yields (the red dots in Fig. 5.2), because the MC simulation takes into account differences in the acceptance for individual foils. In the data analysis, we relied on the reconstructed target $z$ position to identify events coming from each of the 9 carbon foils, applying the selections represented by the shaded regions in Fig. 5.1. The selected events were then corrected using the acceptance matrix computed at $z=0 \mathrm{~cm}$. In order to determine the sensitivity to this approximation, we repeated the same study using 9 different matrices (one for each carbon foil) and found a negligible variation, as shown in Fig 5.3. The obtained event yields are subject to the systematic uncertainties due to the $z$-position selection applied to identify events coming from individual foils.

As a final remark, we note that to acquire the inclusive data, we varied the momentum settings of the left-arm spectrometer in the MC to determine its effect on the acceptance matrix, and found that it is negligible.

\subsection{Uncertainty Analysis}

The total systematic uncertainty in this analysis was estimated by adding in quadrature the contributions listed in Table 5.2. Each of the uncertainties was considered completely uncorrelated. We determined the cuts ensuring that there are no dependencies on kinematical variables and, therefore, all the uncertainties affects only the normalization of the extracted cross sections. The kinematical cuts used in the analysis were varied by $\pm 10 \%$ or by the resolution of the variable under consideration.

As the obtained results depend on the Monte Carlo calculation, it is important to estimate 
Table 5.2: Contributions to systematic uncertainties in the yield-ratio method for aluminum and argon.

\begin{tabular}{lcc} 
& $\mathrm{Al}$ & $\mathrm{Ar}$ \\
\hline a. Beam energy & $0.1 \%$ & $0.1 \%$ \\
b. Beam charge & $0.3 \%$ & $0.3 \%$ \\
c. Beam $x$ offset & $<1.0 \%$ & $<0.8 \%$ \\
d. Beam $y$ offset & $<1.0 \%$ & $<0.9 \%$ \\
e. HRS $x$ offset & $<0.8 \%$ & $<1.0 \%$ \\
f. HRS $y$ offset & $<0.6 \%$ & $<0.8 \%$ \\
g. Optics (q1, q2, q3) & $<1.8 \%$ & $<1.0 \%$ \\
h. Target thickness & $0.2 \%$ & $0.7 \%$ \\
i. Acceptance cut $(\theta, \phi, d p / p)$ & $<1.0 \%$ & $<2.4 \%$ \\
(i) $d p$ acceptance cut & $<0.32 \%$ & - \\
(ii) $\theta$ acceptance cut & $<0.32 \%$ & - \\
(iii) $\phi$ acceptance cut & $<0.79 \%$ & - \\
(iv) $z$ acceptance cut & $<0.45 \%$ & - \\
j. Calorimeter cut & $<0.02 \%$ & $<0.02 \%$ \\
k. Crerenkov cut & $<0.12 \%$ & $<0.07 \%$ \\
l. Cross section model & $<0.2 \%$ & $<1.3 \%$ \\
m. Radiative and Coulomb corr. & $1.0 \%-1.3 \%$ & $1.0 \%-1.3 \%$ \\
\hline Total systematic uncertainty & $1.7 \%-2.7 \%$ & $1.8 \%-3.0 \%$ \\
& &
\end{tabular}

uncertainties resulting from its inputs. To determine the uncertainties related to the target position, we performed the simulation with the inputs for the beam's and spectrometer's $x$ and $y$ offsets varied within uncertainties, and we recomputed the optical transport matrix varying the three quadrupole magnetic fields, one at the time. Each of these runs was compared to the reference run, and the corresponding differences were summed in quadrature to give the total systematic uncertainty due to the Monte Carlo. That summed uncertainty value varied from 1.1 to $2.2 \%$, based on the momentum setting for each of the run, and was the largest single source of systematic error.

The systematic uncertainty due to the cuts on the calorimeter and Čerenkov detector was calculated in a similar way, by varying the cut by a small amount and calculating the 
difference with respect to the nominal cut. Given the already high efficiency of these cuts, this resulted in a very small contribution to the uncertainty. The uncertainty due to the acceptance cuts on the angles and on $d p / p$ was calculated in the same way. We included a fixed uncertainty relative to the beam charge and beam energy as in previous work on $\mathrm{C}$ and Ti [92].

The measured cross section is also corrected for the effects from internal and external radiative processes. Internal radiative process are vacuum polarization, vertex corrections, and internal bremsstrahlung. External radiative processes refer to electrons losing energy while passing through material in the target. We applied the radiative correction following the recipe of Dasu [23], using the approach of Mo and Tsai [22], which is subject to theoretical uncertainties and depends on the cross-section model. We consider a fixed 1\% uncertainty due to the theoretical model for the radiative corrections over the full kinematical range. To account for the cross-section model dependence - the same for both the yield-ratio and acceptance methods - we added an additional uncertainty (fully uncorrelated), estimated by computing the difference in the final double differential cross section when the cross section model is rescaled by $\sqrt{\left(Q^{2}\right)} / 2, Q^{2}$ being the four-momentum transfer squared. Coulomb corrections were included in the local effective momentum approximation, following Ref. [70]. A $10 \%$ uncertainty associated with the Coulomb potential was included as systematic uncertainty.

Near the quasielastic peak, there is a non-negligible contribution of the elastic cross section to the inclusive cross section, through the radiative processes. To estimate the corresponding uncertainty, we increased the tail of the elastic contribution by $20 \%$, recalculated the radiative correction, and used its difference with respect to the reference correction as an estimate of the corresponding systematic uncertainty. Finally, we included a target thickness uncertainty. 


\subsection{Experimental Results}

The cross section for inclusive scattering of electrons on the Al-7075 target, extracted using the yield-ratio method and normalized per nucleus, is shown in Fig. 5.4. Its total uncertainties - represented by the outer bands - are obtained by adding in quadrature statistical and systematic uncertainties. As in the case of the previously reported results [92, 101], the aluminum measurement is very precise and limited by the systematic uncertainties.

As a cross check, we also extracted the Al cross section using the acceptance method. Figure 5.5 shows that the results obtained using the two methods are in good agreement. Note that in the acceptance method we did not estimate systematic uncertainties, the error bars

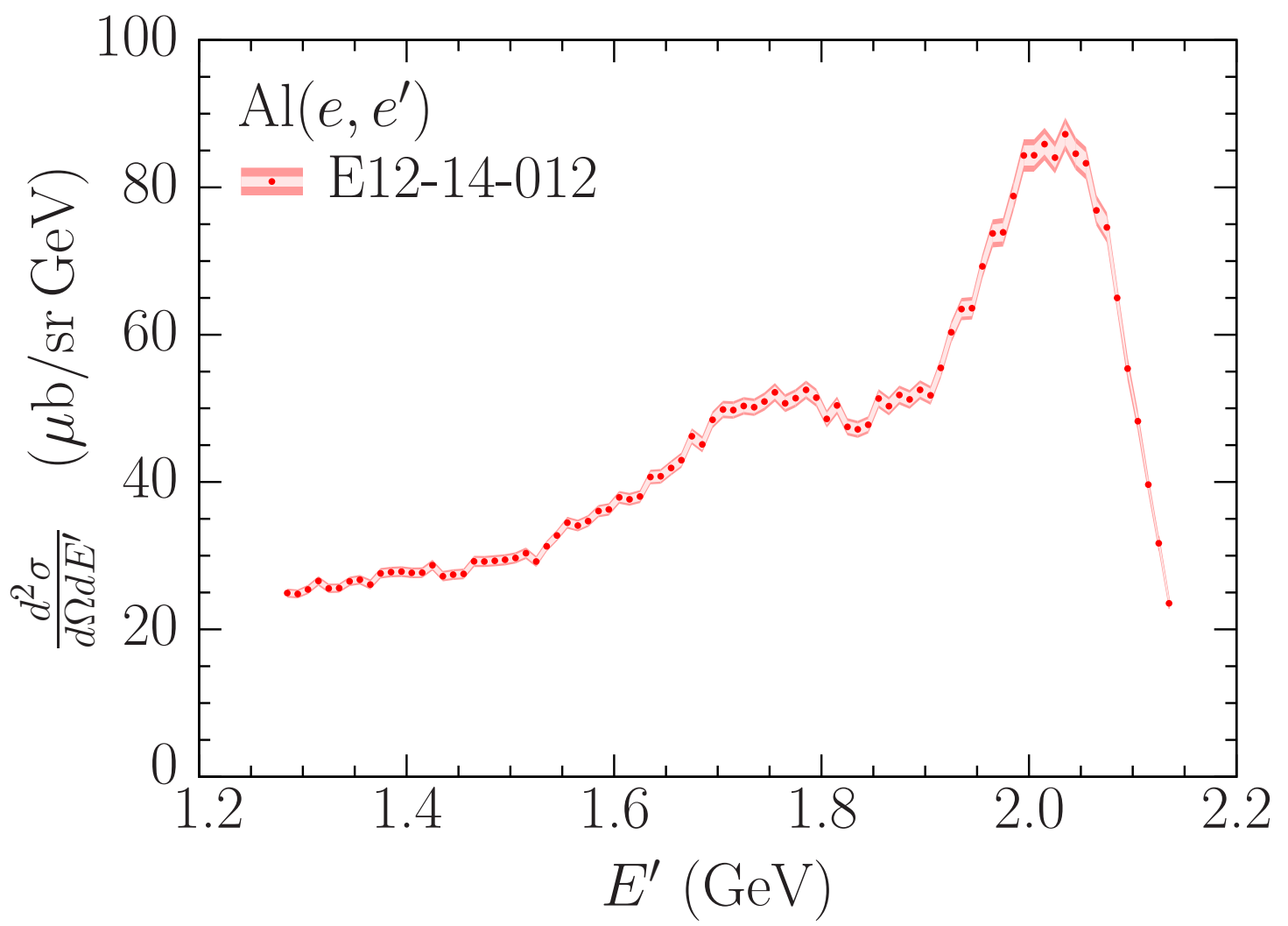

Figure 5.4: Double-differential cross section extracted for inclusive electron scattering off the Al-7075 target at beam energy $2.222 \mathrm{GeV}$ and scattering angle $15.54^{\circ}$. The inner and outer uncertainty bands correspond to statistical and total uncertainties, respectively. 
Chapter 5. Measurement of The CROSS SECTIONS FOR INClusive EleCtron SCATTERING IN THE E12-14-012 EXPERIMENT AT JEFFERSON LAB

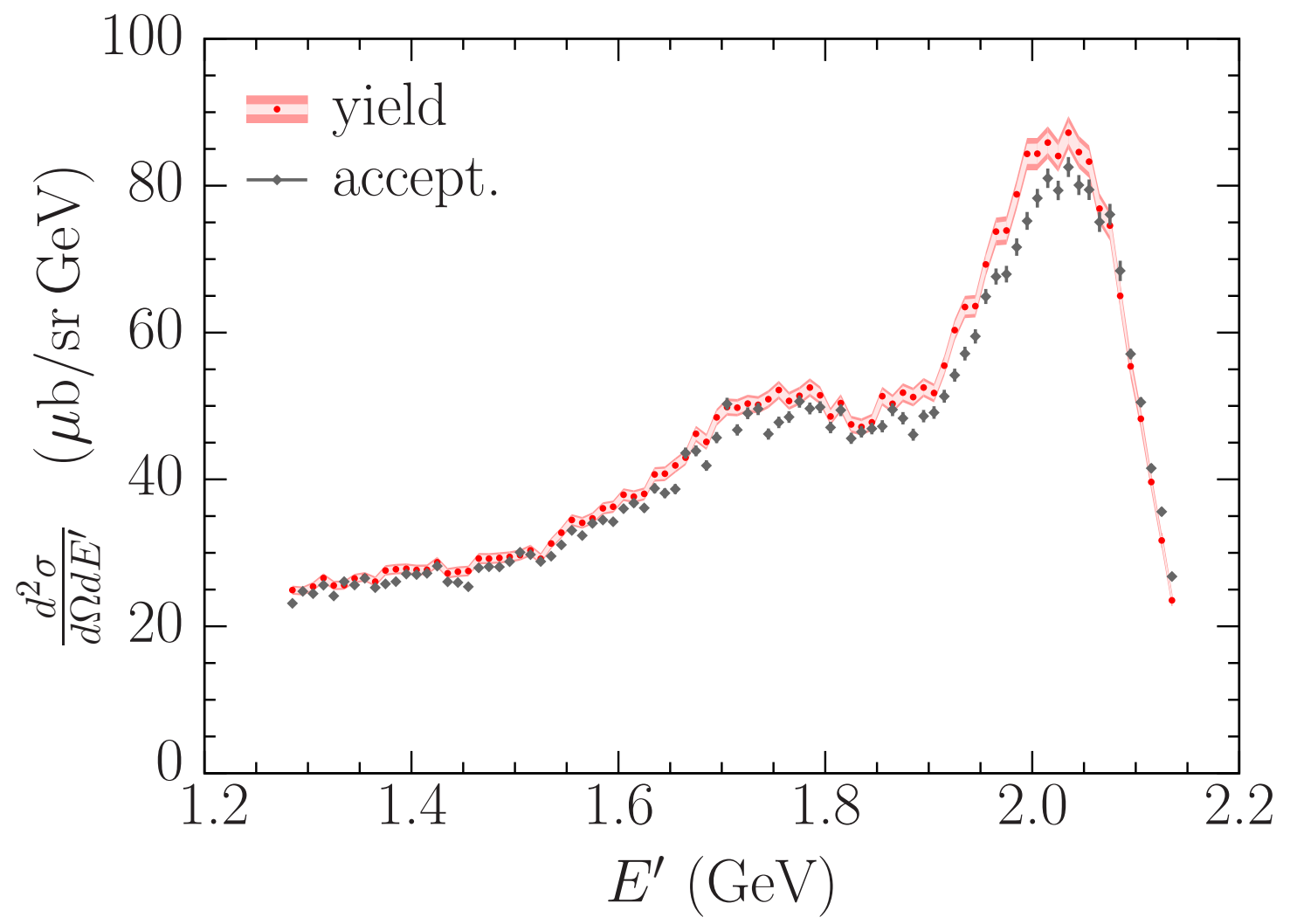

Figure 5.5: Comparison of the $\mathrm{Al}\left(e, e^{\prime}\right)$ cross sections extracted using the yield-ratio and acceptance methods. The inner (outer) bands for the yield-method show statistical (total) uncertainties. For the acceptance method only statistical uncertainties are shown. 
represent the statistical uncertainties only.

The agreement between the yield-ratio and acceptance results was observed for the carbon, aluminum, titanium, and argon targets and provides validation for the approximation employed in the acceptance method, namely using a single acceptance matrix computed at $z=0 \mathrm{~cm}$, as explained in Sec. 5.3.2. The consistency between the yield and acceptance methods for all analyzed targets also indicates that the yield-ratio result exhibits only weak dependence on the input cross section used in the Monte Carlo simulation to correct the data for efficiency and acceptance. This issue is particularly important in the case of the titanium and argon targets, where the cross-section simulations cannot be validated against existing data. Note that the radiative corrections applied in both methods are the same and do depend on the input cross section. The related uncertainties are discussed in Sec. 5.4.

To illustrate how nuclear effects affect different interaction channels, in Fig. 5.6 we compare the per-nucleon cross sections for aluminum, argon, titanium, and carbon. While for every target we account for the abundances of naturally occurring isotopes [102], this effect is relevant only for the $\mathrm{Al}$ target. It is a consequence of the non-negligible contributions of elements heavier than ${ }_{13}^{27} \mathrm{Al}$ to the Al-7075 alloy, detailed in Table 5.1. At the considered kinematics, corresponding to the beam energy $2.222 \mathrm{GeV}$ and scattering angle $15.54^{\circ}$, the cross sections per nucleon for targets ranging from carbon $(A=12.01)$ to titanium $(A=$ 47.92) turn out to be in very good agreement in the region where different pion production mechanisms dominate. While this finding is by no means obvious - due to asymmetry of the proton and neutron numbers for aluminum, argon, and titanium - it is consistent with the results of Refs. $[74,75]$ at energies $\sim 0.54-1.50 \mathrm{GeV}$ and scattering angles $\sim 37^{\circ}$.

The influence of nuclear effects on QE interactions can be better illustrated in terms of the cross sections normalized to the elementary contributions of neutrons and protons that 
Chapter 5. Measurement of the Cross sections for inclusive electron

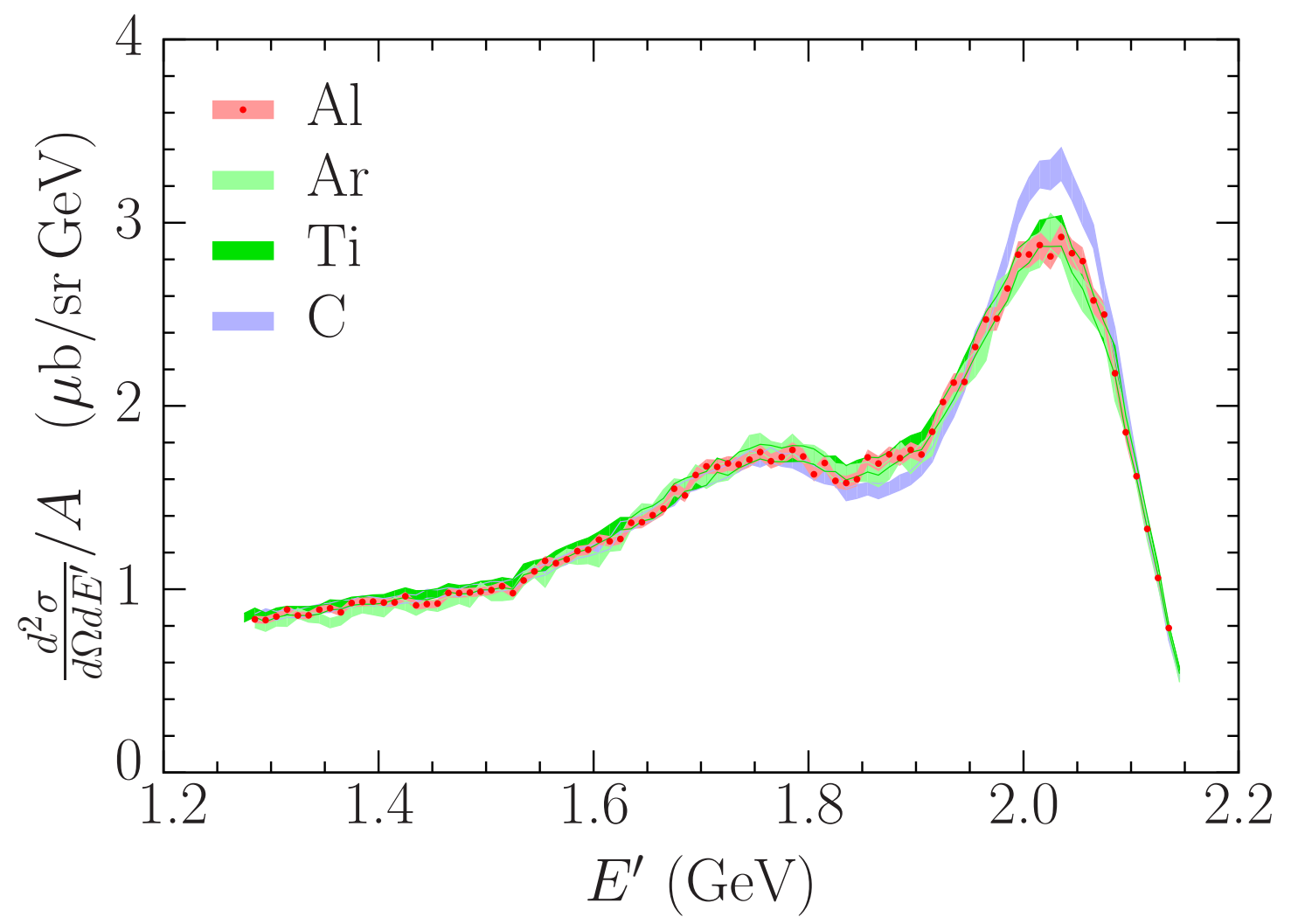

Figure 5.6: Comparison of the cross sections per nucleon for aluminum, argon [101], titanium [92], and carbon [92] measured at beam energy $2.222 \mathrm{GeV}$ and scattering angle $15.54^{\circ}$. The average nucleon number for every target is calculated according to the natural abundances of isotopes, see details in the text. The bands represent the total uncertainties. 


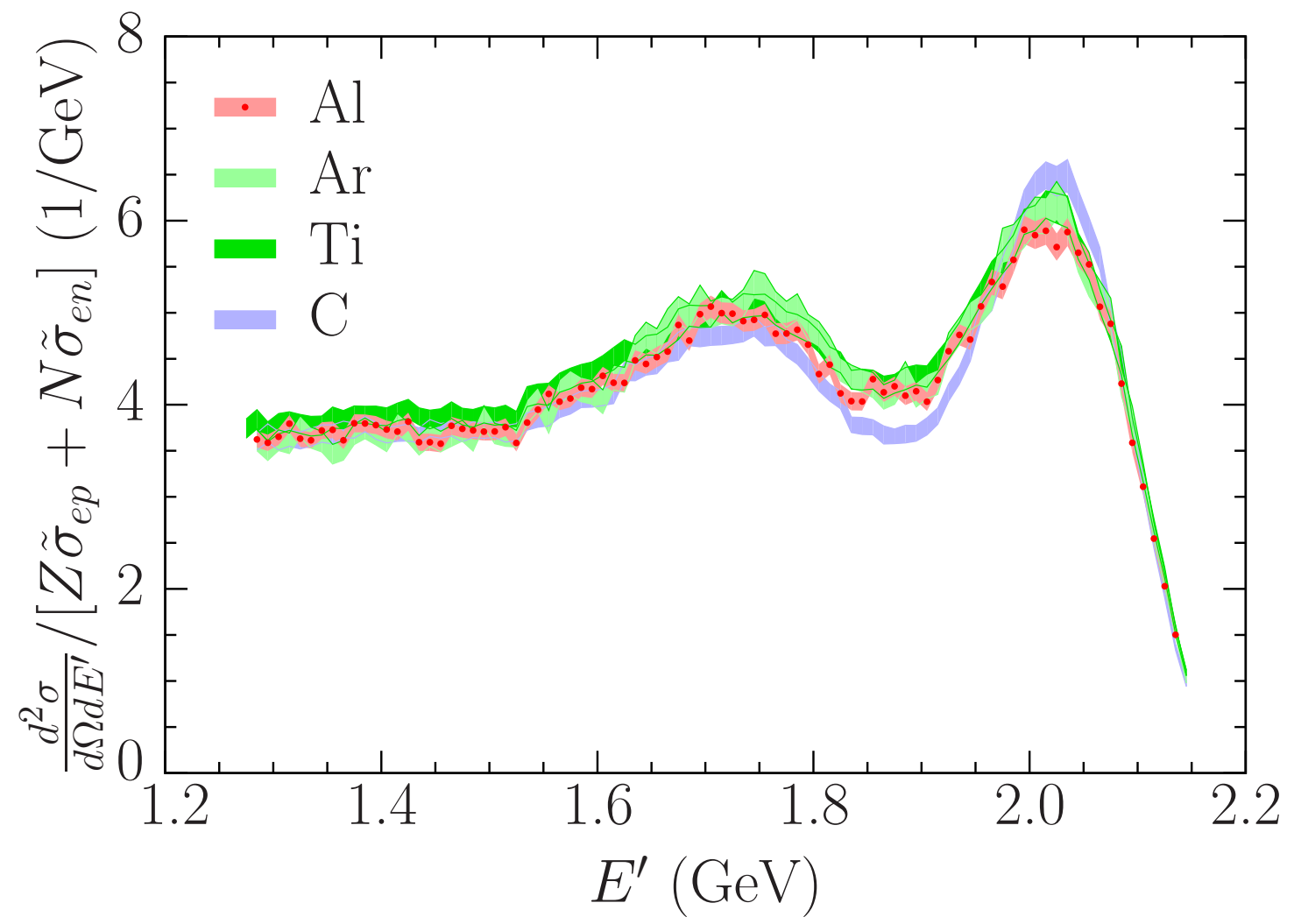

Figure 5.7: Same as in Fig. 5.6 but for the cross sections normalized by the combination of the elementary cross sections according to Eq. (5.6). 
compose the nucleus, that is the quantity

$$
\frac{d^{2} \sigma}{d \Omega d E^{\prime}} /\left[Z \tilde{\sigma}_{e p}+N \tilde{\sigma}_{e n}\right]
$$

where $Z$ and $N$ are the proton and neutron numbers, respectively, while $\tilde{\sigma}_{e p}$ and $\tilde{\sigma}_{e n}$ denote the elastic electron-proton and electron-neutron cross sections stripped of the energyconserving delta function [95]. In the following, we use the average neutron numbers calculated according to the natural abundances of isotopes, that is 6.01 for carbon, 21.98 for argon, and 25.92 for titanium [102]. For the aluminum target, we employ $Z=14.26$ and $N=15.58$, due to its composition listed in Table 5.1.

As shown in Fig. 5.7, the results for titanium and argon are, within uncertainties, identical in the QE peak, but they differ from both those for carbon and aluminum. Near the maximum of the QE peak, the cross section defined in Eq. (5.6) is lower by $\sim 4 \%$ for aluminum, and higher by $\sim 5 \%$ for carbon, than the ones for argon and titanium. In the dip region, the results for aluminum (carbon) are lower by $\sim 2 \%(\sim 13 \%)$ compared with those for argon and titanium.

In QE scattering, the cross sections normalized according to Eq. (5.6) exhibit very weak target dependence only in the region of high $E^{\prime}$, corresponding to low energy transfers, as shown in Fig. 5.7. This is, however, not the case in the QE peak's maximum and for lower $E^{\prime}$, where the energy transferred by electrons to the nucleus is sufficiently high to probe deeply bound states and also to induce two-nucleon knockout.

The observed differences in the dependence on the atomic number of various interaction mechanisms - previously reported in Refs. $[75,103,104]$ — can be expected to provide important clues for building models of nuclear effects valid over broad kinematic regimes and able to describe a range of targets. Such models are of great importance to long-baseline 
neutrino-oscillation experiments.

\subsection{Scaling and $A$-dependence}

The scaling analysis allows to compare inclusive electron-scattering data taken in different kinematical conditions and using different targets.

Scaling of first kind, or $y$-scaling, is observed in the kinematical region of large momentum transfer, $|\mathbf{q}|$, and energy transfer $\omega<\sqrt{|\mathbf{q}|^{2}+m^{2}}-m$, in which the beam particle interacts with individual nucleons and the dominant reaction mechanism is quasielastic scattering $[72,73]$. Under these conditions, the target response, which in general depends on both momentum and energy transfers, reduces to a function of the single variable $y=y(|\mathbf{q}|, \omega)$, defined by the equation

$$
\begin{aligned}
\omega+M_{A}= & \sqrt{y^{2}+\left(M_{A}-m+E_{\min }\right)^{2}} \\
& +\sqrt{(y+|\mathbf{q}|)^{2}+m^{2}} .
\end{aligned}
$$

Here, $m$ and $M_{A}$ are the nucleon mass and the mass of the target nucleus, respectively, while $E_{\min }$ denotes the nucleon knockout threshold. The scaling variable $y$, having the dimension of energy, is simply related to the longitudinal component of the initial momentum of the struck nucleon, $k_{\|}=\mathbf{k} \cdot \mathbf{q} /|\mathbf{k}|$. The scaling function $F(y)$ is determined from the measured cross section, $\sigma^{\exp }$ through

$$
F(y)=K \frac{\sigma^{\exp }}{Z \tilde{\sigma}_{e p}+N \tilde{\sigma}_{e n}},
$$

with $K$ a kinematic factor.

In Fig. 5.8, the $y$-scaling function of aluminum, computed using the cross section displayed in Fig. 5.4 and the average proton and neutron numbers from Table 5.1, is compared to those 
Chapter 5. Measurement of the Cross SeCtions for inClusive electron SCATTERING IN THE E12-14-012 EXPERIMENT AT JEFFERSON LAB

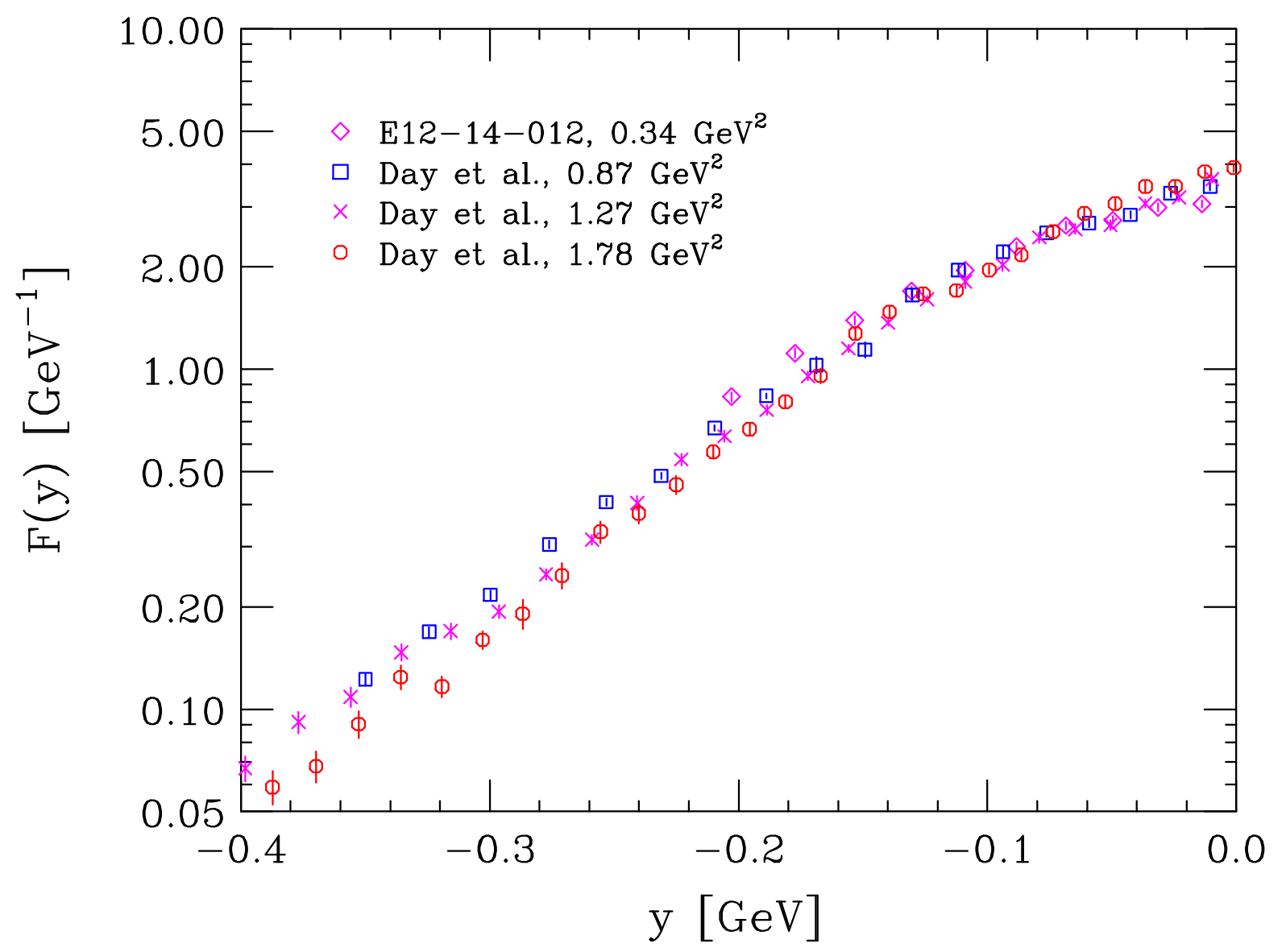

Figure 5.8: Comparison between the scaling function of aluminum obtained from the E1214-012 data (this work), represented by diamonds, and those obtained from the data of Day et al. [76]. The data are labeled according to the value of $Q^{2}$ corresponding to quasi elastic kinematics. 


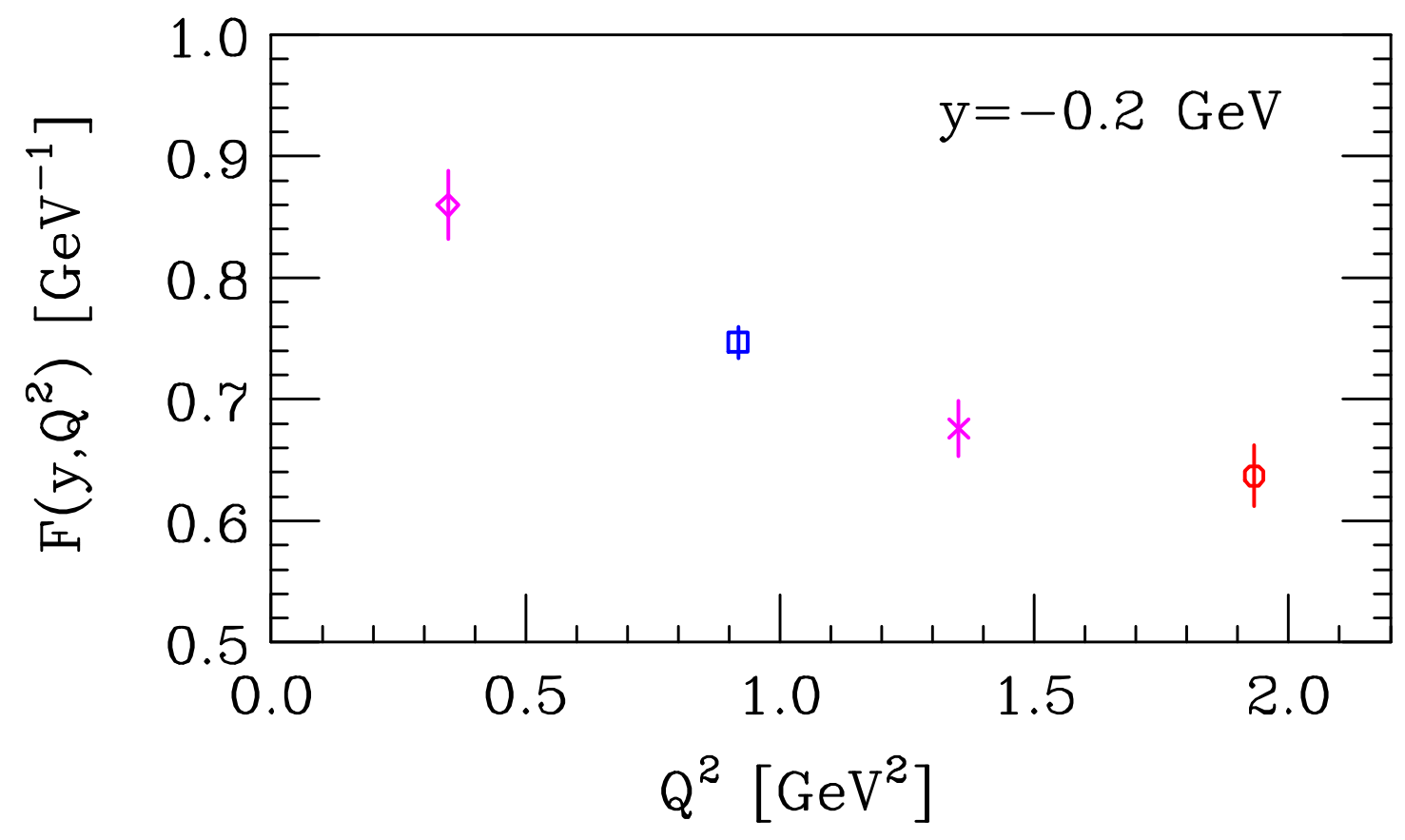

Figure 5.9: $Q^{2}$-dependence of the scaling functions $F\left(y, Q^{2}\right)$ obtained from the cross section displayed in Fig. 5.4 and from the data reported in Ref. [76]. The meaning of the symbols is the same as in Fig. 5.8.

obtained from the data of Day et al [76] using a ${ }_{13}^{27} \mathrm{Al}$ target. The cross sections of Ref. [76] have been measured at fixed beam energy $E=3.595 \mathrm{GeV}$ and scattering angle 16, 20 and $25 \mathrm{deg}$, with the values of $Q^{2}$ corresponding to quasi elastic kinematics being $0.87,1.27$ and $1.78 \mathrm{GeV}^{2}$, respectively.

Scaling behavior is clearly observed at $y \approx 0$, corresponding to region of the quasifree peak, or $\omega \approx Q^{2} / 2 m$. The sizeable scaling violations occurring at larger negative values of $y$ are mainly to be ascribed to the effects of FSI between the knocked-out nucleon and the spectator particles. This feature is illustrated in Fig. 5.9, showing the $Q^{2}$-dependence of the scaling function $F\left(y, Q^{2}\right)$ at fixed $y=-0.2 \mathrm{GeV}$. The approach to the scaling limit from above is a clear signature of FSI. 
Chapter 5. Measurement of the Cross Sections For inclusive electron SCATTERING IN THE E12-14-012 EXPERIMENT AT JEFFERSON LAB

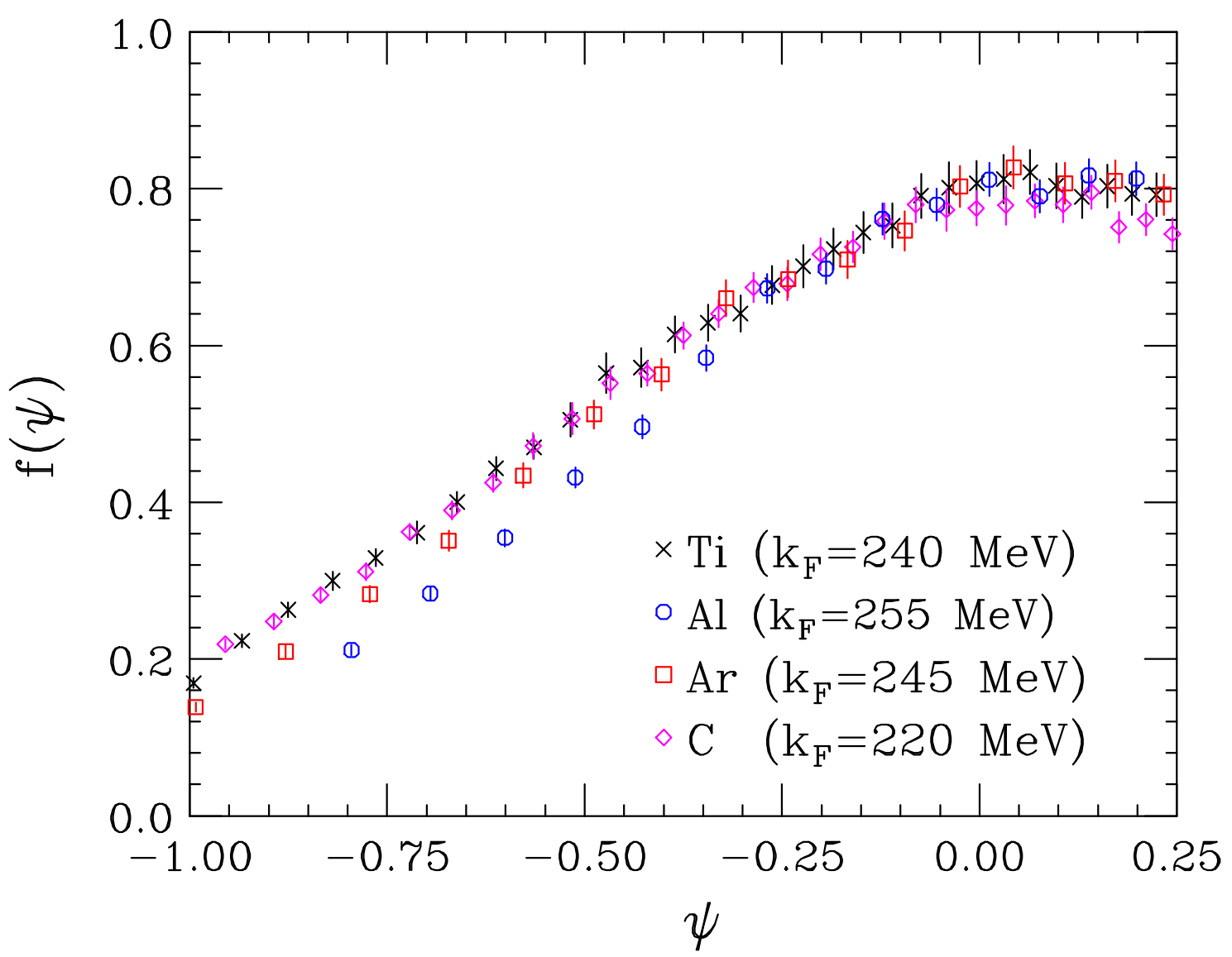

Figure 5.10: Scaling functions of second kind, obtained from the inclusive cross sections measured by the E12-14-012 experiment using carbon, aluminum, argon and titanium targets.

A more general form of scaling, dubbed scaling of second kind, permits a global analysis, combining data corresponding to different targets [83]. The definitions of the dimensionless scaling variable, $\psi$, and scaling function, $f(\psi)$, involve a momentum scale, loosely referred to as nuclear Fermi momentum, providing a parametrization of the target-mass dependence of the measured cross sections.

Figure 5.10 illustrates the scaling functions obtained from the inclusive cross sections measured by the E12-14-012 experiment using carbon, aluminum, argon and titanium targets. The results shows that setting the carbon Fermi momentum to the value obtained from the 
independent analysis of Moniz et al. [84], $k_{F}=220 \mathrm{MeV}$, scaling of the second kind is shown when $k_{F}$ values of 255,245 , and $240 \mathrm{MeV}$ are taken for $\mathrm{Al}$, Ar, and Ti respectively.

A different approach to describe the $A$-dependence of the nuclear inclusive cross section, inspired by the considerations underlying the local density approximation of the nucleus [105], has been proposed by the authors of Ref. [106]. Their analysis - aimed at obtaining the inclusive cross section per nucleon of isospin-symmetric nuclear matter from an extrapolation of the available data - shows that the ${ }^{12} \mathrm{C},{ }^{27} \mathrm{Al},{ }^{56} \mathrm{Fe}$, and ${ }^{197} \mathrm{Au}$ cross sections at $Q^{2}$ ranging between 0.25 and $2.70 \mathrm{GeV}^{2}$ exhibit a striking linear behavior when plotted as a function of $A^{-1 / 3}$. The extension of this study to the data set collected by the E12-14-012 experiment is under way, and the results will be discussed elsewhere.

\subsection{Summary and Conclusions}

We have reported on the measurements of the cross sections for inclusive electron scattering over a broad range of energy transfers, extending from the particle-emission threshold to above the excitation of the first hadronic resonance. These high precision data were taken at Jefferson Lab in Hall A for a beam energy of $E=2.222 \mathrm{GeV}$ and electron scattering angle $\theta=15.54^{\circ}$ from four nuclear targets: carbon, aluminum, argon, and titanium. The reported results give unique opportunity to validate nuclear models employed in Monte Carlo simulations of precise long-baseline neutrino-oscillation experiments, and to assess their contribution to uncertainties of the oscillation analysis in a rigorous manner.

We find (see Fig. 5.6) that the per-nucleon responses for the considered four targets are strikingly similar over the entire energy transfer range $(0.05<\omega<0.90 \mathrm{GeV})$, save at the maximum of the quasielastic peak and the dip region. At the kinematics from the maximum of the quasielastic peak to the onset of the $\Delta$ resonance, the result for carbon stands apart 
from those for aluminum, argon, and titanium. This finding shows that the momentum and energy distribution of nucleons in the nuclear ground state and final-state interactionsinducing the 'Doppler' broadening of the scattered electron's final energy - in carbon is not as pronounced as for the heavier nuclei. When accounting is made for the number of protons and neutrons in each nucleus, this feature does not disappear, as can be seen in Fig. 5.7.

When the aluminum data set along with higher $Q^{2}$ data from SLAC are presented in terms of the $y$-scaling analysis (Fig. 5.8) the set behaves as expected, and the scaling behavior is clearly observed at the kinematics corresponding to the quasi-free peak. While in the absence of FSI, the scaling function $F(y)$ is expected to converge from below with increasing $Q^{2}$, the effect of FSI-falling with $Q^{2}$-leads it to converge from above. These new data fit this pattern (Fig. 5.9).

Taken together this data set will allow us to predict the electromagnetic nuclear responses for nuclei between $A=12$ and 48 by interpolation as a function of $A^{-1 / 3}$. Of particular interest will be oxygen, as water serves as the target and radiator in the large Čerenkov detector of T2K [99], and chlorine, as polyvinyl chloride composes the detectors of NOvA [107]. 


\section{Chapter 6}

\section{Measurement of the $\operatorname{Ar}\left(e, e^{\prime} p\right) X$ and $\operatorname{Ti}\left(e, e^{\prime} p\right) X$ cross sections in Jefferson Lab Hall A}

The work described in this chapter was contributed by: Linjie Gu, Daniel Abrams et al, (The Jefferson Lab Hall A Collaboration). It has been submitted to Physical Review C.

I contributed part of the data analysis work in this paper under Professor Camillo Mariani's supervision.

\subsection{Introduction}

Jefferson Lab experiment E12-14-012 was primarily aimed at obtaining the proton spectral function (SF) of the nucleus ${ }^{40} \mathrm{Ar}$ from a measurement of the cross section of the $\left(e, e^{\prime} p\right)$ reaction

$$
e+A \rightarrow e^{\prime}+p+(A-1)^{*}
$$

in which the scattered electron and the knocked out proton are detected in coincidence. Here $A$ denotes the target nucleus in its ground state, while the recoiling $(A-1)$-nucleon system 
Chapter 6. Measurement of the $\operatorname{Ar}\left(e, e^{\prime} p\right) X$ and Ti $\left(e, e^{\prime} p\right) X$ Cross sections in 104

JefFERSON LAB HaLl A

can be either in the ground state or in any excited state.

Nucleon knockout processes have long been recognized as being ideally suited to study the momentum and removal energy distribution of protons bound in atomic nuclei [108]. Compared to the pioneering studies carried out using proton beams, see, e.g., Ref. [109], $\left(e, e^{\prime} p\right)$ experiments have clear advantages, because they are largely unaffected by strong initial and final state interactions (FSI) between the beam particle and the target, and give access to the properties of deeply bound protons in medium-mass and heavy nuclei [110].

Under the basic assumption that the scattering process involves individual nucleons, and neglecting FSI between the outgoing proton and the spectator nucleons, the momentum and removal energy of the knocked out particle, $\mathbf{p}$ and $E$, can be reconstructed from measured kinematical variables, and the cross section of the process is written in simple factorized form in terms of the spectral function of the target nucleus, $P(\mathbf{p}, E)$, trivially related to the nucleon Green's function, $G(\mathbf{p}, E)$, through

$$
P(\mathbf{p}, E)=\frac{i}{\pi} \operatorname{Im} G(\mathbf{p}, E) .
$$

As a consequence, the spectral function-yielding the probability to remove a proton with momentum $\mathbf{p}$ from the target nucleus leaving the residual system with excitation energy $E-E_{\text {thr }}$, with $E_{\text {thr }}$ being the proton emission threshold - can be readily obtained from the data.

Significant corrections to the somewhat oversimplified scheme outlined above-referred to as Plane Wave Impulse Approximation, or PWIA - arise from the occurrence of FSI. The large body of work devoted to the analysis of $\left(e, e^{\prime} p\right)$ data has provided convincing evidence that the effects of FSI can be accurately included by replacing the plane wave describing the motion of the outgoing proton with a distorted wave, eigenfunction of a phenomenological 
optical potential accounting for its interactions with the mean field of the residual nucleus. In general, the $\left(e, e^{\prime} p\right)$ cross section computed within this approach, known as Distorted Wave Impulse Approximation, or DWIA, involves the off-diagonal spectral function, and cannot be written in factorized form [111]. However, an approximate procedure restoring factorization, referred to as factorized DWIA, has been shown to yield accurate results in the case of parallel kinematics, in which the momentum of the outgoing proton and the momentum transfer are parallel [112]. In this kinematical setup, the spectral function can still be reliably obtained from $\left(e, e^{\prime} p\right)$ data after removing the effects of FSI.

Additional corrections to the PWIA arise from the distortion of the electron wave functions resulting from interactions with the Coulomb field of the nucleus. However, it has been shown that, for nuclei as heavy as ${ }^{40} \mathrm{Ca}$, this effect can be accurately taken into account using an effective momentum transfer [113].

Systematic measurements of $\left(e, e^{\prime} p\right)$ cross sections in the kinematical regime in which the recoiling nucleus is left in a bound state, performed at Saclay [114] and NIKHEF-K [115], have allowed the determination of the spectral functions of a broad set of nuclei. These studies have provided a wealth of information on the energies and momentum distributions of shell-model states belonging to the Fermi sea of the target nuclei, showing at the same time the limitations of the mean-field description and the importance of correlation effects [108].

Besides being a fundamental quantity of nuclear many-body theory, containing important dynamical information, the spectral function is a powerful tool, allowing to obtain the cross sections of a variety of nuclear scattering processes in the kinematical regime in which the beam particles primarily interact with individual nucleons, and FSI can be treated as corrections. Applications to inclusive electron-nucleus scattering have offered vast evidence that the formalism based on spectral functions provides a comprehensive and consistent framework for the calculation of nuclear cross sections in a broad kinematical region, ex- 
Chapter 6. Measurement of the $\operatorname{Ar}\left(e, e^{\prime} p\right) X$ and Ti $\left(e, e^{\prime} p\right) X$ Cross sections in 106

JEFFERSON LAB HALl A

tending from quasielastic (QE) scattering to resonance production and deep-inelastic scattering $[116,117,118]$.

Over the past several years, a great deal of work has been devoted to applying the spectral function formalism to the study of neutrino-nucleus interactions, whose quantitative understanding is needed for the interpretation of accelerator-based searches of neutrino oscillations, see, e.g., Refs. [91, 119]. In this context, it should be noted that the capability to describe a variety of reaction channels within a unified approach is a critical requirement, because the energy of the beam particles is distributed according to a broad flux, typically ranging from a few hundreds of $\mathrm{MeV}$ to a few $\mathrm{GeV}$. Moreover, the knowledge of the spectral function greatly improves the accuracy of reconstruction of the neutrino energy, a key quantity in the oscillation analysis $[121,122]$.

Realistic models of the nuclear spectral functions have been obtained from the approach based on the local density approximation, or LDA, in which the information on the shellmodel structure extracted from $\left(e, e^{\prime} p\right)$ data is combined to the results of accurate calculations of uniform nuclear matter at various densities [117]. The existing calculations of neutrino-nucleus cross sections employing LDA spectral functions [118, 120, 121, 123, 124, $125,126,127,128,129,130,131,132]$, however, are limited to the isospin-symmetric $p$-shell targets ${ }^{16} \mathrm{O}$ and ${ }^{12} \mathrm{C}$. Therefore, the results of these studies are applicable to experiments using water-Čerenkov detectors, e.g. Super-Kamiokande [133], and mineral oil detectors, e.g. MiniBooNE [134].

The analysis of the data collected by the ongoing and future experiments using liquid-argon time-projection chambers, notably the Fermilab Short-Baseline Neutrino program (SBN) [41] and the Deep Underground Neutrino Experiment (DUNE) [135], will require the extension of this approach to the case of a heavier target with large neutron excess. Moreover, in DUNE the proton and neutron spectral functions will both be needed, to extract the Dirac phase 
Table 6.1: Kinematics settings used to collect the data analyzed here.

$\begin{array}{cccccccccc} & \begin{array}{c}E_{e}^{\prime} \\ (\mathrm{MeV})\end{array} & \begin{array}{c}\theta_{e} \\ (\mathrm{deg})\end{array} & \begin{array}{c}Q^{2} \\ \left(\mathrm{GeV}^{2} / c^{2}\right)\end{array} & \begin{array}{c}|\boldsymbol{P}| \\ (\mathrm{MeV} / c)\end{array} & \begin{array}{c}T_{p^{\prime}} \\ (\mathrm{MeV})\end{array} & \begin{array}{c}\theta_{p^{\prime}} \\ (\mathrm{deg})\end{array} & \begin{array}{c}|\mathbf{q}| \\ (\mathrm{MeV} / c)\end{array} & \begin{array}{c}p_{m} \\ (\mathrm{MeV} / c)\end{array} & \begin{array}{c}E_{m} \\ (\mathrm{MeV})\end{array} \\ \mathrm{Ar} & 1,777 & 21.5 & 0.549 & 915 & 372 & -50.0 & 865 & 50 & 73 \\ \mathrm{Ti} & 1,799 & 21.5 & 0.556 & 915 & 372 & -50.0 & 857 & 58 & 51\end{array}$

$\delta_{C P}$ from a comparison of neutrino and antineutrino oscillations, and achieve an accurate description of pion production on protons and neutrons.

In the absence of direct measurements, information on the neutron momentum and removal energy distribution in ${ }_{18}^{40} \mathrm{Ar}$ can be inferred from $\operatorname{Ti}\left(e, e^{\prime} p\right)$ data, exploiting the correspondence between the proton spectrum of titanium, having charge $Z=22$, and the neutron spectrum of argon, having $A-Z=22$. The viability of this procedure is supported by the results of Ref. [136], whose authors have performed a calculation of the inclusive ${ }^{40} \operatorname{Ar}\left(e, e^{\prime}\right)$ and ${ }^{48} \operatorname{Ti}\left(e, e^{\prime}\right)$ cross sections within the framework of the self-consistent Green's function approach. The aim of Jlab experiment E12-14-012, is the determination of the proton spectral functions of argon and titanium from the corresponding $\left(e, e^{\prime} p\right)$ cross sections.

In this article, we present the first results of our analysis. In Sec. 6.2 we discuss the kinematic setup, the detectors and their resolutions, and our definitions of signal and backgrounds. In Sec. 6.3 we introduce the missing energy and the missing momentum, which are the fundamental variables of our analysis, and discuss the main elements of the Monte Carlo (MC) simulations employed for event simulation. Sec. 5.4 is devoted to the uncertainties associated with our analysis, while in Sec. 6.5 the measured missing energy and missing momentum distributions are compared with the MC predictions. Finally, in Sec. 5.7 we summarize our work and draw the conclusions. 
Chapter 6. Measurement of the $\operatorname{Ar}\left(e, e^{\prime} p\right) X$ and Ti $\left(e, e^{\prime} p\right) X$ Cross sections in 108

JefFERSON LAB HaLl A

\subsection{Experimental Setup}

The experiment E12-14-012 was performed at Jefferson lab in Spring 2017. Inclusive $\left(e, e^{\prime}\right)$ and exclusive $\left(e, e^{\prime} p\right)$ electron scattering data were collected on targets of natural argon and natural titanium, as well as on calibration and background targets of carbon and aluminum. The average neutron numbers calculated according to the natural abundances of isotopes are 21.98 for argon and 25.92 for titanium [138]. Therefore, from now on we will refer to the targets considered here as ${ }^{40} \mathrm{Ar}$ and ${ }^{48} \mathrm{Ti}$, for brevity.

The E12-14-012 experiment used an electron beam of energy 2,222 MeV provided by the Continuous Electron Beam Accelerator Facility (CEBAF) at Jefferson Lab. The average beam current was approximately $15 \mu \mathrm{A}$ for the ${ }^{40} \mathrm{Ar}$ target and $20 \mu \mathrm{A}$ for the ${ }^{48} \mathrm{Ti}$ target. The scattered electrons were momentum analyzed and detected in the left high-resolution spectrometer (HRS) in Hall A and the coincident protons were similarly analyzed in the right HRS. The spectrometers are equipped with two vertical drift chambers (VDCs) providing tracking information [33], two scintillator planes for timing measurements and triggering, double-layered lead-glass calorimeter, a gas Čerenkov counter used for particle identification [15], pre-shower and shower detectors (proton arm only) [15] and pion rejectors (electron arm only) [15]. The HRSs were positioned with the electron arm at central scattering angle $\theta_{e}=21.5 \mathrm{deg}$ and the proton arm at an angle $\theta_{p^{\prime}}=-50 \mathrm{deg}$. The beam current and position, the latter being critical for the electron-vertex reconstruction and momentum calculation, were monitored by resonant radio-frequency cavities (beam current monitors, or BCMs [15]) and cavities with four antennae (beam position monitors, or BPMs [15]), respectively. The beam size was monitored using harp scanners, which consists of a thin wire which moves through the beam. We used a raster of $2 \times 2 \mathrm{~mm}^{2}$ area to spread the beam and avoid overheating the target. 
The experiment employed also an aluminum target and a set of carbon targets, used to evaluate backgrounds and monitor the spectrometers optics. The aluminum target was made of two identical foils of the Al-7075 alloy with a thickness of $0.889 \pm 0.002 \mathrm{~g} / \mathrm{cm}^{2}$. One of the aluminum foils was positioned to match the entrance and the other to match the exit windows of the argon gas target cell. The two thick foils were separated by a distance of $25 \mathrm{~cm}$, corresponding to the length of the argon gas cell and the Al foil's thickness.

The analysis presented here uses data collected with the settings given in Table 6.1. All of our data were taken in parallel kinematics, in which the momentum transfer, $\boldsymbol{q}$, and the momentum of the outgoing proton, $\boldsymbol{P}$, are parallel. The only difference of data collection setting for ${ }^{40} \mathrm{Ar}$ and ${ }^{48} \mathrm{Ti}$ is the scattered electron energy.

The VDCs' tracking information was used to determine the momentum and to reconstruct the direction (in-plane and out-of-plane angles) of the scattered electron and proton, and to reconstruct the interaction vertex at the target. We used both the electron and proton arm information separately to reconstruct the interaction vertex and found them in very good agreement. The transformation between focal plane and target quantities was computed using an optical matrix, the accuracy of which was verified using the carbon multi-foil target data and sieve measurements as described in previous papers [101, 137, 138]. Possible variations of the optics and magnetic field in both HRSs are included in the analysis as systematic uncertainties related to the optics.

Several different components were used to build the triggers: the scintillator planes on both the electron and proton spectrometers, along with signals from the gas Čerenkov (GC) detector, the pion rejector (PR), the pre-shower and the shower detector (PS). Table 6.2 lists the trigger configurations, including details on how the signals from the various detector components are combined to form a trigger. 
Chapter 6. Measurement of the $\operatorname{Ar}\left(e, e^{\prime} p\right) X$ and Ti $\left(e, e^{\prime} p\right) X$ Cross sections in 110

JEFFERSON LAB HALl A

Table 6.2: Trigger lists detailing how the signals from different detector components are combined. LEFT and RIGHT identify the electron and proton arm, respectively.

$\begin{array}{ll}\text { T1 } & \left(S_{0} \& \& S_{2}\right) \text { AND }(\mathrm{GC} \| \mathrm{PR})[\mathrm{LEFT}] \\ & \text { AND }(\mathrm{S} 0 \& \& \mathrm{~S} 2)[\mathrm{RIGHT}] \\ \mathrm{T} 2 & \left(S_{0} \| S_{2}\right) \text { AND }(\mathrm{GC} \| \mathrm{PR})[\mathrm{LEFT}] \\ & \text { AND }\left(S_{0} \| S_{2}\right) \text { AND NOT(PS) }[\mathrm{RIGHT}] \\ \mathrm{T} 3 & \left(S_{0} \& \& S_{2}\right) \text { AND }(\mathrm{GC} \| \mathrm{PR})[\mathrm{LEFT}] \\ \mathrm{T} 4 & \left(S_{0} \& \& S_{2}\right)[\mathrm{RIGHT}] \\ \mathrm{T} 5 & \left(S_{0} \| S_{2}\right) \text { AND }(\mathrm{GC}|| \mathrm{PR})[\mathrm{LEFT}] \\ \mathrm{T} 6 & \left(S_{0} \| S_{2}\right) \text { AND NOT}(\mathrm{PS})[\mathrm{RIGHT}]\end{array}$

The triggers used for identifying electron and proton coincidence events were T1 and T2, where $\mathrm{T} 2$ was used to provide a data sample to calculate the overall $\mathrm{T} 1$ trigger efficiency and we were able to compute the efficiency of T1 using also the product of T3 and T4 efficiencies. If the proton and electron observations from the same event were perfectly paired, these values would be the same as $\mathrm{T} 1$ trigger efficiency.

Electrons and protons were selected in their corresponding HRS requiring only one reconstructed good track. For the electron we required also an energy deposit of at least $30 \%$ in the lead calorimeter $\left(E_{\mathrm{cal}} / p>0.3\right)$ and a signal in the Čerekov detector of more than 400 analog-digital-converter (ADC) counts. Furthermore, the tracks were required to be within $\pm 3 \mathrm{mrad}$ of the in-plane angle and $\pm 6 \mathrm{mrad}$ of the out-of-plane angle with respect to the center ray of the spectrometer and have a $d p / p$ of \pm 0.06 . Those latter conditions focused on removing events coming from the acceptance edges of the spectrometers. We used a cut on $\beta$ for the proton arm between 0.6 and 0.8 to further isolate protons. We only included in our analysis events in which both the electron and the proton were recorded in a T1 trigger timing window and for which the difference in the start time of the individual triggers was of just few ns (time coincidence cut). For the argon target we also required that the events originated within the central $\pm 10 \mathrm{~cm}$ of the target cell to exclude contamination from the target entry and exit windows. By measuring events from the thick $\mathrm{Al}$ foils, positioned at 
the same entry and exit window of the target, we determined that the target cell contributions to the measured cross section was negligible $(<0.1 \%)$. The same gas cell was used in another set of experiments and the contribution from an empty gas cell was measured and confirmed a very low contamination of events coming from the Al windows [21]. The spectrometer optics were calibrated using sieve slit measurements and their positions and angles were surveyed before and after moving the spectrometers for each kinematic settings. The survey precision was $0.01 \mathrm{rad}$ and $0.01 \mathrm{~mm}$ respectively for the angle and positions of the spectrometers.

The efficiencies of the elements in the detector stack were studied by comparing rates in various combinations of secondary triggers as in Ref. [101, 137, 138]. Table 6.3 summarizes the efficiency for the trigger, acceptances and kinematical cuts. The live-time of the electronics was computed using the rates from scalers, which were independent of triggered events. The acceptance cuts efficiencies were computed using the MC simulation [68]. The efficiency calculations that are based on MC were evaluated multiple times using slightly different SF models in the MC. The effect of theory models was found to be negligible. Our MC model contains nuclear transparency correction [68, 141], but does not account for all FSI effects. We have studied the role of FSI by looking at kinematical distributions for various MC samples obtained using different ranges of the missing momentum $p_{m}$, defined as in Eq. (6.3), from lower to higher. We found that the electron arm $d p / p$ distributions showed slight variations. We then decided not to use the electron arm $d p / p$ as a kinematical cut in our analysis. The trigger efficiencies were computed using the other available trigger as described above. The time coincidence cut efficiency was evaluated selecting a sample of more pure signal events (using a tighter $\beta$ cut) and looking at the ratio of events with and without the time coincidence cuts. The overall efficiency (between $39.6 \%$ and $48.9 \%$ across all kinematic regions for the ${ }^{40} \mathrm{Ar}$ target, and between $46.8 \%$ and $48.1 \%$ for the ${ }^{48} \mathrm{Ti}$ tar- 
Chapter 6. Measurement of the $\operatorname{Ar}\left(e, e^{\prime} p\right) X$ and Ti $\left(e, e^{\prime} p\right) X$ Cross sections in 112

JEFFERSON LAB HALl A

Table 6.3: Summary of the efficiency analysis for the argon and titanium targets.

\begin{tabular}{lcc} 
& Ar target & Ti target \\
\hline a. Live time & $98.0 \%$ & $98.9 \%$ \\
b. Tracking & $98.3 \%$ & $98.3 \%$ \\
c. Trigger & $92.3 \%$ & $96.9 \%$ \\
d. Cerenkov cut & $99.9 \%$ & $96.6 \%$ \\
e. Calorimeter cut & $97.8 \%$ & $98.1 \%$ \\
f. $\beta$ cut & $95.6 \%$ & $95.3 \%$ \\
g. Coincidence time cut & $54.8 \%$ & $55.5 \%$
\end{tabular}

get) includes cuts on the coincidence triggers, calorimeters, both the lead and the Čerenkov counter, track reconstruction efficiency, live-time, tracking and $\beta$ cut.

\subsection{Data Analysis}

\subsubsection{The $\left(e, e^{\prime} p\right)$ cross section}

In electron-nucleus scattering an incident electron, with energy $E_{e}$, is scattered from a nucleus of mass $M_{A}$ at rest. Electron scattering is generally described in the one-photon exchange approximation, according to which the incident electron exchanges a space-like photon, of energy $\omega$ and momentum $\boldsymbol{q}$, with the target nucleus.

In $\left(e, e^{\prime} p\right)$ experiments the scattered electron and a proton are detected in coincidence in the final state, and their momentum and energy are completely determined. If, in addition, the kinematics is chosen such that the residual nucleus is left in a specific bound state, the reaction is said to be exclusive.

In the following, $\boldsymbol{P}, T_{p^{\prime}}$, and $M$ will denote the momentum, kinetic energy, and mass of the outgoing proton, while the corresponding quantities associated with the recoiling residual nucleus will be denoted $\boldsymbol{p}_{R}, T_{R}$, and $M_{R}$. The missing momentum and missing energy are 
obtained from the measured kinematical quantities using the definitions

$$
\boldsymbol{p}_{m}=\boldsymbol{q}-\boldsymbol{P}=\boldsymbol{p}_{R},
$$

and

$$
E_{m}=\omega-T_{p^{\prime}}-T_{R}
$$

Exploiting energy conservation, implying

$$
\omega+M_{A}=M+T_{p^{\prime}}+M_{R}+T_{R},
$$

and writing the mass of the residual nucleus in the form

$$
M_{R}=M_{A}-M+E_{\mathrm{thr}}+E_{x}=M_{A-1}+E_{x},
$$

where $E_{\text {thr }}$ and $M_{A-1}$ denote the proton emission threshold and the mass of $(A-1)$-nucleon system in its ground state, respectively, Eq. (6.4) can be rewritten

$$
E_{m}=E_{\mathrm{thr}}+E_{x} .
$$

The usual description of the exclusive $\left(e, e^{\prime} p\right)$ reaction in the $\mathrm{QE}$ region assumes the direct knockout mechanism, which naturally emerges within the impulse approximation (IA). According to this picture, the electromagnetic probe interacts through a one-body current with the quasi-free knocked out proton, while all other nucleons in the target act as spectators. In addition, if FSI between the outgoing nucleon and the spectators is negligible, PWIA can 
Chapter 6. Measurement of the $\operatorname{Ar}\left(e, e^{\prime} p\right) X$ and Ti $\left(e, e^{\prime} p\right) X$ Cross sections in 114

JefFERSON LAB HaLl A

be applied, and the $\left(e, e^{\prime} p\right)$ cross section reduces to the factorized form

$$
\frac{d^{6} \sigma}{d \omega d \Omega_{e^{\prime}} d T_{p^{\prime}} d \Omega_{p^{\prime}}}=K \sigma_{e p} P\left(-\boldsymbol{p}_{m}, E_{m}\right)
$$

where $K=|\boldsymbol{P}| E_{p^{\prime}}$, with $E_{p^{\prime}}=\sqrt{\boldsymbol{P}^{2}+M^{2}}$. Here, $\sigma_{e p}$ is the differential cross section describing electron scattering off a bound moving proton, stripped of the flux factor and the energy conserving delta-function $[140,142]$, while $P\left(-\boldsymbol{p}_{m}, E_{m}\right)$ is the proton spectral function of Eq. (6.2). Note that Eqs. (6.3) and (6.4) imply that the arguments of the spectral function can be identified with the initial momentum and the removal energy of the struck nucleon, respectively. Therefore, Eq. (6.8) shows that within PWIA the nuclear spectral function, describing the proton momentum and energy distribution of the target nucleus, can be readily extracted from the measured $\left(e, e^{\prime} p\right)$ cross section.

When FSI are taken into account, and the outgoing proton is described by a distorted wave function as prescribed by DWIA, the initial momentum of the struck nucleon is not trivially related to the measured missing momentum, and the cross section can no longer be written as in Eq. (6.8). However, the occurrence of $y$-scaling in inclusive electron-nucleus scattering $[143,144]$ — whose observation in the analysis of the $\operatorname{Ar}\left(e, e^{\prime}\right)$ and $\operatorname{Ti}\left(e, e^{\prime}\right)$ data is discussed in Refs. [101, 137] —indicates that the formalism based on factorization is still largely applicable in the presence of FSI.

In principle, within the approach of Refs. $[145,146,147]$, the bound and scattering states are both derived from an energy dependent non-Hermitian optical-model Hamiltonian. While being fully consistent, however, this treatment involves severe difficulties. In practice, the bound-state proton wave functions are generally obtained from phenomenological approachesalthough a few studies based on realistic microscopic models of the nuclear Hamiltonian have been carried out for light and medium-heavy nuclei [148, 149]—while the scattering states 
are eigenfunctions of phenomenological optical potentials, the parameters of which are determined through a fit to elastic proton-nucleus scattering data.

The PWIA description provides a clear understanding of the mechanism driving the $\left(e, e^{\prime} p\right)$ reaction, and the ensuing factorized expression of the coincidence cross section, Eq. (6.8), is essential to obtain from the data an intrinsic property of the target, such as the spectral function, independent of kinematics. As pointed out above, however, the occurrence of FSI leads to a violation of factorization, and makes the extraction of the spectral function from the measured cross section more complicated [146, 154]. Additional factorization-breaking corrections arise from the distortion of the electron wave functions, resulting from interactions with the Coulomb field of the target [113, 150, 152].

The general conditions to recover a factorized expression of the cross section are discussed in Refs. $[112,145,146,155,156]$. If these requirements are fulfilled, the DWIA cross section can be written in terms of a distorted spectral function according to

$$
\frac{d^{6} \sigma}{d \omega d \Omega_{e^{\prime}} d T_{p^{\prime}} d \Omega_{p^{\prime}}}=K \sigma_{e p} P^{D}\left(\boldsymbol{P},-\boldsymbol{p}_{m}, E_{m}\right)
$$

Note, however that, unlike the spectral function appearing in Eq. (6.8), the distorted spectral function is not an intrinsic property of the target, because it depends explicitly on the momentum of the outgoing nucleon, which in turn depends on the momentum transfer. The most prominent effects of the inclusion of FSI within the framework of DWIA are a shift and a suppression of the missing momentum distributions, produced by the real and imaginary part of the optical potential, respectively. 
Chapter 6. Measurement of the $\operatorname{Ar}\left(e, e^{\prime} p\right) X$ and Ti $\left(e, e^{\prime} p\right) X$ Cross sections in 116

JefFERSON LAB HaLl A

\subsubsection{Data analysis details}

The measured cross sections are usually analyzed in terms of missing-energy and missingmomentum distributions. For a value of $E_{m}$ corresponding to a peak in the experimental missing-energy distribution, the data are usually presented in terms of the reduced cross section as a function of $p_{m}=\left|\boldsymbol{p}_{m}\right|$. The reduced cross section, obtained from the measured cross section dividing out the kinematic factor $K$ and the electron-proton cross section $\sigma_{e p}$ can be identified with the spectral function in PWIA and with the distorted spectral function in the factorized DWIA of Eq. (6.9). The off-shell extrapolation of de Forest [140, 142] is generally used to describe the bound nucleon cross section.

The experimental reduced cross sections can be compared with the corresponding reduced cross section calculated using different theoretical models. The comparison of the results obtained from the un-factorized and factorized approaches allows one to make an estimate of the accuracy of the factorization scheme, as well as the sensitivity to the different factorizationbreaking contributions.

The six-fold differential cross section as a function of $p_{m}$ and $E_{m}$ was extracted from the data using the $\left(e, e^{\prime} p\right)$ event yield $Y$ for each $p_{m}$ and $E_{m}$ bin

$$
\frac{d^{6} \sigma}{d \omega d \Omega_{e^{\prime}} d T_{p^{\prime}} d \Omega_{p^{\prime}}}=\frac{Y\left(p_{m}, E_{m}\right)}{B \times l t \times \rho \times B H \times V_{B} \times C_{\mathrm{rad}}},
$$

where $B$ is the total accumulated beam charge, $l t$ is the live-time of the detector (fraction of time that the detector was able to collect and write data to disk), $\rho$ is the target density (for argon, corrected for the nominal density of gas in the target cell), $\mathrm{BH}$ is the local density change due to the beam heating the gas cell times the gas expansion due to boiling effects (this correction is not included in the case of ${ }^{48} \mathrm{Ti}$ ), $V_{B}$ is the effect of the acceptance and kinematical cuts, and $C_{\text {rad }}$ is the effect of the radiative corrections and bin center migration. 
We used the SIMC spectrometer package [139] to simulate $\left(e, e^{\prime} p\right)$ events corresponding to our particular kinematic settings, including geometric details of the target cell, radiation correction, and Coulomb effects. SIMC also provided the $V_{B}$ and $C_{\text {rad }}$ corrections as in Eq. (6.10). To simulate the distribution of missing energies and momenta of nucleons bound in the argon and titanium nuclei, SIMC was run with a test SF described in detail in the following subsection.

In Table 6.4 we summarize the energies of the shell model states comprising the ground states of ${ }^{40} \mathrm{Ar}$ and ${ }^{48} \mathrm{Ti}$. In our analysis, in case two orbitals overlap in $E_{m}$, we set the energy range for the orbital to be the same, and we assumed the probability of emission of an electron to be the same. Table 6.4 also lists energies derived from previous data sets, as well as the energy used in the calculation of FSI effects according to the model described in Sec. 6.4.1.

Table 6.4: Parametrization of the missing energy distributions of ${ }_{18}^{40} \mathrm{Ar}$ and ${ }_{22}^{48} \mathrm{Ti}$ assumed in this analysis. The central peak position $E_{\alpha}$, its width $\sigma_{\alpha}$, and the lower (upper) bound on the considered energy range, $E_{\text {low }}^{\alpha}\left(E_{\text {high }}^{\alpha}\right)$ are shown for each level $\alpha$. All values are given in units of $\mathrm{MeV}$.

\begin{tabular}{ccccc}
$\alpha$ & $E_{\alpha}$ & $\sigma_{\alpha}$ & $E_{\text {low }}^{\alpha}$ & $E_{\text {high }}^{\alpha}$ \\
& \multicolumn{4}{c}{ argon } \\
\cline { 2 - 5 } $1 d_{3 / 2}$ & 12.53 & 2 & 8 & 14 \\
$2 s_{1 / 2}$ & 12.93 & 2 & 8 & 14 \\
$1 d_{5 / 2}$ & 18.23 & 4 & 14 & 20 \\
$1 p_{1 / 2}$ & 28.0 & 8 & 20 & 45 \\
$1 p_{3 / 2}$ & 33.0 & 8 & 20 & 45 \\
$1 s_{1 / 2}$ & 52.0 & 8 & 45 & 70 \\
& \multicolumn{4}{c}{ titanium } \\
$1 f_{7 / 2}$ & 11.45 & 2 & 8 & 14 \\
$2 s_{1 / 2}$ & 12.21 & 2 & 14 & 30 \\
$1 d_{3 / 2}$ & 12.84 & 2 & 14 & 30 \\
$1 d_{5 / 2}$ & 15.46 & 4 & 14 & 30 \\
$1 p_{1 / 2}$ & 35.0 & 8 & 30 & 54 \\
$1 p_{3 / 2}$ & 40.0 & 8 & 30 & 54 \\
$1 s_{1 / 2}$ & 62.0 & 8 & 53 & 80
\end{tabular}

SIMC generates events for a broad phase-space, and propagates the events through a detailed 
Chapter 6. Measurement of the $\operatorname{Ar}\left(e, e^{\prime} p\right) X$ and Ti $\left(e, e^{\prime} p\right) X$ Cross sections in 118

JefFerson LaB Hall A

model of the electron and proton spectrometers to account for acceptances and resolution

effects. Each event is weighted by the $\sigma_{c c 1}$ cross section of de Forest [140] and the SF. The final weighted events do not contain any background. As pointed out above, SIMC does not include FSI corrections other than for the nuclear transparency.

The data yield corrected for the above-mentioned factors is then integrated over $E_{m}$ to get the cross section as function of $p_{m}$. We collected 29.6 (12.5) hours of data on $\mathrm{Ar}(\mathrm{Ti})$, corresponding to $\sim 44 \mathrm{k}(13 \mathrm{k})$ events.

We estimated the background due to accidentals to be $2 \%$ (3\%) for $\mathrm{Ar}(\mathrm{Ti})$, performing analysis for each bin of $E_{m}$ and $p_{m}$. First, we selected events in T1 trigger in anti-coincidence between the electron and proton arms. This region corresponds to 100 times the nominal coincidence time window width $(\sim 2 \mathrm{~ns})$. Then, we re-scaled the total number of events found to the width of the coincidence peak to obtain a correct estimate of the background events. The background-event distributions were then generated and subtracted bin by bin from the $E_{m}$ and $p_{m}$ distributions.

\subsubsection{Test spectral functions}

The spectral function employed to simulate events in SIMC is based on the simplest implementation of the nuclear shell model,

$$
P\left(\boldsymbol{p}_{m}, E_{m}\right)=\sum_{\alpha}\left|\phi_{\alpha}\left(\boldsymbol{p}_{m}\right)\right|^{2} f_{\alpha}\left(E_{m}-E_{\alpha}\right)
$$

where the sum runs over all occupied states. In the above equation, $\phi_{\alpha}\left(\boldsymbol{p}_{m}\right)$ is the momentumspace wave function of the state $\alpha$, normalized to unity, and $f_{\alpha}\left(E_{m}-E_{\alpha}\right)$ represents the distribution of missing energy peaked at the value $E_{\alpha}$, reflecting the width of the corre- 


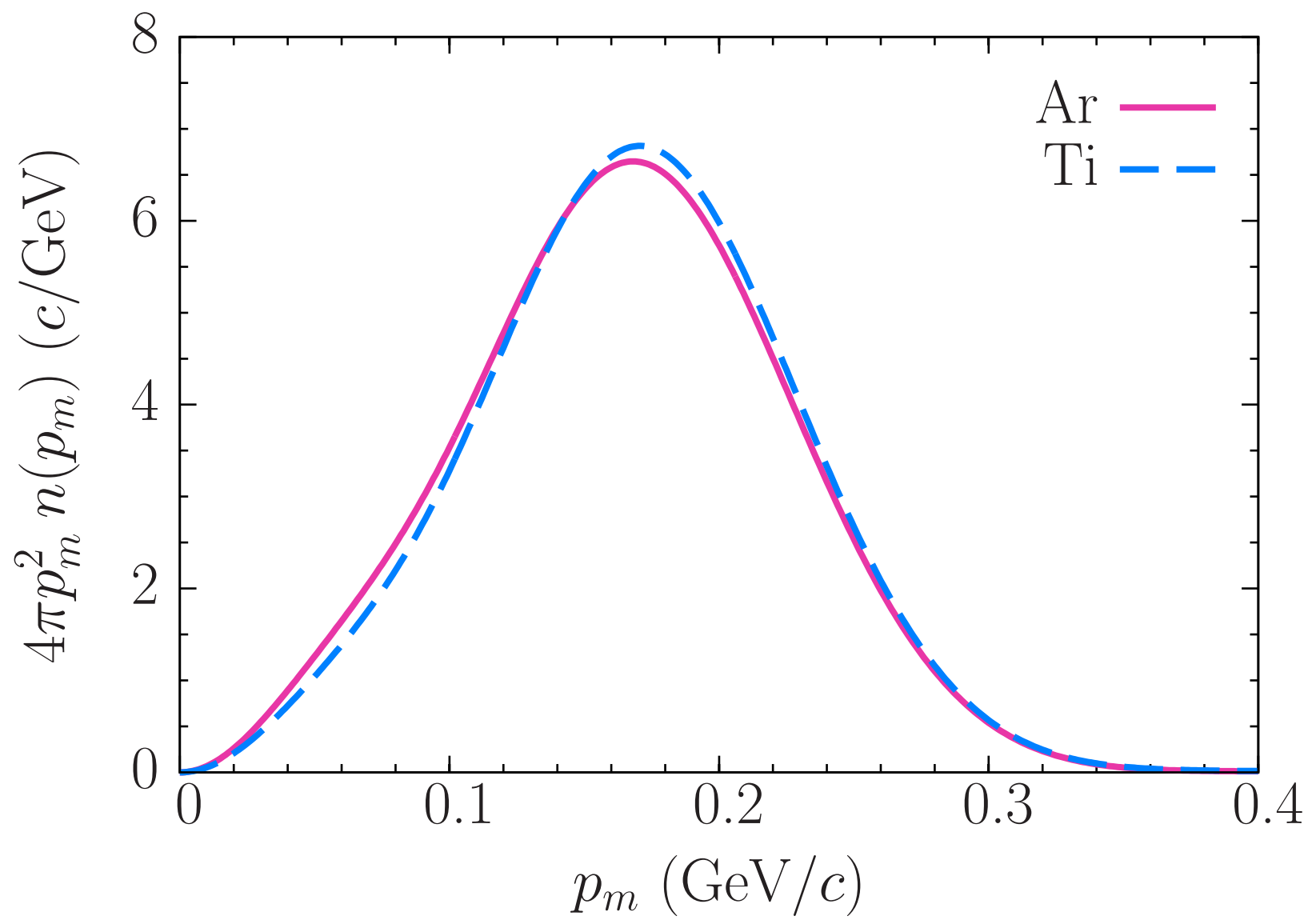

Figure 6.1: Missing momentum distributions of protons in argon and titanium assumed in this analysis.

sponding state. As a consequence of deviations from this mean-field picture originating from nucleon-nucleon correlations, we expect the Monte Carlo simulations typically to overestimate the data, due to the partial depletion of the shell-model states and to the correlated contribution to the nuclear spectral function.

We compared the momentum distribution, defined as

$$
n\left(p_{m}\right)=\int P\left(p_{m}, E_{m}\right) d E_{m}
$$

obtained using the wave functions of Refs. [157, 158] and Ref. [159], and found that the 
Chapter 6. Measurement of the $\operatorname{Ar}\left(e, e^{\prime} p\right) X$ and $\operatorname{Ti}\left(e, e^{\prime} p\right) X$ Cross sections in 120

JEFFERSON LAB HALL A

differences between them are negligible for both argon and titanium. As shown in Fig. 6.1, the momentum distributions for argon and titanium also turn out not to differ significantly. This finding suggests that nuclear effects in argon and titanium are similar.

The missing energy distributions are assumed to be Gaussian

$$
f_{\alpha}\left(E_{m}-E_{\alpha}\right)=\frac{1}{\sqrt{2 \pi} \sigma_{\alpha}} \exp \left[-\frac{\left(E_{m}-E_{\alpha}\right)^{2}}{2 \sigma_{\alpha}^{2}}\right] .
$$

We obtain the missing energies of the least-bound valence orbital for protons - corresponding to the residual nucleus being left in the ground state, with an additional electron and the knocked-out proton at rest - from the mass difference of the residual system and the target nucleus [160]. These values of missing energy, corresponding to the $1 d_{3 / 2}\left(1 f_{7 / 2}\right)$ state for ${ }_{18}^{40} \mathrm{Ar}\left({ }_{22}^{48} \mathrm{Ti}\right)$ in Table 6.4 , are given by

$$
E_{\mathrm{thr}}=M_{A-1}+M+m-M_{A},
$$

where $m$ stands for the electron mass.

In principle, the energies of other valence levels of ${ }_{18}^{40} \mathrm{Ar}$ and ${ }_{22}^{48} \mathrm{Ti}$ could be obtained from the excitation spectra of ${ }_{17}^{39} \mathrm{Cl}[161]$ and ${ }_{21}^{47} \mathrm{Sc}$ [162]. However, the fragmentation of shell-model states induced by long-range correlations makes this information difficult to interpret within the independent-particle model, assumed in Eq. (6.11), because a few spectroscopic lines typically correspond to a given spin-parity state. To overcome this issue and identify the dominant lines, we rely on the spectroscopic strengths determined in past direct pick-up experiments such as $A\left({ }_{1}^{2} \mathrm{H},{ }_{2}^{3} \mathrm{He}\right)$ for argon [163] and titanium [164].

The heavily fragmented $1 d_{5 / 2}$ shell $[163,164]$ — with over 10 , densely packed, spectroscopic 


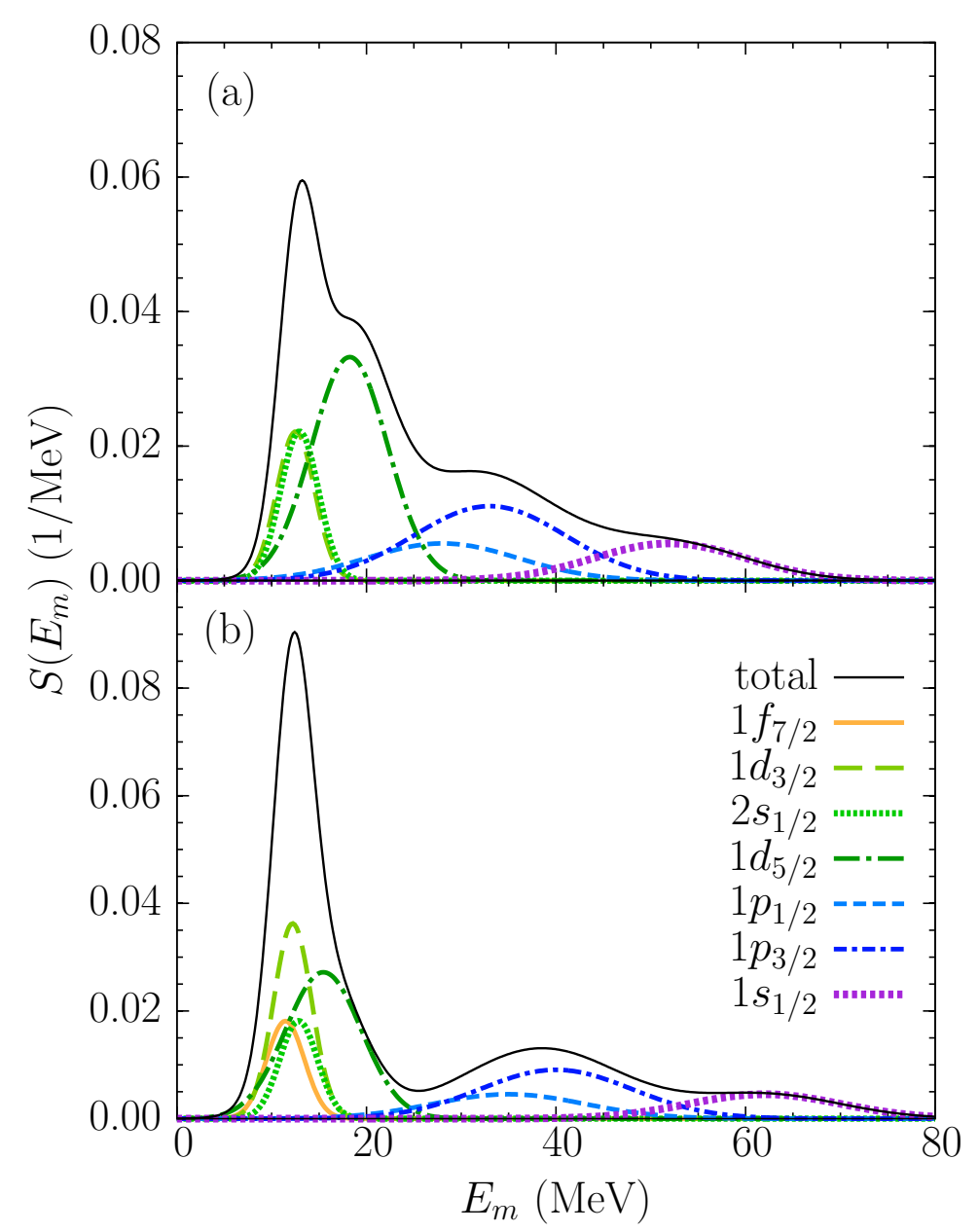

Figure 6.2: Missing energy distribution of protons in (a) argon and (b) titanium assumed in this analysis.

lines contributing - can be expected to lend itself well to the approximation by a single distribution of finite width. To determine its peak position, in addition to the experimental data [163, 164], we use the theoretical analyses of Refs. [165, 166] as guidance.

More deeper-lying shells- $1 p_{1 / 2}, 1 p_{3 / 2}$, and $1 s_{1 / 2}$ - were not probed by the past experiments $[163,164]$. Their $E_{\alpha}$ values, as well as the widths $\sigma_{\alpha}$ for all shells, are determined to provide a reasonable description of the missing-energy distributions obtained in this experiment. The resulting parametrization is detailed in Table 6.4, and presented in Fig. 6.2. 
Chapter 6. Measurement of the $\operatorname{Ar}\left(e, e^{\prime} p\right) X$ and $\operatorname{Ti}\left(e, e^{\prime} p\right) X$ Cross sections in 122

JEFFERSON LAB HALL A

Table 6.5: Contributions to systematical uncertainties for argon and titanium.

\begin{tabular}{lcc} 
& $\mathrm{Ar}$ & $\mathrm{Ti}$ \\
\hline \hline 1. Total statistical uncertainty & $0.53 \%$ & $0.78 \%$ \\
2. Total systematic uncertainty & $2.75 \%$ & $2.39 \%$ \\
a. Beam $x \& y$ offset & $0.56 \%$ & $0.48 \%$ \\
\hline b. Beam energy & $0.10 \%$ & $0.10 \%$ \\
\hline c. Beam charge & $0.30 \%$ & $0.30 \%$ \\
\hline d. HRS $x \& y$ offset & $0.72 \%$ & $0.69 \%$ \\
\hline g. Optics $(\mathrm{q} 1, \mathrm{q} 2, \mathrm{q} 3)$ & $1.10 \%$ & $0.34 \%$ \\
\hline h. Acceptance cut $(\theta, \phi, z)$ & $1.23 \%$ & $1.39 \%$ \\
\hline i. Target thickness/density/length & $0.2 \%$ & $0.2 \%$ \\
\hline j. Calorimeter\&Cerenkov cut & $0.02 \%$ & $0.02 \%$ \\
\hline k. Radiative and Coulomb corr. & $1.00 \%$ & $1.00 \%$ \\
\hline l. $\beta$ cut & $0.63 \%$ & $0.48 \%$ \\
\hline m. Boiling effect & $0.70 \%$ & - \\
\hline n. Cross section model & $1.00 \%$ & $1.00 \%$ \\
\hline o. Trigger and coincidence time cut & $0.99 \%$ & $0.78 \%$ \\
\hline \hline Total systematic uncertainty & $2.75 \%$ & $2.39 \%$ \\
\hline
\end{tabular}

\subsection{Uncertainty Analysis}

The total systematic uncertainty in this analysis was estimated by summing in quadrature the contributions listed in Table 6.5. We determined the kinematic and acceptance cuts ensuring that there are no dependencies on kinematic variables and input theory model, in this way all uncertainties are bin to bin uncorrelated. All the kinematic and acceptance cuts were varied by the resolution of the variable under consideration. The MC used to evaluate those uncertainties did not contain effects due to FSI except for the transparency corrections. A priori the MC simulation could depend on the underlying theoretical model. However, we repeated the analysis of systematic uncertainties varying its ingredients, and did not observe any substantial variations of the obtained results. The effects of FSI, as pointed out above, are a quenching of the strength of the cross section and a modification of the kinematic of the outgoing particles. 


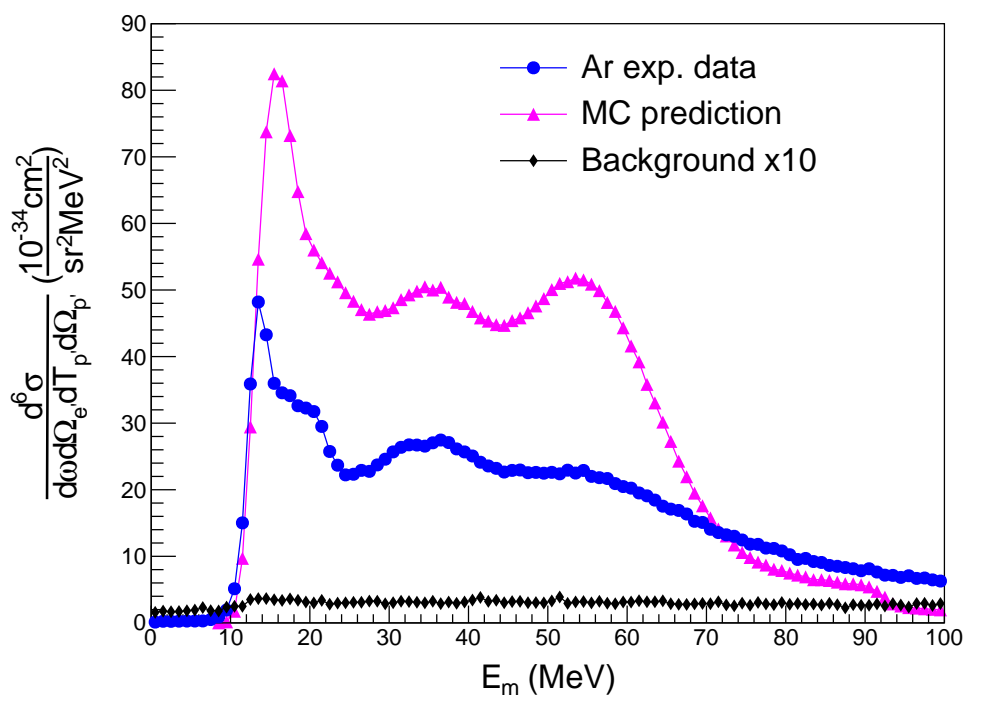

Figure 6.3: Six-fold differential cross section as a function of missing energy for argon. The background estimate (line connecting the experimental data points) is multiplied by 10 for purpose of presentation. The MC predictions, based on the mean-field SF, include a correction for the nuclear transparency, while other FSI effects are not accounted for.

The statistical uncertainties for $\mathrm{Ar}(\mathrm{Ti})$ was determined to be $0.53 \%$ (0.78\%). As the obtained results depend on the Monte Carlo calculation, it is important to estimate uncertainties resulting from its inputs. To determine the uncertainties related to the target position, we performed the simulation with the inputs for the beam's and spectrometer's $x$ and $y$ offsets varied within uncertainties, and we recomputed the optical transport matrix varying the three quadrupole magnetic fields, one at the time. Each of these runs was compared to the reference run, and the corresponding differences were summed in quadrature to give the total systematic uncertainty due to the Monte Carlo simulation.

The uncertainties related to the calorimeter and Čerenkov detectors were determined by changing the corresponding cut by a small amount and calculating the difference with respect to the nominal yield value. The uncertainty due to the acceptance cuts on the angles was calculated using the same method. We included an overall fixed uncertainty for both the beam charge and beam energy, as in the previous work on C, Ti, Ar, and Al [101, 137, 138]. 
Chapter 6. Measurement of the $\operatorname{Ar}\left(e, e^{\prime} p\right) X$ and Ti $\left(e, e^{\prime} p\right) X$ Cross sections in 124

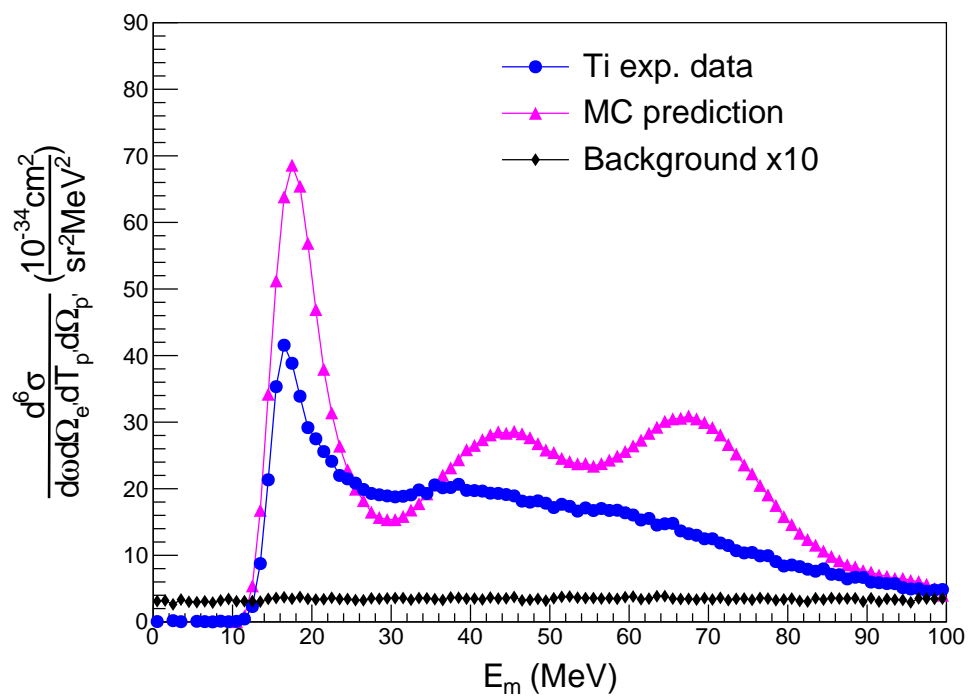

Figure 6.4: Six-fold differential cross section as a function of missing energy for titanium. The background estimate (line connecting the experimental data points) is multiplied by 10 for purpose of presentation. The MC predictions, based on the mean-field SF, include a correction for the nuclear transparency, while other FSI effects are not accounted for.

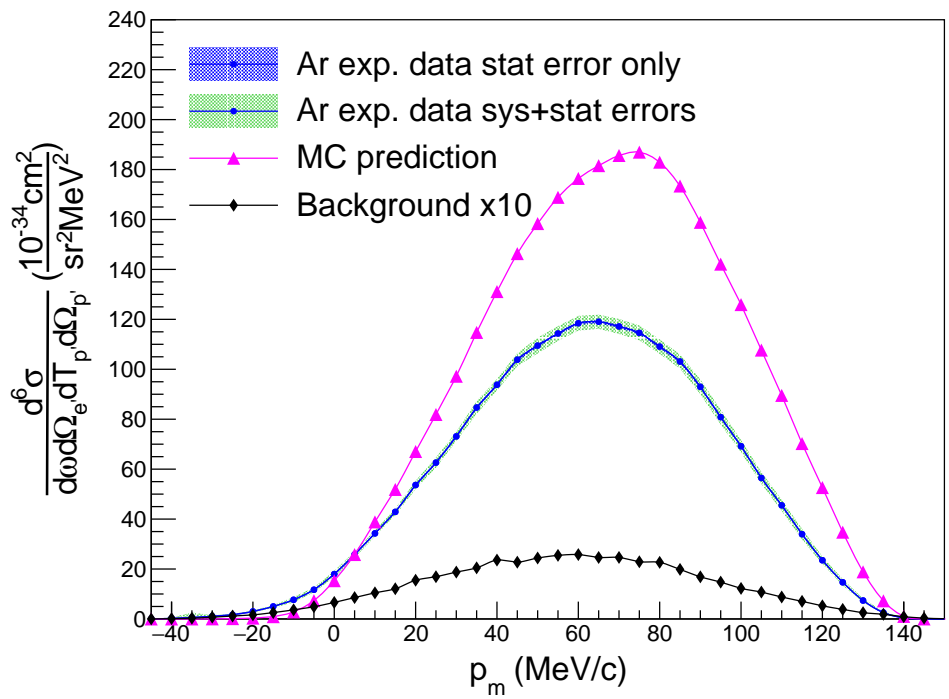

Figure 6.5: Same as Fig. 6.3 but for the cross section as a function of missing momentum. The inner (outer) uncertainty bands correspond to statistical (total) uncertainties.

We evaluated the systematic uncertainties related to the trigger efficiency by determining variations across multiple runs, as well as by applying different acceptance cuts. A fixed uncertainty was assigned to take care of those variations. 


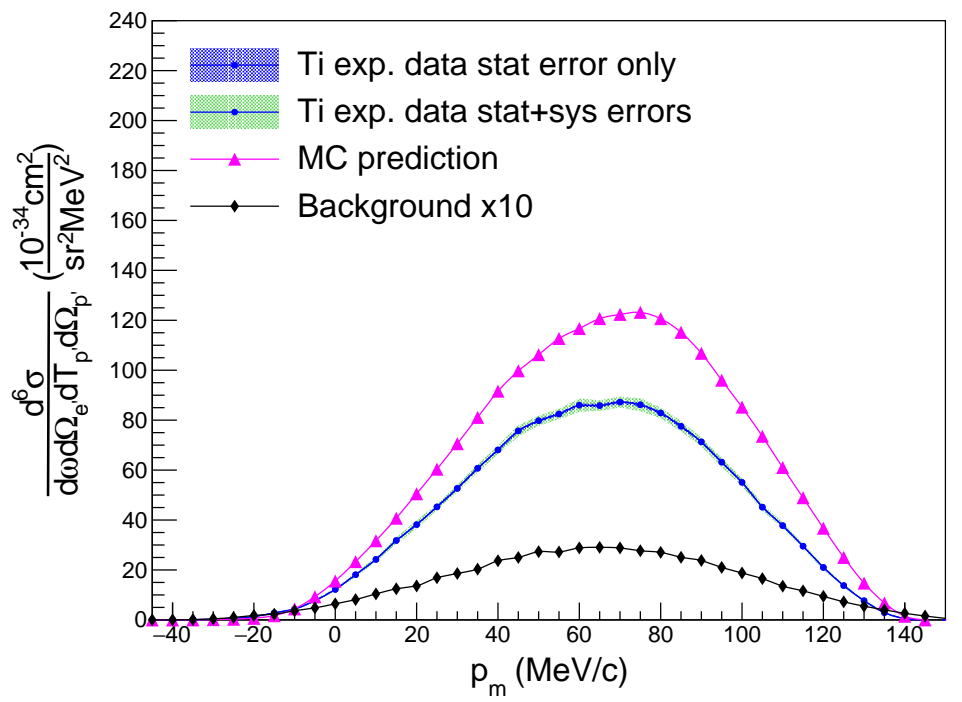

Figure 6.6: Same as Fig. 6.3 but for the cross section as a function of missing momentum. The inner (outer) uncertainty bands correspond to statistical (total) uncertainties.

The time-coincidence cut efficiency, as other acceptance cuts, was evaluated by changing the cut by $\pm \sigma$.

SIMC generates events including the effects from radiative processes: vacuum polarization, vertex corrections, and internal bremsstrahlung. External radiative processes refer to electrons losing energy while passing through material in the target. Radiative correction in SIMC are implemented following the recipe of Dasu [23], using the Whitlow's approach $[22,167]$. We considered a fixed $1 \%$ uncertainty due to the theoretical model for the radiative corrections over the full kinematic range as in our previous work. We generated different $\mathrm{MC}$ where the radiative corrections were re-scaled by $\sqrt{\left(Q^{2}\right)} / 2, Q^{2}$ being the four-momentum transfer squared, and re-analyzed the data and looked for variations. Coulomb corrections were included in the local effective momentum approximation [168]. A $10 \%$ uncertainty associated with the Coulomb potential was included as systematic uncertainty. Finally, we included a target thickness uncertainty and an uncertainty due to the boiling effect correction [21]. 
Chapter 6. Measurement of the $\operatorname{Ar}\left(e, e^{\prime} p\right) X$ and Ti $\left(e, e^{\prime} p\right) X$ Cross sections in 126

JEFFERSON LAB HALl A

The measured and MC predicted differential cross sections $d^{6} \sigma / d \omega d \Omega_{e} d p d \Omega_{p}$ are presented in Fig. 6.3, 6.4 as a function of $E_{m}$ and in Fig. 6.5, 6.6 as a function of $p_{m}$, integrated over the full range of $E_{m}$, for ${ }^{40} \mathrm{Ar}$ and ${ }^{48} \mathrm{Ti}$ targets.

The MC simulation clearly overestimates the extracted cross sections. As the nuclear model underlying the simulation neglects the effects of FSI other than the nuclear transparency and all correlations between nucleons, this difference is by no means surprising. Both FSI and partial depletion of the shell-model states require further studies, base on all five datasets collected by the JLab E12-14-012 experiment, which will be reported elsewhere.

\subsubsection{Final state interactions}

Within DWIA, FSI between the outgoing proton and the spectator nucleons are described by a complex, energy dependent, phenomenological optical potential (OP). The OPs available for calculations were determined by fitting a set of elastic proton-nucleus scattering data for a range of target nuclei and beam energies. Different parametrizations, yielding equivalently good descriptions of the data, can give differences and theoretical uncertainties when "equivalent" OPs are used in kinematical regions for which experimental data are not available, or when they are extended to inelastic scattering and to calculation of the cross section of different nuclear reactions.

Nonrelativistic and relativistic OPs are available for $\left(e, e^{\prime} p\right)$ calculations within nonrelativistic and relativistic DWIA frameworks. However, nonrelativistic phenomenological OPs are available for energies not larger than $200 \mathrm{MeV}$. It is generally believed that above 180 $\mathrm{MeV}$ the Schrödinger picture of the phenomenological OP should be replaced by a Dirac approach, and a relativistic OP should be used. In Ref. [153], it was shown that in $\left(e, e^{\prime} p\right)$ reactions the differences between the nonrelativistic and relativistic DWIA results depend 


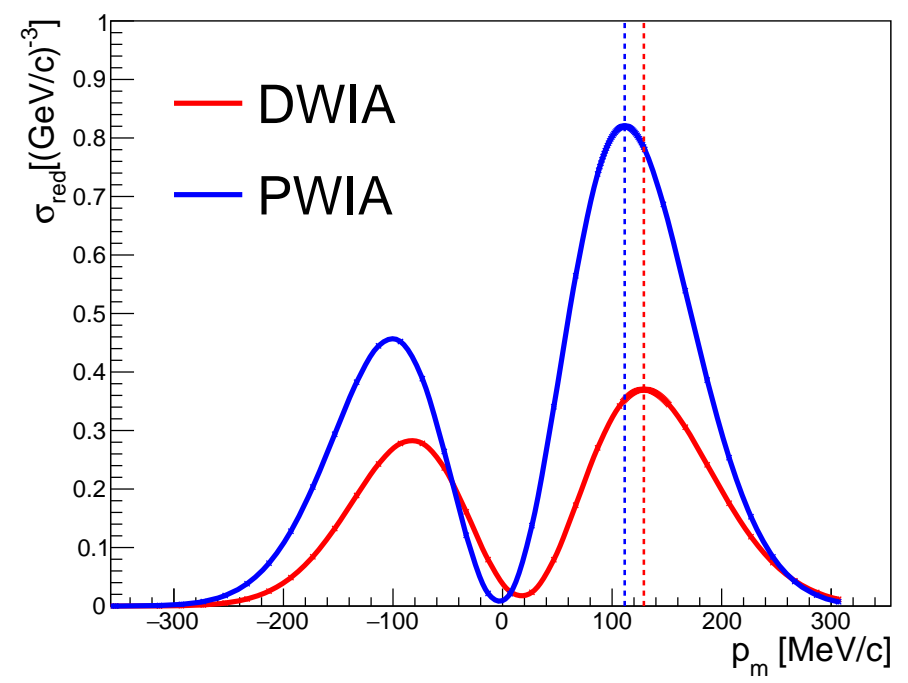

Figure 6.7: Reduced cross section as a function of missing momentum for the $1 p_{1 / 2}$ proton knockout from argon. We compare the PWIA and DWIA results obtained for the parallel kinematics considered in this analysis.

on kinematics and increase with the outgoing proton energy, and for proton energies above $200 \mathrm{MeV}$ a relativistic calculation is necessary.

We have used the so-called "democratic"(DEM) relativistic OP [169], obtained from a global fit to over 200 sets of elastic proton-nucleus scattering data, comprised of a broad range of targets, from helium to lead, at energies up to 1,040 MeV.

An example of the comparison between PWIA and DWIA results is given in Fig. 6.7, where the reduced cross section as a function of $p_{m}$ is displayed for proton knockout from the $1 p_{1 / 2}$ argon orbital. Calculations are performed within the relativistic model of Ref. [153] for the parallel kinematics of the present experiment. Positive and negative values of $p_{m}$ indicate, conventionally, cases in which $|\boldsymbol{q}|<\left|\boldsymbol{p}^{\prime}\right|$ and $|\boldsymbol{q}|>\left|\boldsymbol{p}^{\prime}\right|$, respectively. The reduction and the shift produced in the reduced cross section by FSI in the DWIA calculation can be clearly seen.

The two dashed lines drawn in the region of positive $p_{m}$ of the figure indicate the value of 
Chapter 6. Measurement of the $\operatorname{Ar}\left(e, e^{\prime} p\right) X$ and Ti $\left(e, e^{\prime} p\right) X$ Cross sections in 128

JefFERSON LAB HaLl A

Table 6.6: Shifts between the reduced DWIA and PWIA cross sections, and the DWIA to PWIA cross-section ratios, obtained for proton knockout from various argon orbital using different optical potentials: DEM [169], EDAD3 [170], and EDAD1 [170]. All results are calculated for $p_{m}>0$.

\begin{tabular}{ccccccc} 
& \multicolumn{3}{c}{ Shift $(\mathrm{MeV} / c)$} & \multicolumn{3}{c}{ DWIA/PWIA } \\
\cline { 2 - 7 } Orbital & EDAD1 & EDAD3 & DEM & EDAD1 & EDAD3 & DEM \\
$1 d_{3 / 2}$ & 1.5 & -2.0 & 1.5 & 0.58 & 0.57 & 0.58 \\
$2 s_{1 / 2}$ & 8.0 & 7.0 & 8.0 & 0.78 & 0.78 & 0.78 \\
$1 d_{5 / 2}$ & -2.0 & -6.5 & -3.0 & 0.57 & 0.57 & 0.58 \\
$1 p_{1 / 2}$ & 12.5 & 9.0 & 12.5 & 0.43 & 0.39 & 0.42 \\
$1 p_{3 / 2}$ & 9.5 & 5.0 & 9.0 & 0.47 & 0.44 & 0.46 \\
$1 s_{1 / 2}$ & 13.0 & 10.0 & 13.0 & 0.42 & 0.38 & 0.41
\end{tabular}

$p_{m}$ corresponding to the peaks of the DWIA and PWIA reduced cross sections. We use the distance between the two dashed lines as a measure of the shift produced by FSI.

The reduction of the calculated cross section produced by FSI can be measured by the DWIA/PWIA ratio, which is defined here as the ratio of the integral over $p_{m}$ of the DWIA and PWIA reduced cross sections. Both the shift and the DWIA/PWIA ratios are computed separately for the positive and negative $p_{m}$ regions.

The theoretical uncertainty of the shift and the reduction produced by FSI has been evaluated investigating the sensitivity of the DWIA and PWIA results to different choices of the theoretical ingredients of the calculation.

The uncertainty due to the choice of the OP has been evaluated by comparing the results obtained with the DEM and other energy-dependent and atomic-number dependent relativistic OPs, referred to as EDAD1 and EDAD3 [170]. The shift and the DWIA/PWIA ratio in the positive $p_{m}$ region, computed for proton knock out from various argon orbitals using the DEM, EDAD1, and EDAD3 potentials are reported in Table 6.6. The results indicate a slight dependence of FSI effects on the choice of OP.

Note that the three OPs were determined by a fitting procedure of elastic proton scattering 


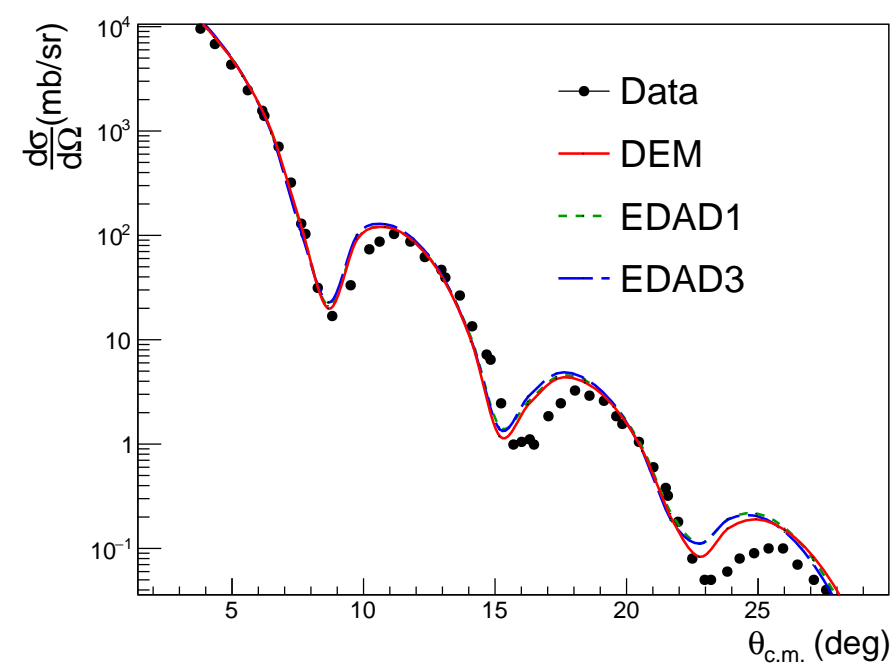

Figure 6.8: Differential cross section for elastic proton scattering on ${ }^{40} \mathrm{Ar}$ at $0.8 \mathrm{GeV}$ as a function of scattering angle. Results for the DEM, EDAD1, and EDAD3 optical potentials, which turn out to almost completely overlap, are compared with the experimental data [171].

data over a wide range of nuclei, which, however, did not include argon. This means that the ability of the phenomenological OPs to describe elastic proton scattering data on argon is not guaranteed. A test of this ability is presented in Fig. 6.8, where the ${ }^{40} \operatorname{Ar}\left(p, p^{\prime}\right)$ cross section calculated at $0.8 \mathrm{GeV}$ with the three OPs is compared to the corresponding experimental cross section obtained using the HRS of the Los Alamos Meson Physics Facility [171]. The results of the three OPs largely overlap, and their agreement with the experimental cross section, although not perfect, is more than reasonable, in particular if we consider that it has not been obtained from a fit to the data.

In the relativistic DWIA and PWIA calculations different current conserving (cc) expressions of the one-body nuclear current operator can be adopted. The different expressions are equivalent for on-shell nucleons, while differences can arise for off-shell nucleons. For all the results that we have presented until now, and as a basis for the present calculations, we have adopted the cc1 prescription [140]. We note that, historically, the cc1 cross section has been often used to obtain the reduced cross section from the experimental and theoretical cross 
Chapter 6. Measurement of the $\operatorname{Ar}\left(e, e^{\prime} p\right) X$ and Ti $\left(e, e^{\prime} p\right) X$ Cross sections in 130

JefFERSON LAB HaLl A

section. The impact of using a different cross section — such as the cc2 model of Ref. [140] — in the determination of the spectral function will be discussed in future analysis.

We have also checked that the differences obtained using different proton form factors in the calculation of the nuclear current are always negligible in the kinematic situation of the present experiment.

The bound proton states adopted in the calculations are self-consistent Dirac-Hartree solutions derived within a relativistic mean field approach using a Lagrangian containing $\sigma, \omega$, and $\rho$ mesons, with medium dependent parametrizations of the meson-nucleon vertices that can be more directly related to the underlying microscopic description of nuclear interactions $[157,158]$. Pairing effects have been included carrying out Bardeen-Cooper-Schrieffer (BCS) calculations. The theoretical uncertainties on the shift and the DWIA/PWIA ratio due to the use of wave functions obtained with a different description of pairing, based on the relativistic Dirac-Hartree-Bogoliubov (DHB) model [159], turn out to be negligible.

In our analysis we assumed the missing energy distribution for each of the orbitals in ${ }^{40} \mathrm{Ar}$ and ${ }^{48} \mathrm{Ti}$ as shown in Fig. 6.2. The lower and upper energy bounds assumed in the DWIA analysis of FSI are given for each orbital in Table 6.4. The FSI correction has been applied event by event in both the missing energy and missing momentum distributions. We applied different corrections for events with $|\boldsymbol{q}|<\left|\boldsymbol{p}^{\prime}\right|$ and $|\boldsymbol{q}|>\left|\boldsymbol{p}^{\prime}\right|$, according to the theoretical predictions mentioned before. For each event, we used the reconstructed energy and momentum of both electron and proton to determine the orbital involved in the primary interaction. Then, we applied the FSI correction, based on the $p_{m}$ sign. For orbitals that overlap we use a simple prescription to determine the most probable orbital from which the electron was emitted, as described in Sec. 6.3.2. 


\subsection{Differential cross section comparison}

Figures $6.9,6.10,6.11,6.12,6.13$ and 6.14 show a comparison between the measured differential cross sections of ${ }^{40} \mathrm{Ar}$ and ${ }^{48} \mathrm{Ti}$ and $\mathrm{MC}$ predictions including full FSI corrections, plotted as a function of $p_{m}$ for three different ranges of $E_{m}$. The missing energy regions for ${ }^{40} \mathrm{Ar}\left({ }^{48} \mathrm{Ti}\right)$ are: $E_{m}<27 \mathrm{MeV}\left(E_{m}<30 \mathrm{MeV}\right), 27<E_{m}<44 \mathrm{MeV}\left(30<E_{m}<54 \mathrm{MeV}\right)$ and $44<E_{m}<70 \mathrm{MeV}\left(54<E_{m}<90 \mathrm{MeV}\right)$.

We estimated the background to be of the order $2 \%$ for ${ }^{40} \mathrm{Ar}$ and $3 \%$ for ${ }^{48} \mathrm{Ti}$. The MC systematic uncertainties from FSI are estimated by varying the following ingredients of the model:

(i) the optical potential (DEM, EDAD1, or EDAD3);

(ii) the pairing mechanism underlying the determination of the wave functions (the default BCS model $[157,158]$ or the DHB model $[159])$;

(iii) the parametrization of the nucleon form factors.

The total systematic uncertainty is obtained by adding in quadrature all the variations, and including an overall uncertainty of the theoretical model of $15 \%$.

A prominent feature of both Figs. 6.9, 6.10, 6.11, 6.12, 6.13 and 6.14 is that the agreement between data and MC predictions including FSI, which turns out to be quite good in the region of low missing energies, becomes significantly worse at larger $E_{m}$. This behavior can be explained considering that, according to the shell-model picture employed in MC simulations, missing energies $E_{m}>27 \mathrm{MeV}$ correspond to proton knockout from the deeply bound $1 p_{1 / 2}, 1 p_{3 / 2}$, and $1 s_{1 / 2}$ states.

As discussed in Sec. 6.3.3, the energies and widths of these states are only estimated, and 
Chapter 6. Measurement of the $\operatorname{Ar}\left(e, e^{\prime} p\right) X$ and Ti $\left(e, e^{\prime} p\right) X$ cross sections in 132

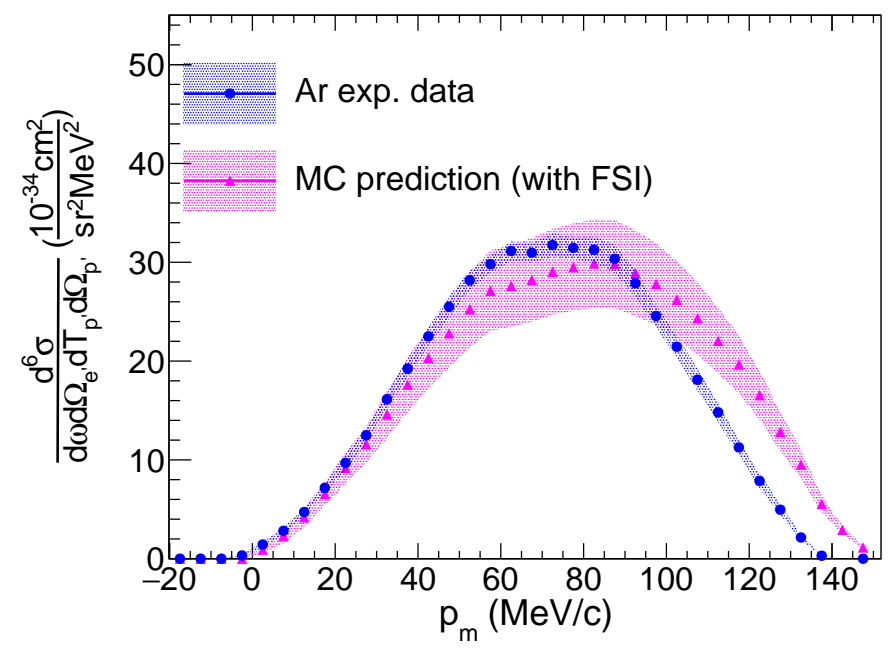

Figure 6.9: Six-fold differential cross section for argon as a function of missing momentum integrated over $0<E_{m}<27 \mathrm{MeV}$. The background estimate is multiplied by 10 for presentation. The MC predictions, based on the mean-field SF, include the full FSI corrections.

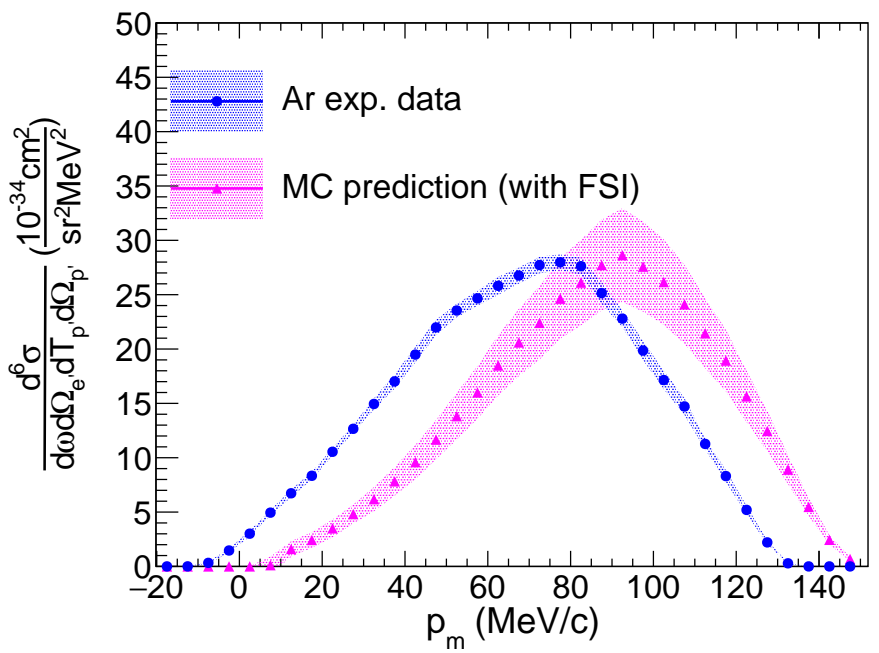

Figure 6.10: Six-fold differential cross section for argon as a function of missing momentum integrated over $27<E_{m}<44 \mathrm{MeV}$. The background estimate is multiplied by 10 for presentation. The MC predictions, based on the mean-field SF, include the full FSI corrections. 


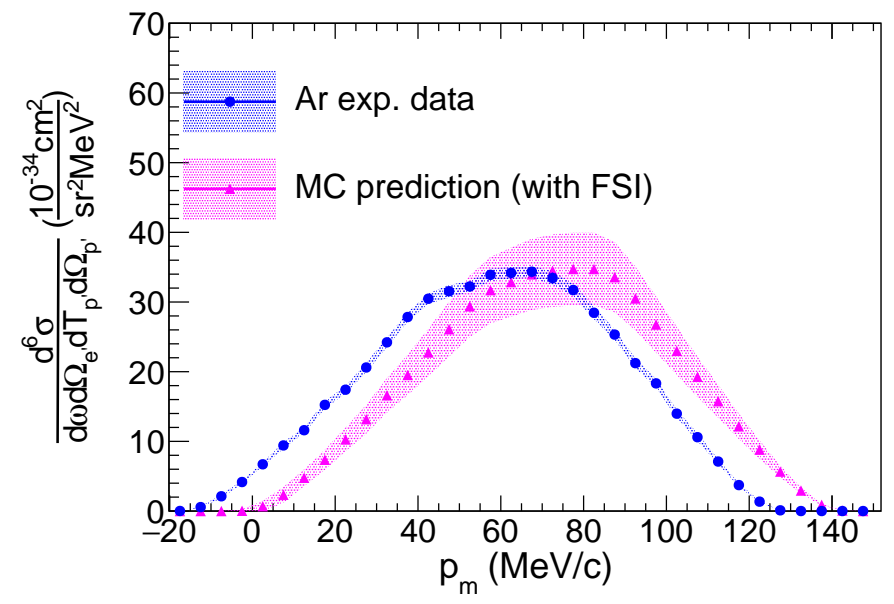

Figure 6.11: Six-fold differential cross section for argon as a function of missing momentum integrated over $44<E_{m}<70 \mathrm{MeV}$. The background estimate is multiplied by 10 for presentation. The MC predictions, based on the mean-field SF, include the full FSI corrections.

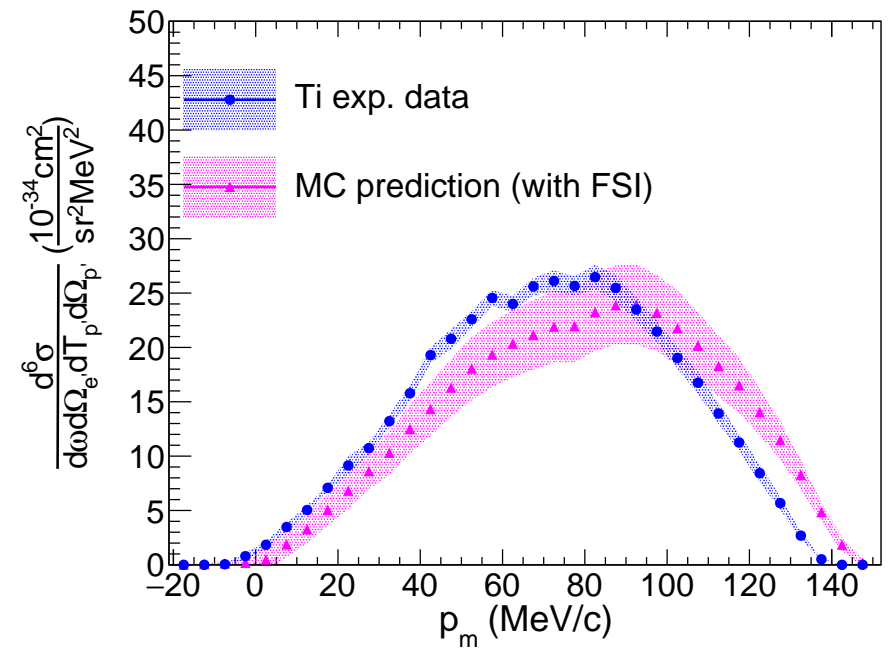

Figure 6.12: Same as Fig. 6.9 but for titanium and integrated over $0<E_{m}<30 \mathrm{MeV}$. 
Chapter 6. Measurement of the $\operatorname{Ar}\left(e, e^{\prime} p\right) X$ and Ti $\left(e, e^{\prime} p\right) X$ cross sections in 134

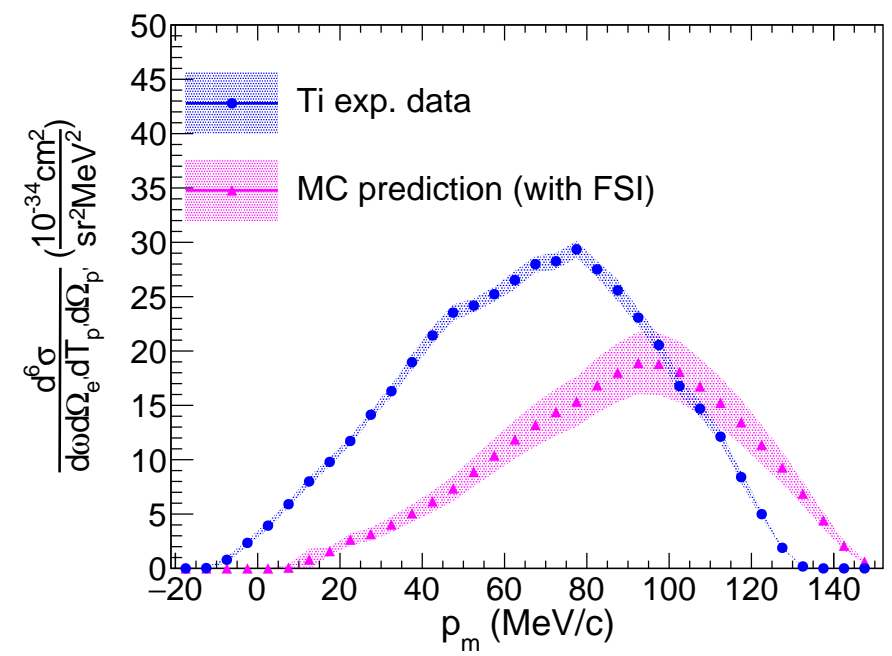

Figure 6.13: Same as Fig. 6.10 but for titanium and integrated over $30<E_{m}<54 \mathrm{MeV}$.

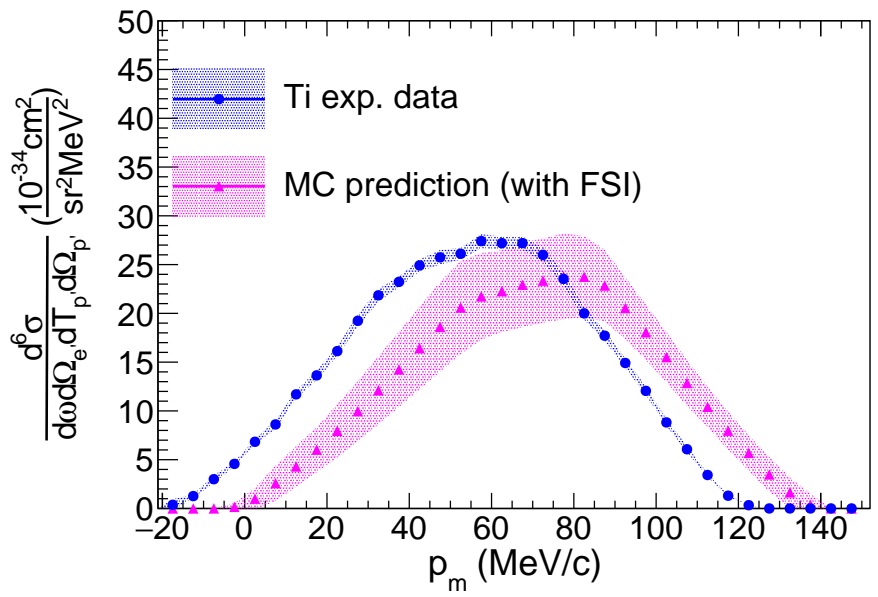

Figure 6.14: Same as Fig. 6.11 but for titanium and integrated over $\quad 54<E_{m}<90 \mathrm{MeV}$. 
not determined from experimental data. Underestimating the widths and the associated overlaps of energy distributions would imply a smaller value for the differential cross section and a shift in the $p_{m}$ distribution between data and MC. We have tested this hypothesis by varying the width of the high-energy states in the test SF and redoing our full analysis, and noticed an improved agreement between data and MC.

More generally, it has to be kept in mind that a clear identification of single particle states in interacting many-body systems - ultimately based on Landau theory of normal Fermi liquids - is only possible in the vicinity of the Fermi surface, corresponding to the lowest value of missing energy, see, e.g., Ref. [172]. An accurate description of the data at large missing energy will require a more realistic model of the nuclear spectral function, taking into account dynamical effects beyond the mean-field approximation, notably nucleon-nucleon correlations, leading to the appearance of protons in continuum states.

\subsection{Summary and Conclusions}

In this paper, we report the first results of the analysis of $\left(e, e^{\prime} p\right)$ data at beam energy $E_{e}=2,222 \mathrm{MeV}$ an electron scattering angle $\theta_{e}=21.5 \mathrm{deg}$, collected in JLab Hall A by the E12-14-012 experiment using Ar and Ti targets. The measured differential cross sections are presented as a function of missing energy and missing momentum, and compared to the predictions of a MC simulation in which the effects of FSI are described within DWIA.

We were able to select coincidence events between the electron and proton spectrometers with high efficiency and low systematic uncertainties. The level of background and systematic uncertainties turned out to be below 4\%, in line with the goals listed in the original JLab E12-14-012 proposal [173]. Overall, the comparison between the data and results of MC simulations, carried out over the lowest missing energy range $0<E_{m}<30 \mathrm{MeV}$ and missing 
Chapter 6. Measurement of the $\operatorname{Ar}\left(e, e^{\prime} p\right) X$ and Ti $\left(e, e^{\prime} p\right) X$ Cross sections in 136 JefFerson LaB Hall A

momentum covered by our measurements appears satisfactory. The larger discrepancies observed at the larger missing energies such as $30<E_{m}<44 \mathrm{MeV}$ re likely to be ascribable to the limitations of the theoretical model based on the mean-field approximation, employed in $\mathrm{MC}$ event generation, which is long known to be inadequate to describe the dynamics of deeply bound nucleons [108]. Understanding these discrepancies at quantitative level will require the inclusion of reaction mechanisms beyond DWIA, such as multi-step processes and multi-nucleon emission triggered by nucleon-nucleon correlations.

The missing energy spectra obtained from our analysis contain valuable new information on the internal structure and dynamics of the nuclear targets, encoded in the positions and widths of the observed peaks.

The determination of these spectra particularly for deep-lying hole excitations is, in fact, a first step towards the derivation of the spectral functions for medium-mass nuclei, such as Ar and $\mathrm{Ti}$, within the framework of LDA, that represents the ultimate aim of our experiment. The Ar and Ti measurements discussed in this article, providing the first $\left(e, e^{\prime} p\right)$ data in the kinematical range relevant to neutrino experiments - most notably DUNE - comprises the first of five datasets collected by the JLab E12-14-012 experiment. The combined analysis of all data, which is currently under way, will provide information of unparalleled value for the development of realistic nuclear models, and will allow the extraction of Ar and Ti spectral functions. 


\section{Chapter 7}

\section{Summary and Conclusions}

This Ph.D. thesis collects a number of published and in-progress results from the E12-14012 experiment at Jefferson Lab, providing new, high-precision data about the $\left(\mathrm{e}, \mathrm{e}^{\prime}\right)$ and $\left(\mathrm{e}, \mathrm{e}^{\prime} \mathrm{p}\right)$ scattering cross sections for argon, titanium, carbon, and aluminum targets. The aim of the E12-14-012 experiment is to determine the spectral function and cross section of argon, which can be used to reduce uncertainty in neutrino oscillation experiments that use argon as the neutrino target. In this Ph.D. thesis we first present the results for the inclusive cross sections, measured at a beam energy of $2.22 \mathrm{GeV}$ and an angle of $15.54^{\circ}$. Chapter 3 describes the measurements of the inclusive cross sections on the carbon and the titanium targets, important also to develop and verify the analysis procedure for the more complicated cryogenic argon gas target. In Chapter 4, we include the first result for the inclusive argon cross section, using the methods developed described in Ch. 3 and adjusted for the extended gas cell target, and in Ch. 5 we present a more detailed paper which collects

all of the inclusive results and make an extensive comparison between them together with a physical interpretation. In all of those measurements we achieved total uncertainties of less than 5\%. Finally, we present in Ch. 6 the first exclusive (e,e'p) cross sections on argon and titanium. A single kinematics is presented in Ch. 6 with electron beam energy $2.22 \mathrm{GeV}$ and an electron scattering angle of $21.5^{\circ}$ and a proton angle of $50.0^{\circ}$, corresponding to a missing momentum of $50 \mathrm{MeV} / \mathrm{c}$. This paper also outlines the procedure that has been developed to determine the effects of final state interactions (FSI) in this experiment. 


\section{Bibliography}

[1] S.L. Glashow, Nucl. Phys. 22, 579 (1961).

[2] S. Weinberg, Phys. Rev. Lett. 19, 1264 (1967).

[3] B. Pontecorvo, Sov. Phys. JETP6, 429 (1958).

[4] Z. Maki, M. Nakagawa, S. Sakata, Prog. Theor. Phys. 28, 870 (1962).

[5] O. Benhar, N. Rocco, Adv. High. Energy. Phys. 2013, 912702 (2013).

[6] V.M. Lobashev, Nucl. Phys. A 719, 153 (2003).

[7] T. Katori, M. Martini, J. Phys. G45, 1 (2016) arXiv:1611.07770 [hep-ph].

[8] F. Capozzi et al., Prog. Part. Nucl. Phys. 102, 48 (2018).

[9] URL https://www.dunescience.org.

[10] B. Abi et al., arXiv preprint arXiv:1807.10334 (2018).

[11] A. Corey et al., (DUNE Collaboration), arXiv preprint arXiv:1307.7335 (2013).

[12] T. de Forest, J.D. Walecka, Adv. Phys. 15, 1 (1966).

[13] J. Gao, Ph.d. thesis, (1999).

[14] J. Mougey et al., Nucl. Phys. A 262, 461 (1976).

[15] J. Alcorn et al., Nucl. Instrum. Meth. A522, 294 (2004). 
[16] Joaquan Lopez Herraiz, Study of the (e,e'p) Quasielastic Reaction in Complex Nuclei: Theory and Experiment. PhD Thesis, Thomas Jefferson National Accelerator Facility, Newport News, VA (United States, (2010).

[17] K. Unser, IEEE Transactions on Nuclear Science,28(3):2344-2346, (1981).

[18] D. Meekins, JLab Drawing TGT-101-1010-0003 (2016).

[19] G. Petratos et al. Jlab experiment e12-10-103, (2010).

[20] Hall A Target Configuration, (2017).

[21] S. N. Santiesteban et al., arXiv:1811.12167 [physics.ins-det].

[22] MO. L. W. and TSAI, Y. S., Rev. Mod. Phys. 41, 205 (1969).

[23] S. R. Dasu, Ph.D. thesis, University of Rochester, 1988.

[24] K. S. Kim, L. E. Wright, Y. Jin, and D. W. Kosik, Phys. Rev. C54, 2515 (1996).

[25] A. Aste, C. von Arx, and D. Trautmann, Eur. Phys. J. A26, 167 (2005).

[26] W. R. Leo, Techniques for nuclear and particle physics experiments (Springer- Verlad Berlin, 1987).

[27] URL https://hallaweb.jlab.org/photos/2015/Hall\%20A/2_011.jpg.

[28] A. Himmel. Talk at FNAL, (2018).

[29] O. Benhar, et al. (The Jefferson Lab E12-14-012 Collaboration), arXiv:1406.4080 [nucl$\mathrm{ex}]$.

[30] W Barry et al. JLab-TN-91-087, (1991). 
[31] WC Barry, JW Heefner, GS Jones, JE Perry, and R Rossmanith. Beam position measurement in the cebaf recirculating linacs by use of pseudorandom pulse sequences. Technical report, (1990).

[32] E Cisbani et al. Nucl. Instrum. Meth. A 496, 305, (2003).

[33] K. G. Fissum et al., Nucl. Instrum. Meth. A 474, 108 (2001). doi:10.1016/S01689002(01)00875-0

[34] Dan Green. High Pt physics at hadron colliders 22. Cambridge University Press, (2005).

[35] Joaquín López Herraiz. Study of the (e, e’p) quasi-elastic reaction in complex nuclei: theory and experiment. Technical report, Thomas Jefferson National Accelerator Facility, Newport News, VA (United States), (2010).

[36] Mo Iodice et al., Nucl. Instrum. Meth. A 411, 108 (1998).

[37] SN. Santiesteban et al., Nucl. Instrum. Meth. A (2001).

[38] K. Unser, IEEE Transactions on Nuclear Science, 28(3):2344-2346, (1981).

[39] Y. Zhihong, Ph.d. thesis. (2013).

[40] O. Benhar, P. Huber, C. Mariani and D. Meloni, Phys. Rept. 700, 1 (2017).

[41] M. Antonello et al. (MicroBooNE, LAr1-ND, and ICARUS-WA104 Collaborations), arXiv:1503.01520 [physics.ins-det].

[42] R. Acciari et al. (DUNE Collaboration), arXiv:1512.06148 [physics.ins-det].

[43] K. Abe et al. (T2K Collaboration), Phys. Rev. D 88, 032002 (2013).

[44] A. M. Ankowski et al., Phys. Rev. D 92, no. 7, 073014 (2015). 
[45] M. Anghinolfi et al., J. Phys. G21, L9 (1995).

[46] O. Benhar, Nucl. Phys. News 26, 15 (2016).

[47] S. Frullani and J. Mougey, Adv. Nucl. Phys. 14, 1 (1984).

[48] A. E. L. Dieperink and P. K. A. de Witt Huberts, Ann. Rev. Nucl. Part. Sci. 40, 239 (1990).

[49] S. Boffi, C. Giusti, F. D. Pacati, Phys. Rept. 226, 1 (1993).

[50] O. Benhar, N. Farina, H. Nakamura, M. Sakuda and R. Seki, Phys. Rev. D 72, 053005 (2005).

[51] O. Benhar, D. Day, I. Sick, Rev. Mod. Phys. 80, 189 (2008).

[52] A. M. Ankowski, O. Benhar, M. Sakuda, Phys. Rev. D 91, 054616 (2015).

[53] N. Rocco, A. Lovato, O. Benhar, Phys. Rev. Lett. 116, 192501 (2016).

[54] O. Benhar and D. Meloni, Nucl. Phys. A 789, 379 (2007).

[55] O. Benhar, P. Coletti, and D. Meloni, Phys. Rev. Lett. 105, 132301 (2010).

[56] O. Benhar and G. Veneziano, Phys. Lett. B 702, 433 (2011).

[57] E. Vagnoni, O. Benhar, and D. Meloni, Phys. Rev. Lett. 118, 142502 (2017).

[58] A. Lovato, S. Gandolfi, J. Carlson, S. C. Pieper and R. Schiavilla, Phys. Rev. Lett. 117, 082501 (2016).

[59] A. Meucci and C. Giusti, Phys. Rev. D89, 117301 (2014).

[60] O. Lalakulich, U. Mosel and K. Gallmeister, Phys. Rev. C86, 054606 (2012). 
[61] J. Nieves, F. Sanchez, I. Ruiz Simo and M. J. Vicente Vacas, Phys. Rev. D85, 113008 (2012).

[62] M. Martini, M. Ericson, G. Chanfray and J. Marteau, Phys. Rev. C81, 045502 (2010).

[63] V. Pandey, N. Jachowicz, T. Van Cuyck, J. Ryckebusch and M. Martini, Phys. Rev. C92, 024606 (2015).

[64] G. D. Megias, J. E. Amaro, M. B. Barbaro, J. A. Caballero, T. W. Donnelly and I. Ruiz Simo, Phys. Rev. D94, 093004 (2016).

[65] J. Heisenberg, J. S. Mccarthy and I. Sick, Nucl. Phys. A164, 353 (1971).

[66] A. M. Selig, C. De Vries, P. K. A. De Witt Huberts and I. E. Zacharov, Nucl. Phys. A476, 413 (1988).

[67] E. F. Romberg, N. S. Wall, D. Blum, J. W. Lightbody and S. Penner, Nucl. Phys. A173, $124(1971)$.

[68] J. Arrington et al., Phys. Rev. Lett. 82, 2056 (1999).

[69] Hall C Collaboration, paper in preparation

[70] A. Aste, C. von Arx, and D. Trautmann, Eur. Phys. J. A26, 167 (2005).

[71] O. Benhar, D. B. Day, and I. Sick, arXiv:0603032 [nucl-ex]. http://faculty •virginia. edu/qes-archive/index.html

[72] I. Sick, D. B. Day, and J. S McCarthy, Phys. Rev. Lett. 45 , 871 (1980).

[73] D. B. Day, J. S. McCarthy, T. W. Donnelly and I. Sick, Ann. Rev. Nucl. Part. Sci. 40, $357(1990)$.

[74] J. S. O'Connell et al., Phys. Rev. C 35, 1063 (1987). 
[75] R. M. Sealock et al, Phys. Rev. Lett. 62 (1989) 1350.

[76] D. B. Day et al., Phys. Rev. C 48, 1849 (1993).

[77] V. Tvaskis, PhD Thesis, Vrije Universiteit, Amsterdam, 2004.

[78] K. Abe et al. (T2K Collaboration), Nucl. Instrum. Meth, A659, 106 (2011).

[79] P. Adamson et al. (NOvA Collaboration), Phys. Rev. Lett. 118, no. 23, 231801 (2017).

[80] L. Ren et al. (MINERvA Collaboration), Phys. Rev. D 95, 072009 (2017).

[81] O. Benhar, A. Fabrocini, S. Fantoni, G. A. Miller, V. R. Pandharipande, and I. Sick, Phys. Rev. C 44, 2328 (1991).

[82] O. Benhar and F. Coppini, paper in preparation.

[83] T.W. Donnelly and I. Sick,, Phys. Rev. C 60, 065502 (1999).

[84] E.J. Moniz et al, Phys. Rev. Lett. 26, 445 (1971).

[85] B. Abi et al. (DUNE Collaboration), arXiv:1807.10334 [physics.ins-det].

[86] A. A. Aguilar-Arevalo et al. (MiniBooNE Collaboration), arXiv:1805.12028 [hep-ex].

[87] A. M. Ankowski, P. Coloma, P. Huber, C. Mariani, and E. Vagnoni, Phys. Rev. D 92, $091301(2015)$.

[88] K. Abe et al. (T2K Collaboration), Phys. Rev. Lett. 118, 151801 (2017).

[89] M. .A. Acero (NovA Collaboration), Phys. Rev. D 98, 032012 (2018).

[90] L. Alvarez-Ruso et al., Prog. Part. Nucl. Phys. 100, 1 (2018).

[91] A. M. Ankowski, and C. Mariani, J. Phys. G 44, 054001 (2017). 
[92] H. Dai, et al., (The Jefferson Lab Hall A Collaboration), Phys. Rev. C98, 014617 (2018).

[93] In 2016, the Quadrupole magnet closest to the target (Q1) was replaced with a normal conducting quad, with similar magnetic properties.

[94] A. Meucci, F. Capuzzi, C. Giusti and F. D. Pacati, Phys. Rev. C 67, 054601 (2003).

[95] T. de Forest, Nucl. Phys. A392, 232 (1983).

[96] S. Boffi, C. Giusti, F. D. Pacati, and M. Radici, Electromagnetic Response of Atomic Nuclei (Clarendon, Oxford, UK, 1996).

[97] P. K. A. de Witt Huberts, J. Phys. G 16, 507 (1990).

[98] D. Rohe et al., Phys. Rev. Lett. 93, 182501 (2004).

[99] K. Abe et al. (T2K Collaboration), Phys. Rev. Lett. 121, 171802 (2018).

[100] K. Abe et al. (Hyper-Kamiokande Proto-Collaboration), Prog. Theor. Exp. Phys. 2015, $053 \mathrm{C} 02$ (2015).

[101] H. Dai et al. (Jefferson Lab Hall A Collaboration), Phys. Rev. C 99, 054608 (2019).

[102] J. K. Tuli, Nuclear Wallet Cards, https://www.nndc.bnl.gov/wallet, 2011.

[103] J. S. O’Connell et al., Phys. Rev. Lett. 53, 1627 (1984).

[104] D. T. Baran et al., Phys. Rev. Lett. 61, 400 (1988).

[105] A. N. Antonov and I. Zh. Petkov, Nuovo Cimento A 94, 68 (1986).

[106] D. B. Day et al., Phys. Rev. C 40, 1011 (1989).

[107] R. L. Talaga, J. J. Grudzinski, S. Phan-Budd, A. Pla-Dalmau, J. E. Fagan, C. Grozis, and K. M. Kephart, Nucl. Instrum. Meth. A 861, 77 (2017). 
[108] O. Benhar, Nucl. Phys. News. 26, 15 (2018).

[109] M. Riou et al., Rev. Mod. Phys. 37, 375 (1965).

[110] G. Jacob and Th. A. J. Maris, Nucl. Phys. A31, 139 (1962).

[111] D. H. E. Gross and R. Lipperheide, Nucl. Phys. A150, 449 (1960).

[112] S. Boffi, C. Giusti, F. Pacati, and S. Frullani, Nucl. Phys. A 319, 461 (1979).

[113] C. Giusti and F. Pacati, Nucl. Phys. A 485, 461 (1988).

[114] S. Frullani and J. Mougey, Adv. Nucl. Phys. 14, 1 (1984).

[115] A.E.L. Dieperink and P.K.A. de Witt Huberts, Ann. Rev. Nucl. Part. Sci. 40, 239 (1990).

[116] O. Benhar and V.R. Pandharipande, Phys. Rev. C 47, 2218 (1993).

[117] O. Benhar, A. Fabrocini, S. Fantoni, and I. Sick, Nucl. Phys. A 579, 493 (1994).

[118] A. Ankowski, O. Benhar, and M. Sakuda, Phys. Rev. D 91, 033005 (2015)

[119] O. Benhar, P. Huber, C. Mariani, and D. Meloni, Phys. Rep. 700, 1 (2017).

[120] O. Benhar, N. Farina, H. Nakamura, M. Sakuda, and R. Seki, Phys. Rev. D 72, 053005 (2005).

[121] O. Benhar and D. Meloni, Phys. Rev. D 80, 073003 (2009).

[122] C.-M. Jen, A. M. Ankowski, O. Benhar, A. P. Furmanski, L. N. Kalousis, and C. Mariani, Phys. Rev. D 90, 093004 (2014).

[123] O. Benhar and D. Meloni, Nucl. Phys. A 789, 379 (2007). 
[124] A. M. Ankowski, O. Benhar, and N. Farina, Phys. Rev. D 82, 013002 (2010).

[125] O. Benhar, O. Coletti, and Davide Meloni, Phys. Rev. Lett. 105, 132301 (2010).

[126] A. M. Ankowski, O. Benhar, T. Mori, R. Yamaguchi, and M. Sakuda, Phys. Rev. Lett. 108, 052505 (2012).

[127] A. M. Ankowski, Phys. Rev. C 86, 024616 (2012).

[128] A. M. Ankowski and O. Benhar, Phys. Rev. D 88, 093004 (2013).

[129] A. M. Ankowski, Phys. Rev. D 92, 013007 (2015).

[130] A. M. Ankowski, M. B. Barbaro, O. Benhar, J. A. Caballero, C. Giusti, R. GonzálezJiménez, G. D. Megias, and A. Meucci, Phys. Rev. C 92,

[131] E. Vagnoni, O. Benhar, and D. Meloni, Phys. Rev. Lett. 118, 142502 (2017).

[132] A. M. Ankowski, Phys. Rev. C 96, 035501 (2017).

[133] S. Fukuda et al., Nucl. Instrum. Methods Phys. Res. A 599, 28 (2003).

[134] A. A. Aguilar-Arevalo et al. (The MiniBooNE Collaboration), Nucl. Instrum. Methods Phys. Res A 501, 418 (2009).

[135] B. Abi et al. (DUNE Collaboration), J. Instr. 15, T08008 (2020).

[136] C. Barbieri, N. Rocco, and V. Somà, Phys. Rev. C 100, 062501 (2019).

[137] H. Dai, et al. (Jefferson Lab Hall A Collaboration), Phys. Rev. C 98, 014617 (2018).

[138] M. Murphy, et al. (Jefferson Lab Hall A Collaboration), Phys. Rev. C 100, no.5, 054606 (2019).

[139] SIMC, https://hallcweb.jlab.org/wiki/index.php/SIMC_Monte_Carlo. 
[140] T. de Forest, Nucl. Phys. A392, 232 (1983).

[141] Frankfurt, M. Strikman, M. Zhalov, Physics Letters B, 503, 73, 2001

[142] A. E. L. Diepereink, T. de Forest Jr., I. Sick, and M.A. Brandenburg, Phys. Lett. B 63, (1976).

[143] I. Sick, D. B. Day, and J. S. McCarthy, Phys. Rev. Lett. 45, 871 (1980).

[144] T. W. Donnelly, and I. Sick, Phys. Rev. C 60, 065502 (1999).

[145] S. Boffi, C. Giusti, F. D. Pacati, Phys. Rep. 226, 1 (1993).

[146] S. Boffi, C. Giusti, F. Pacati, M. Radici, Electromagnetic response of atomic nuclei, Clarendon, Oxford, 1996.

[147] S. Boffi, F. Cannata, F. Capuzzi, C. Giusti, F. D. Pacati, Nucl. Phys. A379, 509 (1982).

[148] M. Radici, S. Boffi, S. C. Pieper, and V. R. Pandharipande, Phys. Rev. C 50, 3010 (1994).

[149] C. Bisconti, F. Arias de Saavedra, and G. Cò, Phys. Rev. C 75, 054302 (2007).

[150] C. Giusti, F. D. Pacati, Nucl. Phys. A473, 717 (1987).

[151] C. Giusti, A. Meucci, F. D. Pacati, G. Co', V. De Donno, Phys. Rev. C 84, 024615 (2011).

[152] J. M. Udías, P. Sarriguren, E. Moya de Guerra, J. A. Caballero, Phys. Rev. C 48, 2731 (1993).

[153] A. Meucci, C. Giusti, F. D. Pacati, Phys. Rev. C 64, 014604. (2001). 
[154] M. Bernheim, A. Bussière, J. Mougey, D. Royer, D. Tarnowski, S. Turck-Chièze, S. Frullani, S. Boffi, C. Giusti, F. D. Pacati, G. P. Capitani, E. de Sanctis, G. J. Wagner, Nucl. Phys. A375, 381 (1982).

[155] S. Boffi, C. Giusti, F. D. Pacati, Nucl. Phys. A336, 437 (1980).

[156] J. R. Vignote, M. C. Martínez, J. A. Caballero, E. Moya de Guerra, J. M. Udías, Phys. Rev. C 70, 044608 (2004).

[157] P. Finelli, N. Kaiser, D. Vretenar, and W. Weise, Nucl. Phys. A 735, 449 (2004).

[158] P. Finelli, N. Kaiser, D. Vretenar, and W. Weise, Nucl. Phys. A 770, 1 (2006).

[159] T. Nikšić, N. Paar, D. Vretenar, and P. Ring, Comput. Phys. Commun. 185, 1808 (2014).

[160] Meng Wang, G. Audi, F. G. Kondev, W.J. Huang, S. Naimi, and Xing Xu, Chin. Phys. C 41, 030003 (2017).

[161] J. Chen, Nucl. Data Sheets 149, 1 (2018).

[162] T.W. Burrows, Nucl. Data Sheets 108, 923 (2007).

[163] G. Mairle, M. Seeger, H. Reinhardt, T. Kihm, K.T. Knöpfle, and Chen Lin Wen, Nucl. Phys. A565, 543 (1993).

[164] P. Doll, G. Mairle, H. Breuer, K.T. Knöpfle, T. Tohei, and G.J. Wagner, J. Phys. G 5, $1421(1979)$.

[165] E. K. Warburton and J.A. Becker, Phys. Rev. C 40, 2823 (1989).

[166] P. Doll, Nucl. Phys. A292, 165 (1977).

[167] L.W. Whitlow, Ph.D. Thesis, Stanford University, 1990, SLAC-REPORT-357 (1990). 
[168] A. Aste, C. von Arx, and D. Trautmann, Eur. Phys. J. A26, 167 (2005).

[169] E. D. Cooper, S. Hama, B. C. Clark, Phys. Rev. C 80, 034605 (2009).

[170] E. D. Cooper, S. Hama, B. C. Clark, R. L. Mercer, Phys. Rev. C 47, 297 (1993).

[171] Blanpied, G.S. and Ritchie, B.G. and Barlett, M. L. and Fergerson, R. W. and McGill, J.A. and Wildenthal, B.H., Phys. Rev. C 37, 1304-1306 (1988)

[172] O. Benhar and S. Fantoni, Nuclear Matter Theory (Taylor \& Francis, Oxford, 2020).

[173] O. Benhar et al., arXiv:1406.4080. 